\title{
Ceramics Division \\ Fy 2004 Programs and Accomplishments
}
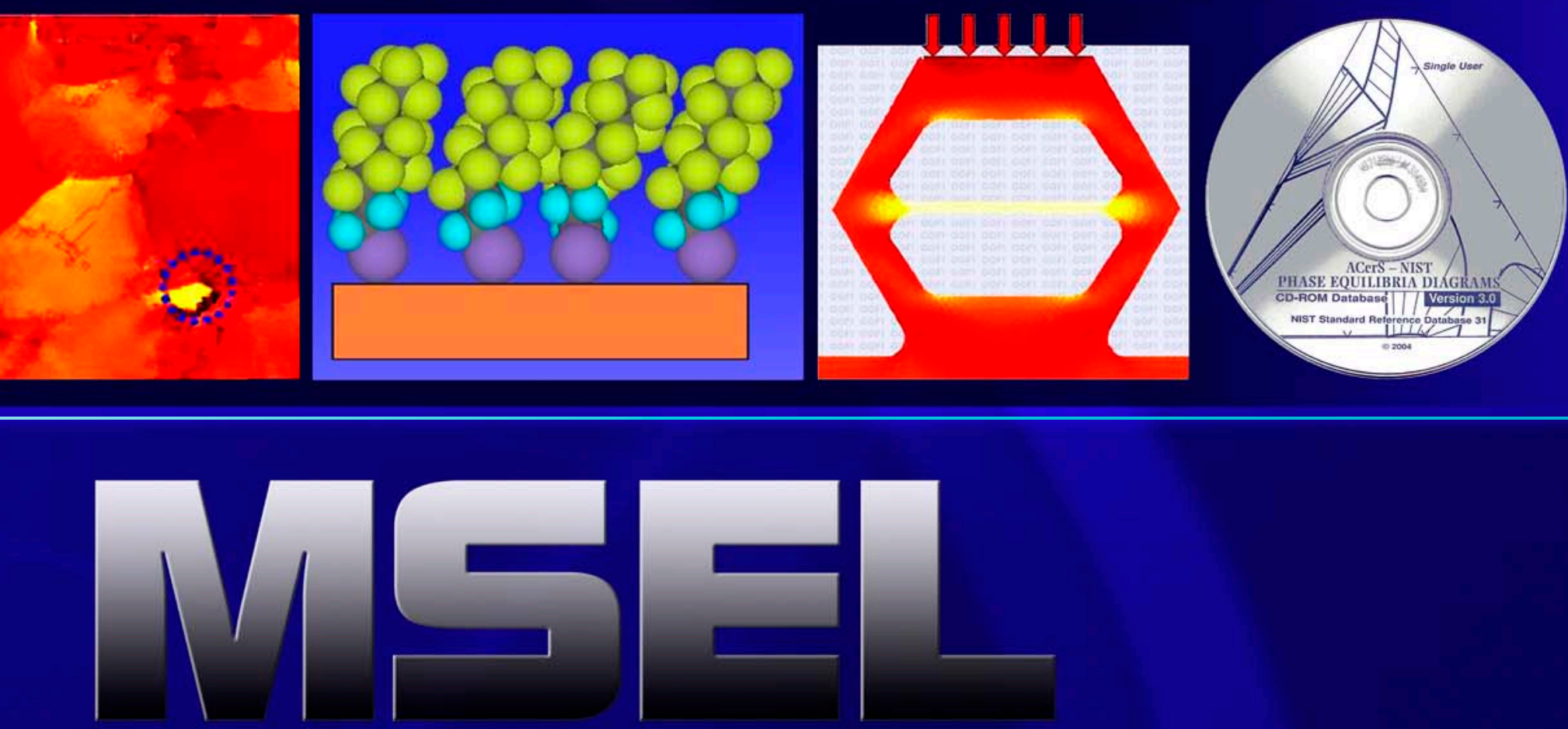

Materials Science and Engineering Laboratory

\section{NGT}

\section{National Institute of}

Standards and Technology

Technology Administration

U.S. Department of Commerce

NISTIR 7124

September 2004 


\section{On the Cover:}

On the front cover, from right to left, and continuing onto the back cover, the images shown are, respectively:

Fig. 1 - The ACerS-NIST Phase Equilibria Diagrams CDROM, the latest achievement in the longstanding NIST collaboration with the American Ceramic Society. (See also the highlight, "Data and Standards Resources.")

Fig. 2 - Model of compressively loaded hexagonal theta test specimen with a well defined tensile gage section. (See also the project report, "Mechanical Metrology for Small-Scale Structures.”)

Fig. 3 - Schematic illustration of the formation of molecular gradients on elastomeric substrates via self-assembly. (See also the highlight, "Mapping Surface Chemistry and Molecular Orientation with Combinatorial Near Edge X-ray Absorption Fine Structure.”)

Fig. 4 - Piezoelectric response of a polycrystalline thin film predicted using twodimensional object oriented finite element modeling (OOF). The strain normal to the surface is depicted for an applied bias of $1 \mathrm{~V}$ across a $100 \mathrm{~nm}$ thick film using a $100 \mathrm{~nm}$ diameter electrode, where the electrode is positioned across multiple grains of differing orientations. (See also the project report, "Experimental and Theoretical Influence of Thin Film Texture on Ferroelectric Hysteresis.”)

Fig. 5 - Schematic of the cubic fluorite structure pertinent to YOF, an intermediate phase found in the phase equilibrium study of superconducting $\mathrm{Ba}_{2} \mathrm{YCu}_{3} \mathrm{O}_{7}$ by the " $\mathrm{BaF} \mathrm{F}_{2}$ ex-situ" process. (See also the project report, "Phase Relations of High $\mathrm{T}_{\mathrm{C}}$ Superconductors.") 
National Institute of

Standards and Technology

Arden L. Bement, Jr.

Director

Technology

Administration

Phillip J. Bond

Undersecretary of

Commerce for Technology

U.S. Department

of Commerce

Donald L. Evans

Secretary

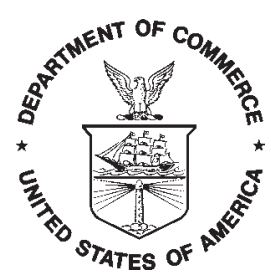

\section{Materials Science and Engineering Laboratory}

\section{FY 2004 Programs and ACcOMplishments}

\section{Ceramics Division}

Debra L. Kaiser, Chief

Ronald G. Munro, Deputy 
Certain commercial entities, equipment, or materials may be identified in this document in order to describe an experimental procedure or concept adequately. Such identification is not intended to imply recommendation or endorsement by the National Institute of Standards and Technology, nor is it intended to imply that the entities, materials, or equipment are necessarily the best available for the purpose. 


\section{Table of Contents}

Executive Summary 1

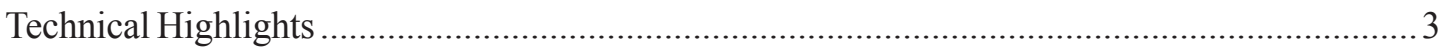

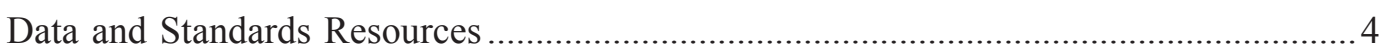

Advanced Measurement Capabilities ............................................................................ 7

Outreach for Advanced Technology Dissemination .................................................... 10

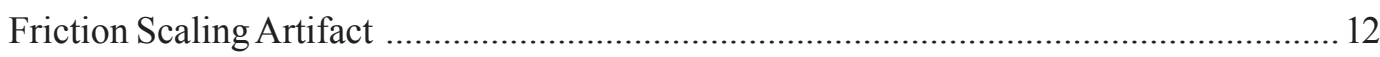

First-Principles Studies of Technologically Important

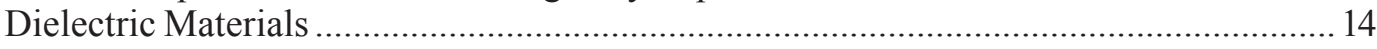

Anomalous Elastic Behavior for $\mathrm{SrTiO}_{3}$ Thin Films

Grown on Si(001) Observed by X-ray Diffraction ..................................................... 16

Mapping Surface Chemistry and Molecular Orientation with

Combinatorial Near-Edge X-ray Absorption Fine Structure ......................................... 18

Advances in X-ray Powder Diffraction Quantitative

Analysis SRMs

Advanced Manufacturing Processes .22

Synchrotron X-ray Measurements in Support of Solid Oxide Fuel Cell Development .23

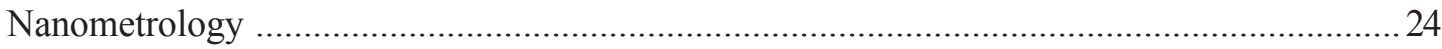

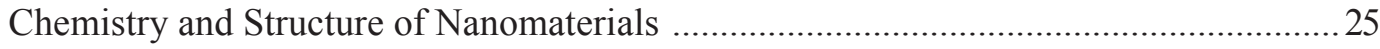

Thermochemistry and Metrology of Interfacial Interactions …......................................26

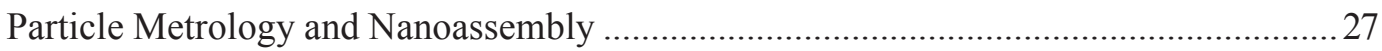

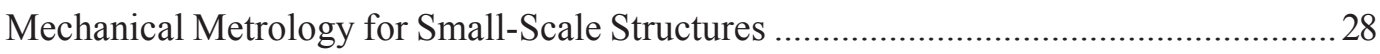

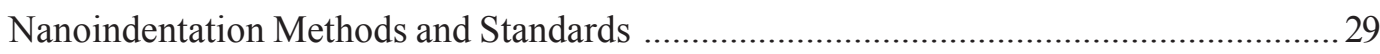

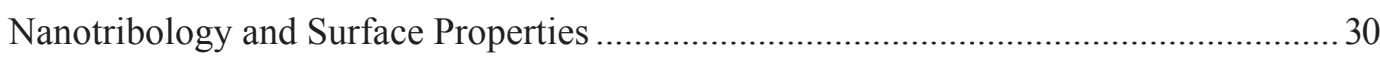

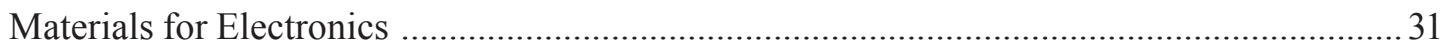

Combinatorial Tools for Materials Science ……................................................................ 32

Spectroscopy, Diffraction, and Imaging of Electronic Materials ........................................33

Phase Equilibria and Properties of Dielectric Ceramics ....................................................... 34 


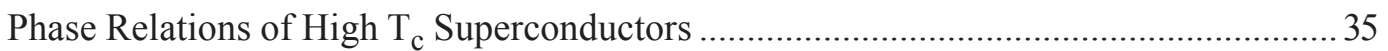

Metrology and Standards for Optoelectronic Materials ................................................... 36

Experimental and Theoretical Influence

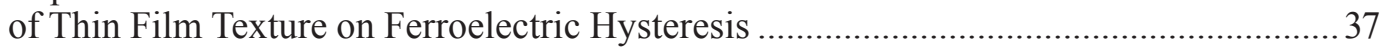

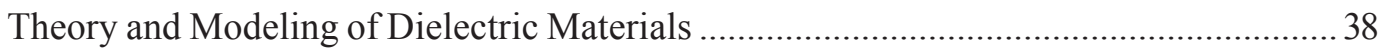

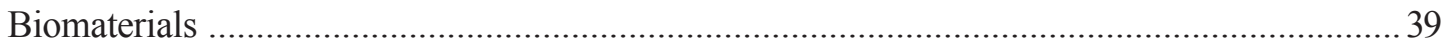

Controlled Size and Shape UHMWPE Particle SRM

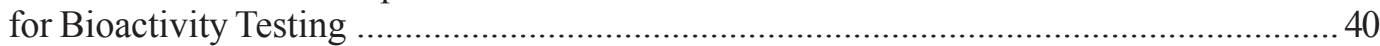

Data, Reference Materials, and Measurement Methods ....................................................... 41

Ceramics Division FY04 Annual Report Publication List ...................................................... 45

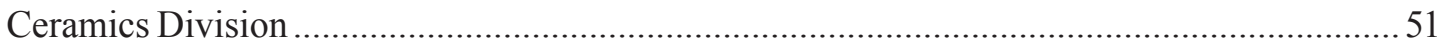

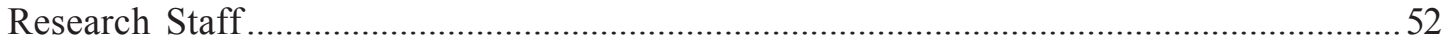

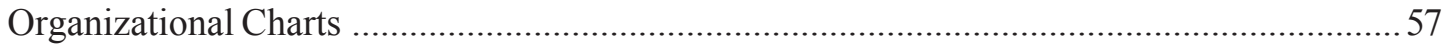




\section{Executive Summary}

It has been a challenging yet productive year for the Ceramics Division. Faced with an unexpected, substantial budget cut, the division had to reassess programs, re-evaluate priorities for personnel and projects, and curtail and modify research plans. In the midst of this uncertain and trying time, the excellence and professionalism of our staff was easy to discern. Through ingenuity, adaptation, and perseverance, hardpressed efforts often led to notable accomplishments.

In accord with the mission of NIST, the Ceramics Division has maintained its balanced portfolio of measurements, standards and data activities. Many of these activities have been refocused to Nanometrology and Materials for Electronics, two of the five program areas in the Materials Science and Engineering Laboratory (MSEL). The division also contributed to Biomaterials and Advanced Manufacturing Processes, two other MSEL program areas. Experimental, theoretical and modeling approaches have been employed to accomplish the advances described for each program area.

It was a remarkable year for technology transfer, led by our world class efforts in evaluated, scientific databases. The Ceramics WebBook, providing data online since 1997, remained the eleventh most visited site at NIST. The renowned FIZ-NIST Inorganic Crystal Structure Database issued two updates in one year, and the longstanding ACerS-NIST Phase Equilibria Diagrams project achieved new heights this year with the release of a brand new, comprehensive CDROM database product. These staples of core mission efforts were crowned by our outreach activities that provided critical mission support to federal agencies, CRADAs, and consortia, including special contributions to the U.S. Customs and Border Protection Directorate in the Department of Homeland Security, NAVSEA in Defense, and the U.S. Mint. These efforts and more are described in our Highlights.

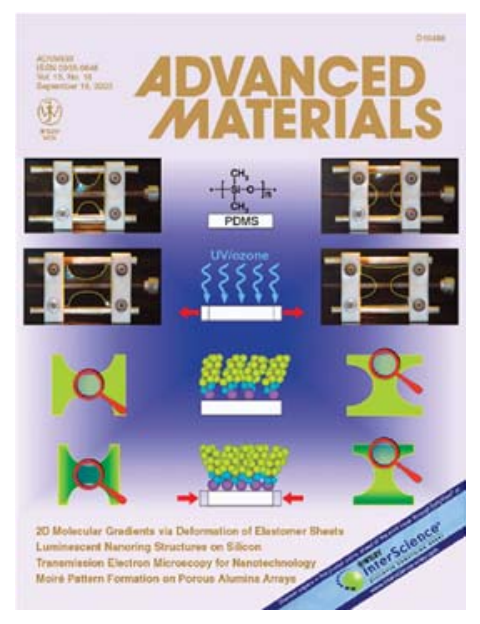

Figure 1: Synchrotron materials research contributed a cover story for the journal Advanced Materials.
It was also a year of new beginnings. With considerable celebration, NIST inaugurated its new Advanced Measurement Laboratory (AML) featuring extraordinary environmental controls. We are extremely pleased to represent MSEL in this new facility with two advanced capabilities: one devoted to nanotribology, and the other to high resolution x-ray metrology. Both of these new facilities, as well as our unique synchrotron radiation facilities at the National Synchrotron Light Source and the Advanced Photon Source, are described in our Highlight on Advanced Measurement Capabilities.

Figure 2: Dr. Edwin R. Fuller, Jr. was presented the American Ceramic Society's Robert D. Sosman Award for his outstanding achievement in basic ceramic science.

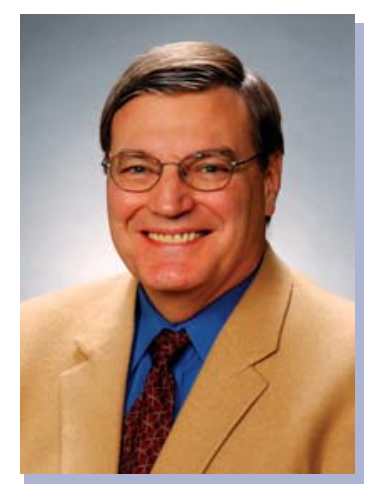

Several other particularly notable scientific achievements are featured in our Highlights. We have applied our novel nondestructive synchrotron-based analytical tool for mapping bond chemistry and molecular orientation to study in situ the creation of self-assembled monolayer gradients. Our synchrotron facilities were key to revealing fundamental aspects of epitaxial growth of $\mathrm{SrTiO}_{3}$ thin films on $\mathrm{Si}$, candidate materials for next-generation Si transistor technology. First-principles theoretical studies of dielectric materials for electronic and wireless applications have provided insight into the atomic interactions that dictate the properties of these materials. With our advanced nanofriction measurement capabilities in the AML, we were able to identify and quantify an unintended artifact of the measurement procedure that degraded the measurement accuracy. We delivered the first nominally-crystalline standard reference material for quantitative analysis by powder diffraction methods that is certified for amorphous content.

In all of these activities, our commitment to a strong, productive future is clear. These projects and many more, described in this report, are well on their way to forming a foundation for next generation research in nanomaterials and advanced ceramics.

Debra L. Kaiser

Chief, Ceramics Division 



\section{Technical Highlights}

The Technical Highlights section of this Report comprises eight examples of the exciting outputs of the Ceramics Division. They are:

- Data and Standards Resources

- Advanced Measurement Capabilities

- Outreach for Advanced Technology Dissemination

- Friction Scaling Artifact

- First-Principles Studies of Technologically Important Dielectric Materials

- Anomalous Elastic Behavior for $\mathrm{SrTiO}_{3}$ Thin Films Grown on $\mathrm{Si}(001)$ Observed by X-ray Diffraction

- Mapping Surface Chemistry and Molecular Orientation with Combinatorial Near-Edge X-ray Absorption Fine Structure

- Advances in X-ray Powder Diffraction Quantitative Analysis SRMs 


\section{Data and Standards Resources}

Advances in technology and the vitality of basic research have an unequivocal dependence on reliable data. Indeed, the technology transfer between research and application is facilitated and sometimes enabled by the development and accessibility of carefully designed and evaluated databases. The MSEL Ceramics Division has excelled in its leadership in data resources for crystallography, phase equilibria diagrams, and materials property data.

\section{Ronald G. Munro, Terrell A. Vanderah, and Vicky L. Karen}

$T^{\mathrm{s}}$ he issue of reliable data has been examined vigorously in numerous studies over the last three decades. Early assessments, such as Critical Surveys of Data Sources (NBS SP 396-1, 1978), voiced concerns over the availability of data, while other studies, such as Economic Effects of Corrosion (NBS SP 511-1\&2, 1978), noted the influential role that data can perform as a component of a sound economy. More recently, defense and security concerns, Materials Research to Meet 21st Century Defense Needs (NMAB-498, 2002), have continued to stress the need for reliable data.

\begin{tabular}{|c|c|c|c|c|c|}
\hline & & \\
\hline & & $\begin{array}{l}\text { X-Ray } \\
\text { SRMs }\end{array}$ & $\begin{array}{c}\text { Crystal } \\
\text { Data }\end{array}$ & $\begin{array}{l}\text { Materials } \\
\text { Properties }\end{array}$ & $\begin{array}{l}\text { Phase } \\
\text { Equilibria }\end{array}$ \\
\hline \multirow{3}{*}{ Core NIST functions } & Metrology standards & $\bullet$ & & & \\
\hline & Metrology facilities & $\bullet$ & & & \\
\hline & Evaluation methods & & $\bullet$ & $\bullet$ & $\bullet$ \\
\hline Strategic focus areas & Information management & & $\bullet$ & $\bullet$ & $\bullet$ \\
\hline \multirow{2}{*}{ National needs } & Materials data & & $\bullet$ & 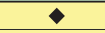 & $\bullet$ \\
\hline & Measurement tracebility & $\bullet$ & & & \\
\hline NIST obligation & Standard reference data & & $\bullet$ & 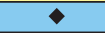 & $\bullet$ \\
\hline
\end{tabular}

Figure 1: Programmatic contributions of the Data and Standards Technology Group to the NIST mission.

The Ceramics Division has made a substantial effort to address the multiple needs for materials data, the scientific fundamentals for data evaluation methodology, and the technological issues involved in optimizing the utility of the data resources. Programmatically, Figure 1, the data and standards technology projects are well attuned to NIST's core mission and its strategic focus efforts, and there is abundant evidence that our objectives are being met with considerable success.

There are several technology transfer mechanisms that can be used for data and standards work, including traditional publications, internet distribution, software products for personal computers, and standard reference materials. The Division's most readily visible mechanism for data technology transfer is provided by the Ceramics WebBook that is featured on the Division's internet

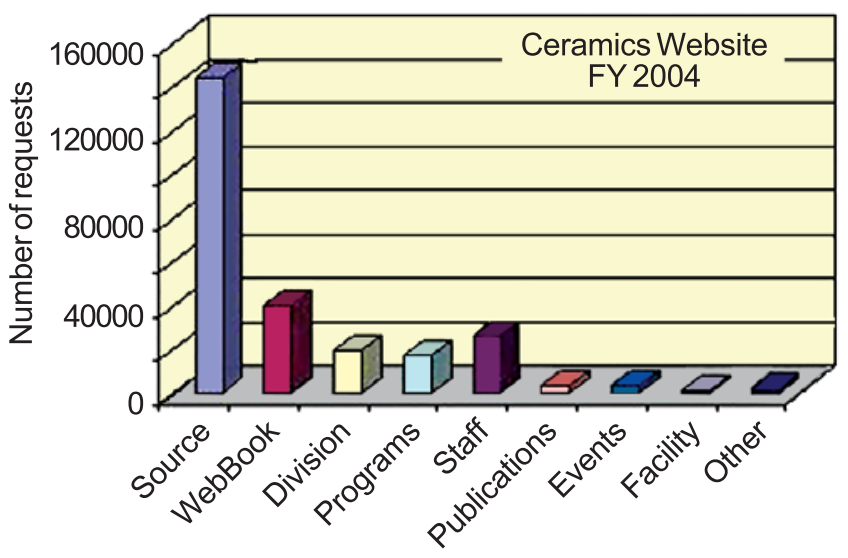

Figure 2: Activity seen by the Ceramics Division's homepage, showing the substantial attraction to property data.

website. This site provides evaluated data for the physical, mechanical, and thermal properties of advanced ceramics, particularly structural ceramics (NIST Standard Reference Database Number 30) and high-temperature superconductors (NIST Standard Reference Database Number 62). The activity on this site, Figure 2, is a clear indicator of both the strong interest and the persistent need for materials data. The site attracts more than 11,000 page requests per month of which approximately $78 \%$ are data downloads (nearly 9000 data tables).

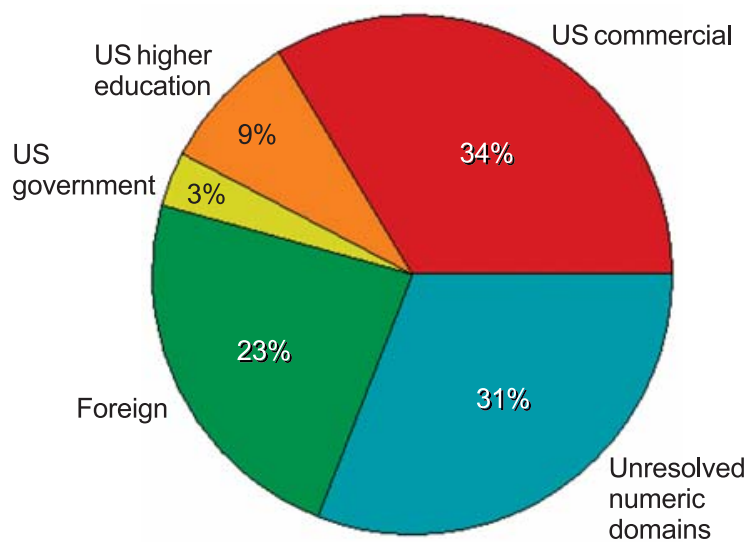

Figure 3: The relative distribution of users downloading data from the Ceramics WebBook.

The distribution of users, Figure 3, shows that all sectors of the ceramics community have a keen interest in the evaluated data provided by NIST.

Another very important mechanism for technology transfer is provided by the Standard Reference Data Act (Public Law 90-3960, 1968). Under the SRD Act, NIST can pursue technology transfer through partnerships that broadly serve the public interest. These partnerships 
typically involve costs in excess of our Congressionally funded base operations and consequently result in small charges for the data products. Two prominent collaborations of this type between the Ceramics Division and private sector nonprofit organizations have brought considerable credit and recognition to NIST.
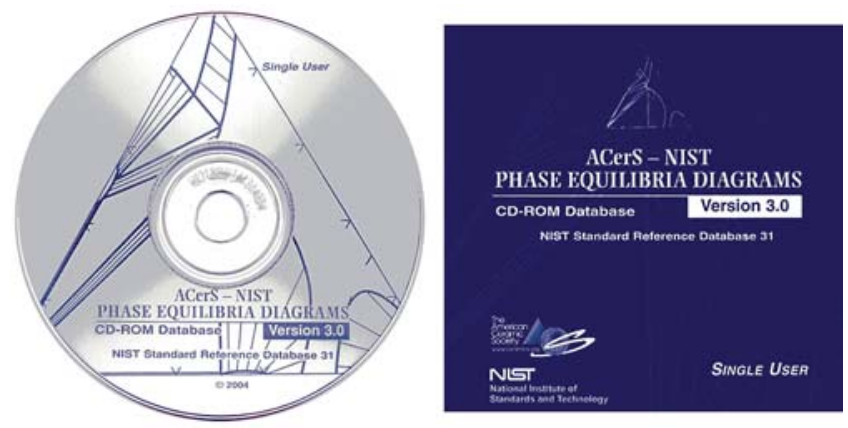

Figure 4: Phase Equilibria Diagrams CD-ROM Database Version 3.0 (NIST Standard Reference Database Number 31), released in March 2004, provides comprehensive coverage of all diagrams and commentaries published to date in the twenty printed volumes of the series.

The longstanding NIST collaboration with the American Ceramic Society (ACerS) on phase diagrams provides a clear example of the remarkable productivity that can result from these partnerships. Now led by Dr. Terrell Vanderah, the ACerS-NIST collaboration achieved one of its most anticipated goals this year, Figure 4, the development of a new computerized version of NIST Standard Reference Database Number 31, Phase Equilibria Diagrams.

Phase equilibria diagrams are used throughout the ceramics industry to understand and control the complex phenomena which increasingly underlie advanced processing and materials performance. These diagrams provide maps of the equilibrium chemical and structural behaviors exhibited by materials and provide critical starting information for the rational design of materials processing schemes, for quality assurance efforts, and for the optimization of the physical and chemical properties of advanced materials.

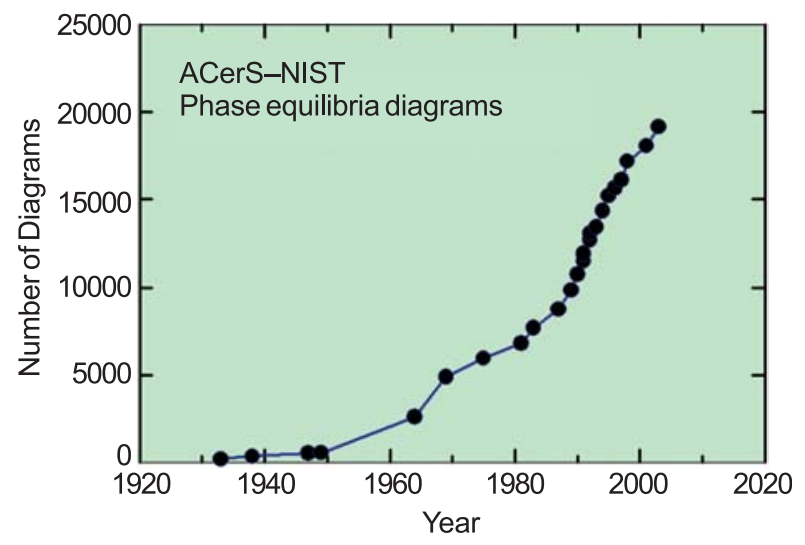

Figure 5: Evolution of the compilation of phase diagrams in the ACerS-NIST collaborative database.
The ACerS-NIST database began with the publication of 178 diagrams in 1933. Since then, the collection has evolved, Figure 5, to contain nearly 20,000 diagrams. Originally, the diagrams were distributed in printed volumes, now amounting to thirteen regular book volumes, four topical volumes, and three annual volumes that serve as supplements to the regular volumes.

Today, both printed and computerized versions are provided and are made available through ACerS.

The newest addition to the series, Figure 6, is the special topical volume, Phase Diagrams for Electronic Ceramics I, edited by Robert S. Roth. This collection concentrates on the technically important oxides of titanium, niobium, and tantalum. Additionally, a newly updated Cumulative Index 2003 was published this year providing comprehensive coverage of all the ACerS-NIST content published to date. All of the new diagrams and commentaries are included in the CD-ROM database.
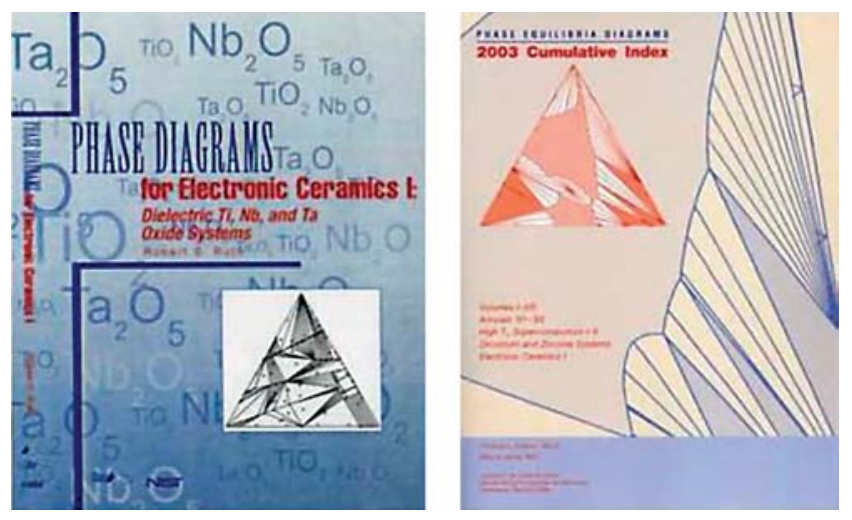

Figure 6: Recent ACerS-NIST printed data products include a topical volume on electronic ceramics and an updated cumulative index for all content published to date.

The mutual commitment of ACerS and NIST to this collaboration was reaffirmed in May of this year with the signing of a new five-year agreement.

The ability to develop partnerships in the public interest also has enabled a successful collaboration, led by Dr. Vicky Karen, between NIST and Fachinformationszentrum Karlsruhe Gesellschaft fur wissenshaftlich-techmische Information $\mathrm{mbH}$ (FIZ). This collaboration, Figure 7, has resulted in the development of NIST Standard Reference Database Number 84, the comprehensive FIZ-NIST Inorganic Crystal Structure Database.

Crystallographic information is critical to efforts to visualize, explain, and predict behaviors of chemicals and materials, as well as to establish the identity of materials in forensic investigations. Literally tens of thousands of structures have been reported. Those reports, however, have varying degrees of reliability and completeness. Consequently, researchers and instrument manufacturers have a 
critical need for evaluated data and the mathematical tools capable of distinguishing good structures from bad structures and compatible data from incompatible data. Through NIST's efforts, the use of standard crystallographic cells, unique reduced cells, and the powerful mathematical matrix methods for the application of group theory have become fundamental to the analysis and identification of crystal structures.

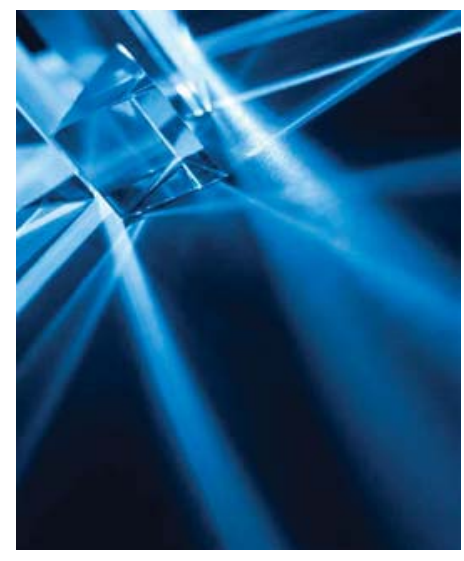

Figure 7: The FIZ-NIST Inorganic Crystal Structure Database contains approximately 70,000 entries providing full structural, three-dimensional crystallographic parameters.

The FIZ-NIST collaboration provides a comprehensive compilation of three-dimensional crystallographic data. The components of a typical entry include data on the conventional cell, the reduced cell, lattice type, space group, lattice parameters, atomic coordinates, d-spacings, chemical name, chemical formula, material class indicators, and literature reference citations.

The collaboration is built on shared and complementary responsibilities. NIST is responsible for the essential scientific and informatic aspects of the work, while FIZ provides data abstracting and related interactions with the International Union of Crystallography, particularly for bibliographic information. Data evaluation is done jointly.

The latest version of the database, released this year, contains more than 70,000 critically evaluated data entries. The database is licensed to research facilities, instrument manufacturers, educational institutions, and individuals. Distributor license agreements with instrument manufacturers are propagated subsequently as user licenses to the instrument buyers. As a result, these data are used every day in defense, aerospace, electronic, petroleum, chemical, and homeland defense related industries, as well as in government and academic research facilities worldwide.

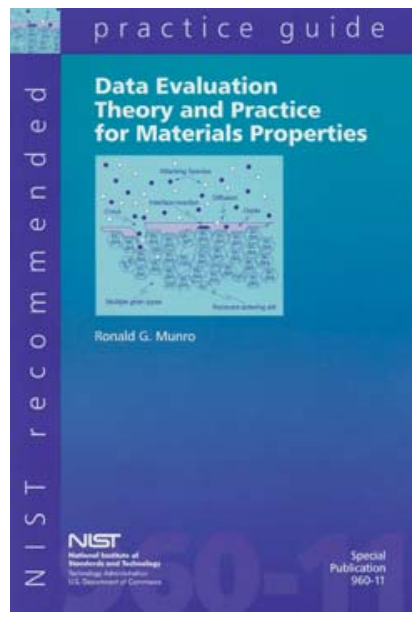

Figure 8: NIST Recommended Practice Guide, SP 960-11, examines pivotal issues in data evaluation.

Additional technology transfer has been achieved through the distribution of NIST Recommended Practice Guide, SP 960-11, Data Evaluation Theory and Practice for Materials Properties, Figure 8.

This Guide presents a unique view of data evaluation as a scientific discipline. A theoretical foundation is developed from the formal underpinnings of materials metrology, and an extensive collection of examples is used to examine the issues of accessibility, reproducibility, consistency, and predictability with distinctions being made among definitive relations, correlations, derived and semiempirical relations, heuristic theories, and value estimates. Critical discussions include the use of properties as parameters in models, the interpretation of ad hoc parameters, and the treatments of procedural properties, response dependent properties, and system dependent data.

Completed in FY2003 and printed in June 2003, more than 1200 hard copies have been issued to interested individuals, and an electronic version in pdf format, available since January 2004, has resulted in the distribution of another 1600 copies.

For a greater appreciation of the full range of data and standards activities in the Ceramics Division, see the tabulation of our products in the section, Databases and Standards, later in this report.

\section{For More Information on this Topic}

P. Schenck, J. Harris, C. Sturrock (Ceramics Division, NIST); A. Belsky (SRD, NIST Technology Services); R. Roth, J. Hastie, M. Harne, A. Mighell (Research Associates, Ceramics Division, NIST); D. Watson (Cambridge Crystallographic DB); M. Mecklenborg, J. You, C. Cedeno, E. Farabaugh, K. Hill, N. Swanson, X.M. Wang (American Ceramic Society); P. Luksch (FIZ); K. Rajan, X. Li, A. Rajagopalan (RPI) 


\section{Advanced Measurement Capabilities}

\begin{abstract}
The special role that NIST plays in measurement metrology and standards often results in the development of instruments and facilities, as well as measurement methods, capable of probing material characteristics in ways unavailable elsewhere. These capabilities often provide the context and observations necessary to the development of a fundamental understanding of the mechanisms of material behavior.

The Ceramics Division is privileged to have an excellent staff and world leaders who are responsible for the design and development of many of these unique instruments and facilities.
\end{abstract}

\section{Debra L. Kaiser}

\section{Nanometrology}

W orking with instrument makers, the Nanotribology Group, directed by Dr. Stephen Hsu, has designed and built a remarkable facility featuring a cluster of equipment and instrumentation that establishes a new state of the art for conducting measurements and observations of nanoscale events. Included in this facility are a nanoadhesion apparatus, a dual white-light interferometer microscope, a multiscale friction tester, and a multifunction apparatus combining an ultrahigh vacuum scanning tunneling microscope with an atomic force microscope (UHV STM/AFM). Together, these instruments allow us to image, manipulate, and measure adhesion, friction, and other nanomechanical properties of materials from microscale to nanoscale dimensions.

\section{Nanoadhesion Apparatus}

Adhesion is an important issue in nanotechnology as well as in device manufacturing industries. Conventional adhesion measurement relies on large interfacial areas and the accurate positioning of two surfaces. Force measurements typically are performed by strain gauges and transducers with force sensitivities in the newton and millinewton ranges. These

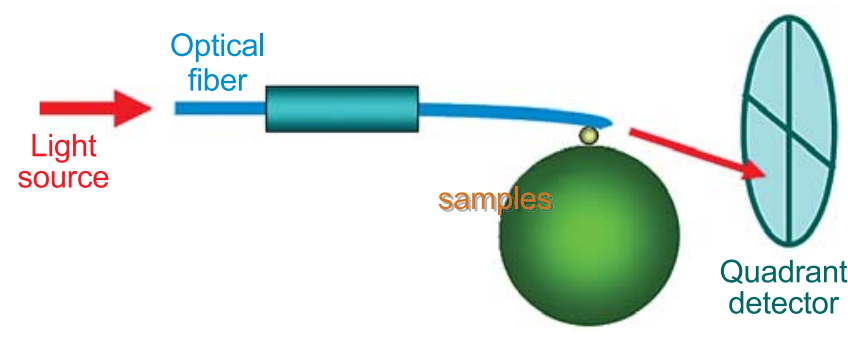

Figure 1: Schematic operation of the NIST nanoadhesion apparatus. conventional instruments are unsuitable for nanoscale contacts. To resolve this issue, the newly designed NIST apparatus, Figure 1, allows adhesion force measurements between two surfaces at the nanoscale level using the AFM force measurement principle. In this device, capable of nanonewton force resolution, a laser light is piped through a fiber-optic cable directed at a quadrant photodetector. Because the length and stiffness of the fiber can be modified easily, a wide range of spring constants can be obtained, and the measurement of adhesion forces can be achieved at multiple force levels. Thus, the NIST apparatus has established an exciting new opportunity to measure the influence of surface forces on adhesion, molecular interactions, and the compliance of surfaces, all critical data to device manufacturing industries.

\section{UHV STM/AFM}

Enhanced by the superior vibration isolation and clean room environment in NIST's new Advanced Measurement Laboratory (AML), our UHV STM/AFM system, Figure 2, provides atomic imaging and force measurement with an unprecedented resolution and accuracy.

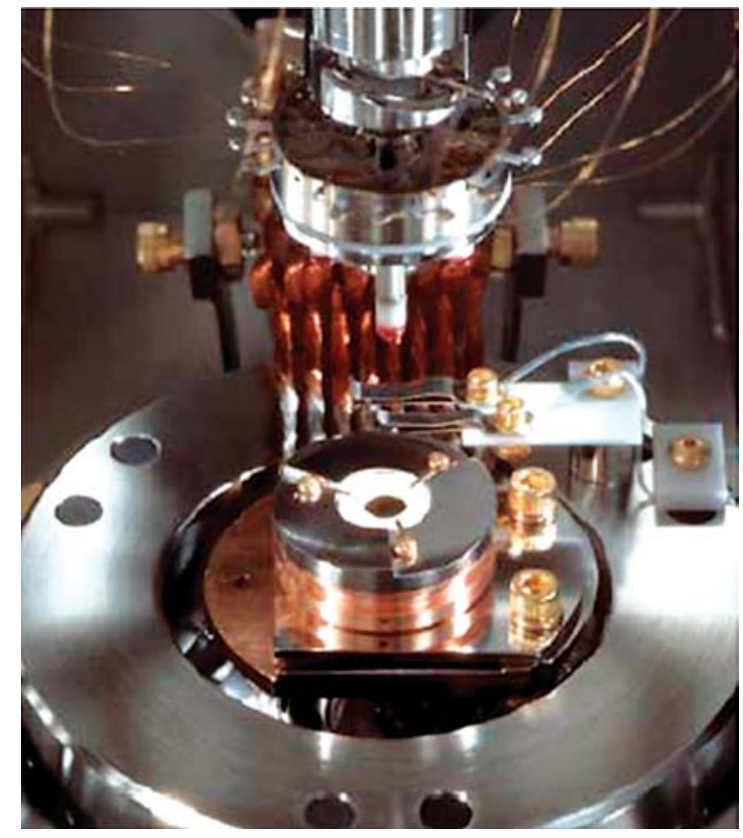

Figure 2: Ultrahigh vacuum STM/AFM apparatus

Combined with our conventional AFM, equipped with a "triboscope" attachment, we are able to image and manipulate surface features and measure a wide range of material characteristics and properties important in nanodevice operation. As a result, we now are leading fundamental metrological efforts, working with device 
and magnetic hard disk industries, as well as academic institutions, to establish reliable measurement methods, calibration artifacts, and infrastructural support for current and future industries.

\section{Multiscale Friction Tester}

At the nanoscale level, friction between movable components is a significant concern. To address this issue, NIST and Hysitron, Inc. have collaborated to develop a three-dimensional, capacitance based, force sensor. The stiffness and position of the probe tips can be tightly controlled to yield accurate information of the contact area. Force measurements can be made in the range from $100 \mathrm{nN}$ to $1 \mathrm{~N}$ in the $\mathrm{z}$-direction, and $500 \mathrm{nN}$ to $0.5 \mathrm{~N}$ in the $\mathrm{x}, \mathrm{y}$-directions. Displacements can be measured from $0.2 \mathrm{~nm}$ to $5 \mu \mathrm{m}$ in the z-direction, and $10 \mathrm{~nm}$ to $15 \mu \mathrm{m}$ in the $\mathrm{x}, \mathrm{y}$-directions. Consequently, accurate friction measurements can be conducted at length scales ranging from the nanometer scale to the micrometer scale. Already, this instrument has enabled us to resolve apparent disparities in nanofriction measurements and to understand the nature of what had been thought to be anomalous friction behavior. (See Friction Scaling in the Technical Highlights section of this report.)

\section{High Resolution X-ray Metrology}

Powder and single crystal x-ray diffraction are widely used in industry, research facilities, and academia as one of the principal means of characterizing materials. Both techniques yield a wealth of information on the crystallographic and microstructural character of the specimen. The powder diffraction method has the virtue that it can probe a continuous sequence of crystallographic reflections with a single scan in angular space, while single crystal methods can be adapted to high resolution analysis of materials such as thin films.

However, results from both powder and highresolution techniques are affected by a complex optical aberration function that is specific to the diffraction optics and goniometer assembly used in the experiment. NIST Standard Reference Materials (SRMs) are the recognized means by which these aberrations may be characterized to achieve improved measurement accuracy. To address these issues, Dr. James Cline of the Data and Standards Technology Group conceived and designed the Ceramics Division Parallel Beam Diffractometer (CDPBD).

The CDPBD, Figure 3, was designed and built specifically to perform traceable measurements on powder and thin film specimens. Installed in the new NIST Advanced Measurement Laboratory, this facility provides the environmental and temperature controls requisite for a new generation of NIST SRMs that will enable unprecedented measurement accuracy in a highly competitive, data conscious, materials research community.

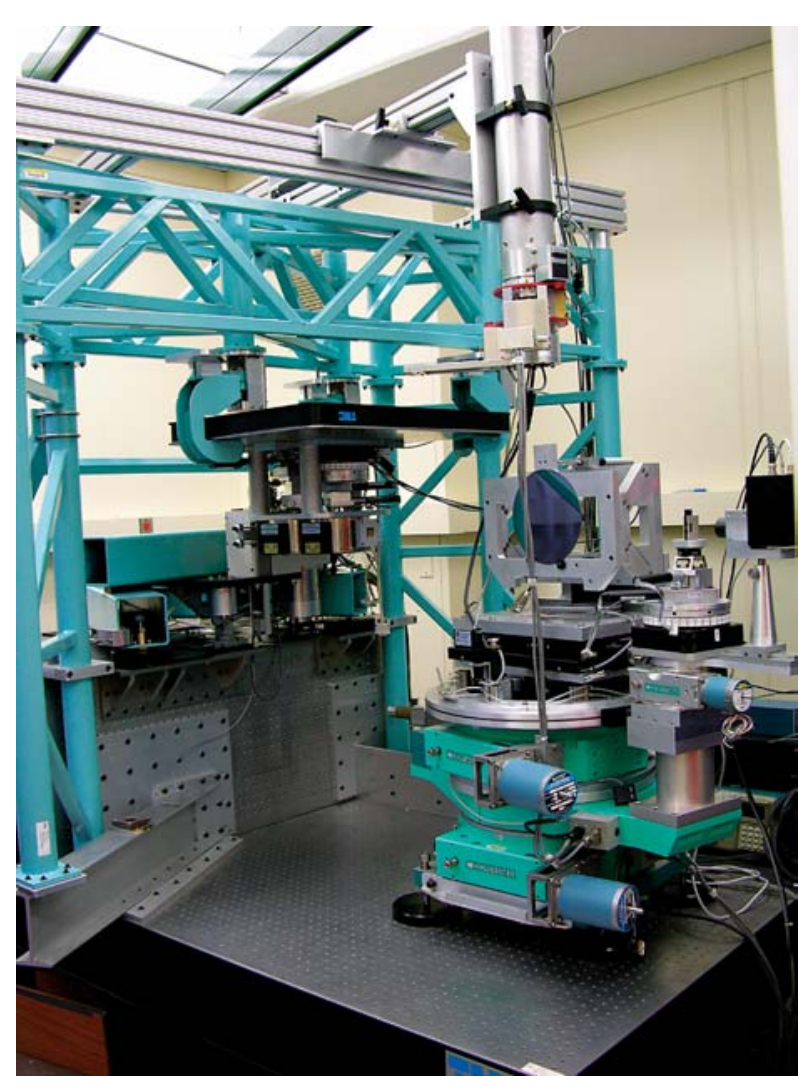

Figure 3: The Ceramics Division Parallel Beam Diffractometer in the $A M L$.

\section{X-ray Absorption Spectroscopy}

Advances in our x-ray absorption spectroscopy facilities have achieved an unrivaled capability enabling our Characterization Methods Group (CMG) to address a remarkably broad range of challenging structure and chemistry issues at the forefront of materials science research today. Through the application of a truly unique combination of beamline facilities, we are able to examine characteristics of surfaces, interfaces, and bulk materials in a manner heretofore inaccessible.

To achieve this capability, CMG, led by Dr. Daniel Fischer, brought together a suite of three unique highthroughput $\mathrm{x}$-ray spectroscopy beamlines (designated U7A, X24A, and X23A2). Housed in the National Synchrotron Light Source located at Brookhaven National Laboratory in New York, these beamlines, taken together, can easily examine nearly all of the naturally occurring elements in the entire periodic table. This year, the capabilities of beamline U7A, used for soft x-ray materials science applications, were significantly enhanced by the addition of a 14 element, state-of-the-art, $\mathrm{Si}(\mathrm{Li})$ fluorescence yield detector, providing best in the world resolution. Further enhancement of U7A, Figure 4, was achieved through the installation of a 6-axis manipulator that enables the exciting prospect of molecular alignment studies. 


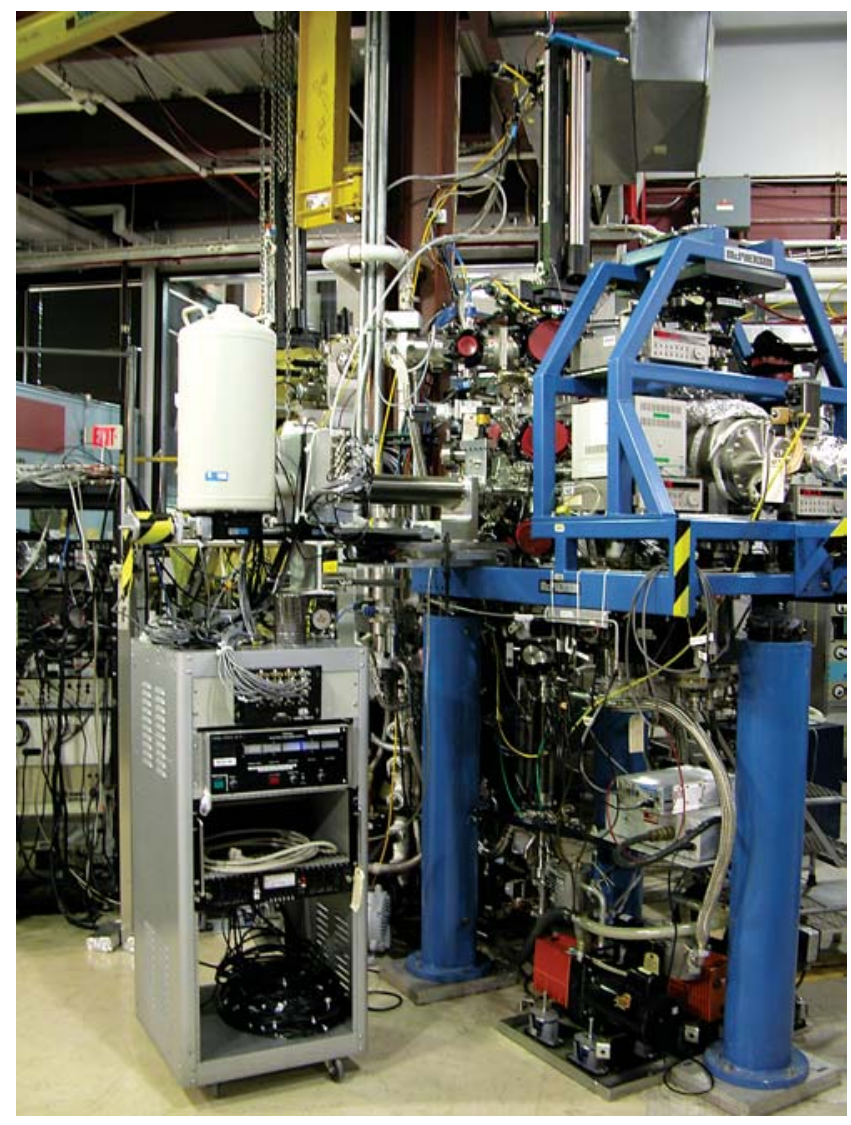

Figure 4: The soft x-ray spectroscopy station on beamline U7A.

Building on these capabilities, CMG has initiated a long-term plan, co-funded with Sandia National Laboratory, for establishing a variable energy XPS (x-ray photoelectron spectroscopy) and NEXAFS/EXAFS (near-edge/extended x-ray absorption fine structure) scientific program utilizing beamline X24A. A new, fully automated materials science end-station is planned, modeled after the very successful high throughput attained on U7A. The emphasis for this work will be on the use of variable energy XPS for chemical depth profiling, sub-surface chemistry, and interface chemistry.

\section{X-ray Topography}

The premier, dedicated, monochromatic topography facility in the U.S. has been developed and implemented at the Advanced Photon Source (APS) at Argonne National Laboratory (ANL), Argonne, Illinois. X-ray topography is used to study the defect microstructure of single-crystal materials by imaging the diffracted intensity from selected lattice planes of the sample. The NIST effort, led by Dr. David Black, takes advantage of the third-generation synchrotron $\mathrm{x}$-ray source to provide a spatially enlarged beam, up to $140 \mathrm{~mm}$ x $50 \mathrm{~mm}$, with large-area real-time imaging detectors to perform static and dynamic experiments with large samples. In addition to topography, radiographic and phase contrast imaging are available. This instrument has recently been used to study subsurface damage in $300 \mathrm{~mm}$ production silicon wafers, defects in thin strained-silicon films, strain around nanoindentations and dendrite growth in $\mathrm{Sn} / \mathrm{Bi}$ alloys. In the case of the strained-silicon films, by using grazing incidence geometry we have been able to directly image defects in a film $\approx 165 \AA$ thick. These defects have been shown to mimic the misfit dislocation structure in the underlying relaxed $\mathrm{Si} / \mathrm{Ge}$ virtual substrate. Understanding how the substrate defects propagate into the strained-silicon film is a critical issue in the development of the next generation of strained silicon electronic devices.

\section{Ultrasmall-Angle X-ray Scattering}

Our ultrasmall-angle x-ray scattering (USAXS) instrument, constructed as part of the UNICAT facility, is housed at the APS at ANL. The NIST effort is led by Dr. Andrew Allen. The facility provides the most versatile SAXS facility for materials science and engineering research in the world. Using a single instrument configuration, the representative microstructures within a wide range of heterogeneous materials of technological importance can be quantified over a scale range extending from nanometers to micrometers. The continuously tunable x-ray energy enables anomalous SAXS studies to be made, allowing different microstructural components within a composite system to be distinguished. Microstructures also can be measured when they possess an arbitrarily high anisotropy, such as those encountered in some coating materials and membranes. Recently, the high APS brilliance has been combined with the instrument's high-precision x-ray crystal optics to provide a spatial resolution on the order of $10 \mu \mathrm{m}$ for resolving the microstructural gradients within solid oxide fuel cell (SOFC) components.

The USAXS instrument serves microstructure metrology needs in a wide class of technological problems encountered in metals and alloys, structural ceramics, thermal barrier coatings, fuel cells, biological scaffolds, flowing nanoparticle suspensions, soot in flames, and polymer gels. Furthermore, the intrinsic absolute intensity calibration provided by this instrument underpins our partnerships with others to develop specialized SAXS instrumentation for interrogating surface structures in electronic gate materials, or to approach sub-micrometer spatial resolution in SOFC research.

\section{For More Information on this Topic}

S. Hsu, J. Cline, D. Fischer, J. Woicik, D. Black, A. Allen (Ceramics Division, NIST) 


\section{Outreach for Advanced Technology Dissemination}

The core mission of NIST is focused on the needs of U.S. industry and commerce. That focus leads naturally to metrological capabilities having substantial application and benefit not only to industry, but also to every federal agency utilizing advanced technology in its operations. Thus, NIST has accrued a long history of providing significant scientific and technical support to the missions of federal and private sector jurisdictions beyond the Department of Commerce.

\section{Debra L. Kaiser}

$\mathrm{T}$ he Ceramics Division is a strong participant in mission support activities. Current research projects within our Division address issues of importance to numerous agencies, including the Departments of Defense, Energy, and Homeland Security, the U.S. Mint, the National Science Foundation, and the International Energy Association, as well as consortia involving industry and academia.

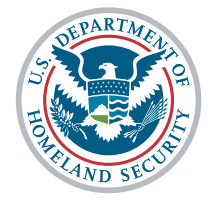

\section{Customs and Border Protection (DHS)}

The U.S. Customs and Border Protection (CBP) is tasked with the responsibility of classifying ceramic imports such as ceramic wares for laboratory, chemical or other technical uses. This category encompasses most technical, high-performance, or advanced ceramic products including engine components, bearings, and machine tools. The classification of these articles is important because the classification determines the import duty rates. Consequently, CBP needs reliable assessment tools capable of accurately determining the classification of the incoming ceramic parts. Chapter 69 of the Harmonized Tariff Schedule of the United States, subheading 6909.12, describes these articles as having a hardness equivalent to 9 or more on the Mohs scale. However, the Mohs hardness test is a classical mineralogical scratch test that is not sufficiently accurate for determining the hardness of advanced ceramics. To resolve this issue, the U.S. Customs Laboratory in San Francisco worked with Division staff to identify a more appropriate test. Building on the Division's expertise in standards organizations and independent studies, the NIST-Customs team determined that a $15 \mathrm{GPa}$ Knoop hardness test with an indentation load of $9.8 \mathrm{~N}$ would provide the advanced ceramic equivalent to the Mohs hardness specification in the Tariff Schedule. The necessary reliability of the test was assured by the availability of NIST Standard Reference Material, SRM 2830, Knoop Hardness of Ceramics. This SRM has a certified hardness of $14 \mathrm{GPa}$ and is ideal for the verification of proper machine and operator application of the test. As a result of this collaboration, DHS prepared an important new standard for its customs operations, "U.S. Customs Laboratory Method 69-nn, Procedure for Measuring the Hardness of Technical Ceramic Products."

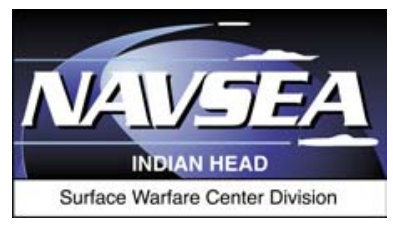

\section{U.S. Navy (DOD)}

In work funded by the Indian Head Division of the Naval Surface Warfare Center, Dr. G. Piermarini (Guest Scientist, NSWC/IHDIV, Research and Technology Department, Indian Head, Maryland), is studying nano-structured reactive materials using a high-temperature, high-pressure diamond anvil cell developed at NIST. By sintering oxygen-passivated nano-metals mixed with oxide mixtures, under suitable conditions of pressure and temperature, it may be possible to prepare a new class of composite materials that have steel-like or ceramic-like properties, but having the energetic reactivity of thermites. If successful, this technology transfer will enable the development of new materials tailored to naval and other defense operations needs.

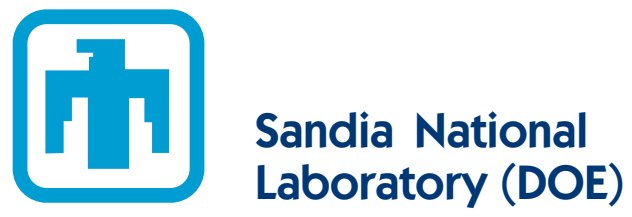

The Characterization Methods Group has initiated a long-term plan co-funded with Sandia National Laboratory for establishing variable kinetic energy XPS for chemical depth profiling, and sub-surface and interface chemistry for diverse materials science problems. This new DOE partnership compliments our existing, very successful collaboration with the Brookhaven National Laboratory's Materials Science Department focused on the electronic structure of high-temperature superconductors and lithium battery oxides. 


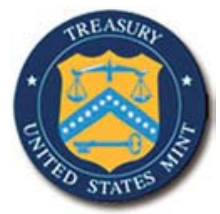

\section{U.S. Mint}

The Nanotribology Group is assisting the U.S. Mint to enhance their control of coin fabrication processes and surface finishes. With millions of coins in circulation and millions of coin-operated devices, it is essential to the mission of the U.S. Mint that the coins be both durable and consistent in their fabrication. Our specialized instrumentation enables a guest scientist from the U.S. Mint to assess the surface chemistry and surface properties of coins produced by various fabrication processes.

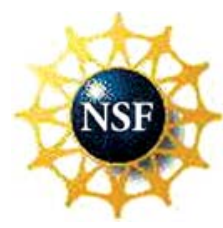

\section{National Science Foundation (NSF)}

The MSEL Summer Undergraduate Research Fellowships (SURF) program provides a joint opportunity for NIST and NSF to provide undergraduates with hands-on, rigorous research experiences in diverse areas of materials research encompassing ceramics, metallurgy, polymers, and measurement science. A core goal is to open these opportunities to women, under-represented minorities, and Americans with disabilities. The program's objective is to reach out at a critical point in the careers of these under-represented students, who have often been discouraged from careers in science, math, and engineering. SURFing the Materials Science and Engineering Lab has made progress toward these goals by forging partnerships with colleges and universities that demonstrate strong hands-on undergraduate science curricula and a commitment to the education of women, minorities and students with disabilities. The MSEL SURF program was initiated in 1998 and is one of seven spokes of the now site-wide activity, SURFing the NIST Laboratories. The program has grown to include approximately one hundred students each summer. The overall demographic trends for the program are: $48 \%$ female, $52 \%$ male; $13 \%$ African-American, $12 \%$ Hispanic, and $14 \%$ Asian.

\section{CRADA}

This year, the Characterization Methods Group and the Dow Chemical Company celebrate the tenth anniversary of a CRADA to establish and foster a soft x-ray synchrotron radiation materials science characterization facility (beamline) at the National Synchrotron Light Source. Strategic research in catalysis, polymers and polymer surfaces has been enabled by the joint long-term commitment to new detector development and high-throughput capabilities.

\section{Consortium}

As part of the Information Storage Industry Consortium (INSIC), the Nanotribology Group collaborates with 28 companies and 41 universities, research institutes, and government laboratories to provide nanotribology test methods that will enable the development of ultra-high density storage technology. We have participated in the Tribology Working Group and have contributed to the development of technology roadmaps to guide the development of next-generation storage technology.

\section{Versailles Project on Advanced Materials and Standards (VAMAS)}

The Nanotribology Group has taken the lead in organizing the first international round-robin study on how to calibrate cantilever spring constants in atomic force microscopes (AFMs). In collaboration with research laboratories in the UK, Germany, and Japan, this working group on nanomaterials will determine an initial calibration methodology and its associated statistics. Then the effort will expand to other countries and participants around the world leading to the development of consensus standards and calibration artifacts.

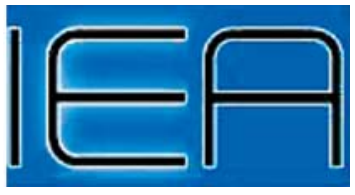

\section{International Energy Agency (IEA)}

DOE and NIST have signed an interagency agreement to cooperate on pre-standardization research focused on critical issues in the metrology of surfaces. As part of that effort, the Nanotribology Group is organizing an international round robin focused on information exchange and cooperative research regarding surface texturing, thin film coatings, and appropriate surface chemistry. The work aims to provide the basis for international standards that will contribute to the design of engine components with reduced friction and increased durability and, hence, will promote the conservation of energy. This project is sponsored by the U.S. Department of Energy, Office of Transportation Technologies.

\section{For More Information on this Topic}

G. Quinn, D. Fischer, S. Hsu, T. Vanderah (Ceramics Division, NIST) 


\section{Friction Scaling Artifact}

Nanofrictional forces are an important factor in the design of nanodevices and assemblies of nanoparticles. Literature data on nanofriction, however, exhibit many discrepancies and have a wide range of scatter. In a series of well-controlled experiments, we have discovered that a significant portion of this discrepancy may actually be attributed to an unintended scratching of the surface by the sharp tips used in making the measurements themselves. Deviation of tip shape from the "ideal" spherical shape is another contributor to the disagreements found among the literature data. This work sheds important insight into the nature of nanoscale friction measurement.

\section{Z. Charles Ying and Stephen M. Hsu}

\begin{abstract}
$\mathrm{A}^{\mathrm{c}}$ ccurate measurement of nanofriction is emerging as one of the critical issues in nanotechnology. Manipulation of molecules, clusters, and nanoparticles across surfaces to form a functional entity relies on the ability to overcome the resistance of the particles to motion across the surface. Further, reliable operation of micro- and nano-devices often depends on an accurate estimate of lateral loading. Literature data on nanofriction, however, have shown wide ranges of values depending on the length scale of the device. Silicon friction, e.g., shows an order of magnitude variation from 0.03 to 0.25 . Such results have led to speculations that there might be an intrinsic scaling effect such that different friction levels would occur across the nano-, micro-, and macro-scales. If proven true, well established friction laws would be invalidated for nanomaterials.
\end{abstract}

Given the broad technological significance of this issue, we have undertaken a detailed investigation of the nature of this discrepancy. Friction measurements between non-adhering surfaces were carried out in both elastic and plastic deformation regions. Diamond tips of various sizes were used to measure nanofriction forces on well-characterized substrates such as single crystal silicon, silicon dioxide, and calcium fluoride. Experiments were performed using a specially designed multiscale friction tester developed jointly by NIST and Hysitron Incorporated based on a capacitance probe force transducer. In each experiment, the environment was carefully controlled, and the tip penetration into the substrate during sliding was continuously measured.

The size and shape of each tip were characterized using a newly developed "replica technique." Tips were imprinted into a soft material, and the imprints were scanned by AFM and digitally inverted to
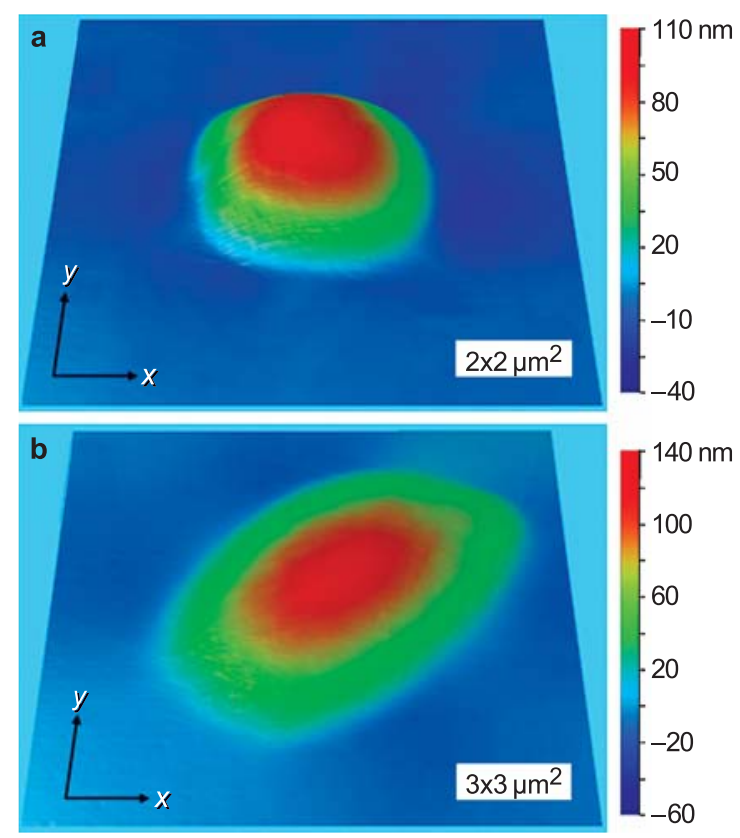

Figure 1: 3D profiles, obtained using the replica technique, of the tips used in the friction measurements.

"reconstruct" the tip in three-dimensional details. Figure 1 shows images of two of the diamond tips used in the experiments. The tip shown in Figure 1(a) is spherical in shape with a $1.2 \mu \mathrm{m}$ tip radius while Figure 1(b) shows an ellipsoidal shape with nominal radius of $4 \mu \mathrm{m}$. The detailed dimensional data are crucial in determining the apparent contact areas and isolating the influence of surface forces.

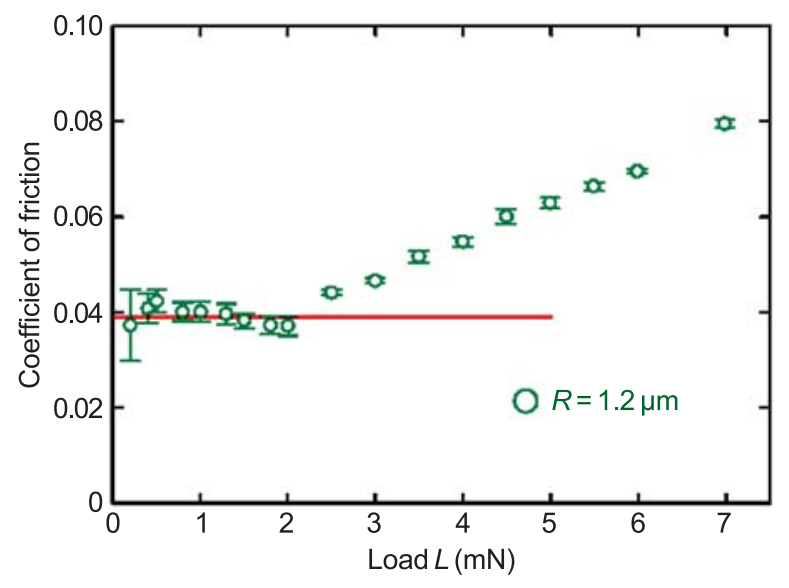

Figure 2: Coefficient of friction between Si (100) surface and a diamond tip with radius $\mathrm{R}=1.2 \mu \mathrm{m}$, as a function of mechanical loading force.

Figure 2 shows the coefficient of friction (COF) between a Si (100) surface and a diamond tip with tip radius $R=1.2 \mu \mathrm{m}$. 


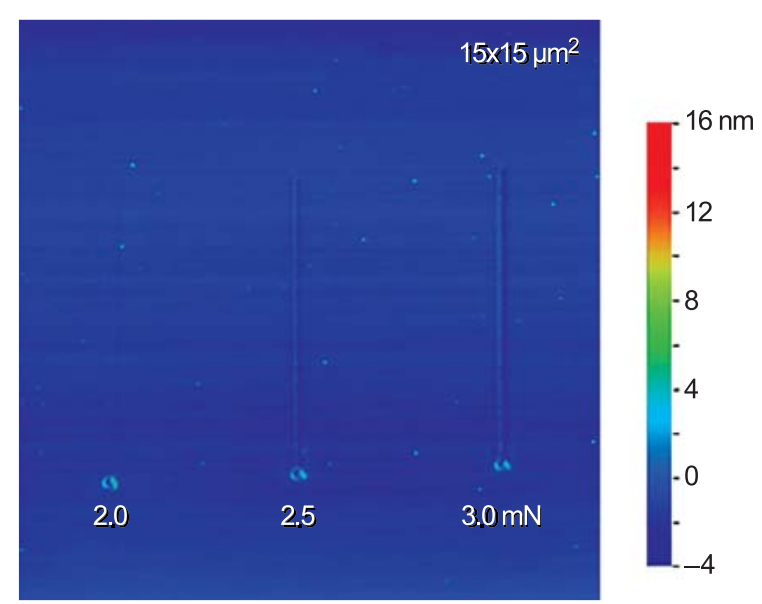

Figure 3: Image of the silicon surface after friction measurements, showing scratches at $2.5 \mathrm{mN}$ and higher load.

Two distinct regions can be seen. Initially, the COF is constant up to a load of $2.0 \mathrm{mN}$. For loads above $2.5 \mathrm{mN}$, the COF increases with load. This friction transition is contrary to Amoton's law. Subsequent investigation revealed that this change in COF is caused by unintended plowing of the tip into the substrate. Atomic force microscopy, using a sharp tip on the surface, suggests that the COF increase coincides with the first appearance of plastic grooving. In the case of silicon, a groove $5 \AA$ deep and $2000 \AA$ wide was detected on the surface at a load of $2.5 \mathrm{mN}$ (Figure 3).

This observation was repeated for different tips and different substrates. At higher loads, deeper and wider grooves were observed. These results suggested that unintended scratching of the surfaces by the sharp tips used in the friction measurements contributed to the discrepancies among friction data in the literature.

The initiation of plastic grooving can be quantified in terms of contact pressure. By determining the apparent contact area using the digitally inverted tip method, the transition contact pressures could be calculated for all of the observed cases. All the results corresponded to $12 \mathrm{GPa}$ which is approximately the hardness of silicon.

\section{Elastic Plowing}

Figure 4 shows the friction data for three tips in the elastic region (no plastic grooving). The data for the two spherical tips with radii $R=0.5 \mu \mathrm{m}$ and $1.2 \mu \mathrm{m}$ exhibit a common value for the COF. However, a higher friction value was observed for the $4 \mu \mathrm{m}$ radius tip.

To explain this discrepancy, we tested the various contact mechanics models which require the accurate determination of the real contact areas. Considering a tip plowing through an elastic substrate, the real area of contact is the projected cross-section area of the tip in the direction of sliding. This can be determined from the tip geometry and "penetration depths" across the sliding path. Numerical integration of actual tip size and shape over the sliding path is required. For the hypothesis of "elastic plowing," the calculation shows that the friction force in this regime should be proportional to the projected cross-sectional area in the direction of sliding. Figure 5 confirms this hypothesis for all three tips.

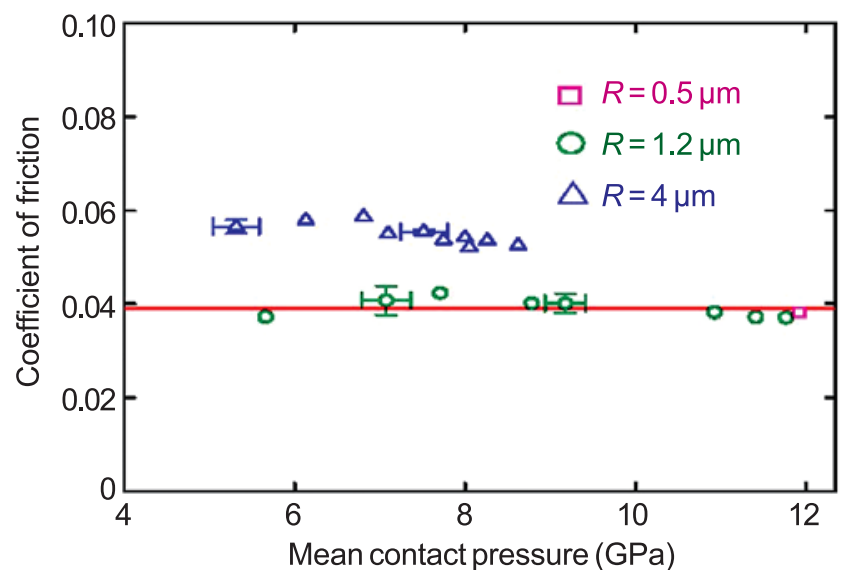

Figure 4: Coefficient of friction between Si (100) surface and three diamond tips used in the experiments, as a function of mean contact pressure.

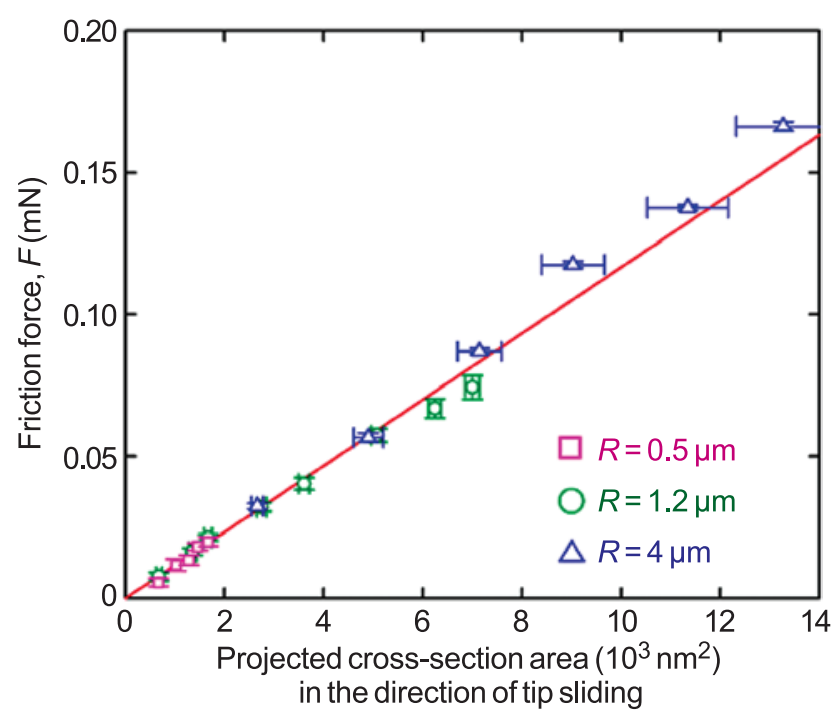

Figure 5: Friction force as a function of projected cross-section area in the direction of tip sliding.

This work shows clearly that nanoscale friction, in practice, is a complex result of both elastic and inelastic strains and deformations, and accurate nanofriction measurement will require careful accounting of all the forces contributing to the phenomenon. The methodology established in this work provides a substantial step towards the data and understanding needed by industry.

\section{For More Information on this Topic}

\section{Z.C. Ying, S.M. Hsu (Ceramics Division, NIST)}




\section{First-Principles Studies of Technologically Important Dielectric Materials}

\begin{abstract}
Dielectric materials are needed for diverse applications ranging from gate dielectrics to microwave resonator materials to transducer and actuator materials. The specific materials requirements depend on the application. First-principles theoretical studies on dielectric materials are revealing the roles of chemical order and internal electric fields in controlling the properties of these materials.
\end{abstract}

\section{Eric Cockayne and Benjamin P. Burton}

Current semiconductor technology uses amorphous $-\mathrm{SiO}_{2}$ as a gate dielectric material. As size has decreased, however, the leakage current of $\mathrm{SiO}_{2}$ has become a limiting factor. Finding an alternative gate dielectric material is a "grand challenge" in the industry. Alternative materials must have higher dielectric constants than $\mathrm{SiO}_{2}$ to yield equivalent performance with reduced leakage current. Hf-containing oxides are a promising set of materials.

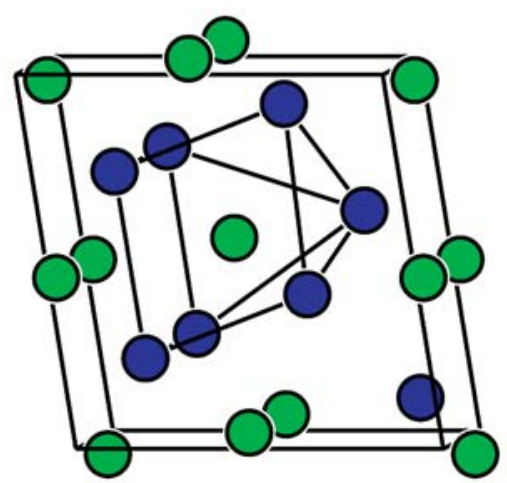

Figure 1: $\mathrm{HfO}_{2}$ structure (Hf green, $\mathrm{O}$ blue).

Dielectric constants are related to the effective charges of ions and to phonon-frequencies. First-principles (FP) calculations show that the effective charge of $\mathrm{Hf}$ in $\mathrm{HfO}_{2}$ (Figure 1) is higher than the effective charge of $\mathrm{Si}$ in $\mathrm{SiO}_{2}$, providing an explanation why Hf-containing oxides should have, in general, higher dielectric constants than silicates. By comparing the effective charge of $\mathrm{Hf}$ in the experimental structure with that in hypothetical structures, it was found that the effective charge of $\mathrm{Hf}$ depends on its environment. Because amorphous gate dielectrics are desirable, knowledge of how crystal environments dictate effective ion-charges provides a basis for designing gate dielectric materials.

Microwave resonator and filter materials typically require a moderate dielectric constant, low dielectric loss,

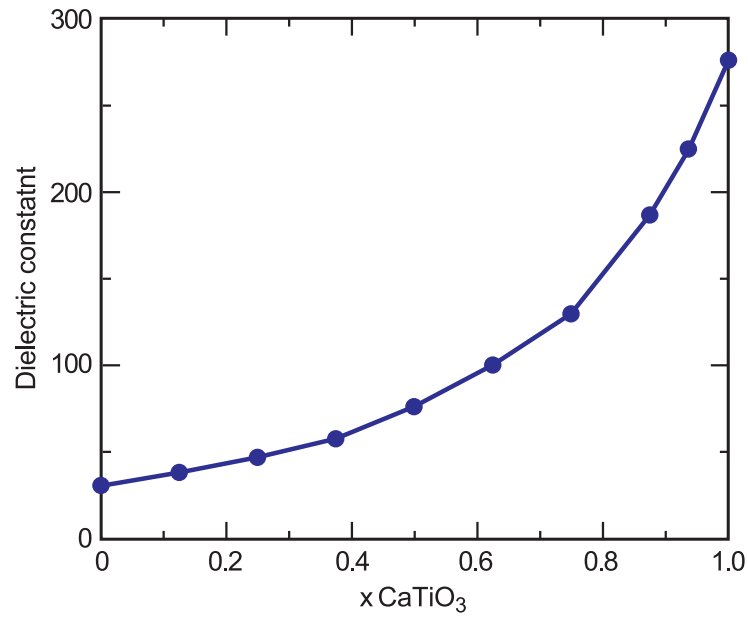

Figure 2: First-principles-derived model for the dielectric constant of $\left(\mathrm{CaAl}_{1 / 2} \mathrm{Nb}_{1 / 2} \mathrm{O}_{3}\right)_{1-x}-\left(\mathrm{CaTiO}_{3}\right)_{x}$ shows nonlinear composition dependence, in agreement with experiment.

and temperature stability. In general, temperature stability is achieved by forming solid solutions between end member compounds with opposite signs of temperature coefficient, for example, between $\mathrm{CaAl}_{1 / 2} \mathrm{Nb}_{1 / 2} \mathrm{O}_{3}(\mathrm{CAN})$ and $\mathrm{CaTiO}_{3}(\mathrm{CT})$. We have used the FP-based model to calculate the dielectric constant of arbitrary solid solutions of $-\mathrm{CaAl}_{1 / 2} \mathrm{Nb}_{1 / 2} \mathrm{O}_{3}-\mathrm{CaTiO}_{3}$. The model is based on cluster expansions of the interatomic forces and ionic charges, which control the dielectric properties.

The model reproduces the experimentally measured composition dependence of the dielectric constant of CT-CAN very well (Figure 2).

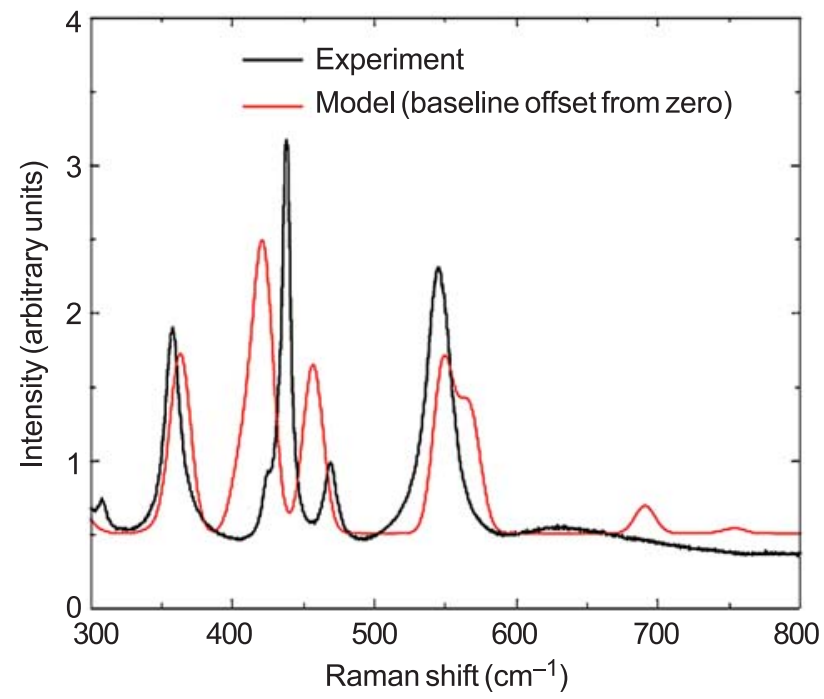

Figure 3: Combined first-principles/empirical model for the Raman spectrum of $\mathrm{CaZrO}_{3}$ shows semiquantitative agreement with experiment. 
Dielectric properties, and, therefore, the Raman spectra, of solid solutions depend on chemical ordering of ions as well as composition. To understand the connection between local structure, composition, dielectric properties, and spectroscopy, we are working on computing Raman spectra directly from FP. The idea is to create a spectroscopic/computational tool for measuring short- and long-range chemical order via Raman spectroscopy. Experiments have shown that Raman spectra are sensitive to short- and long-range order, but little is known quantitatively about the relationship between Raman spectra and chemical order. We have created a model, based on FP results, for the Raman spectra of solids, and are applying it to study the CAN-CT and $\mathrm{CaZrO}_{3}-\mathrm{CaTiO}_{3}$ (CZ-CT) solid solution systems. Figure 3 shows that the Raman spectrum of $\mathrm{CZ}$ is reproduced reasonably well with only a few parameters. Both long-range electrostatics and short-range covalent bonding contribute to the Raman spectra, but not equally to all peaks. This provides a natural explanation for the experimental observation that different Raman peaks can have different sensitivities to the amount of long-range order. Evidence is also found that small $\mathrm{Ti}$ or $\mathrm{Zr}$ concentrations create local vibrations that produce significant, but broad, peaks in the Raman spectrum.

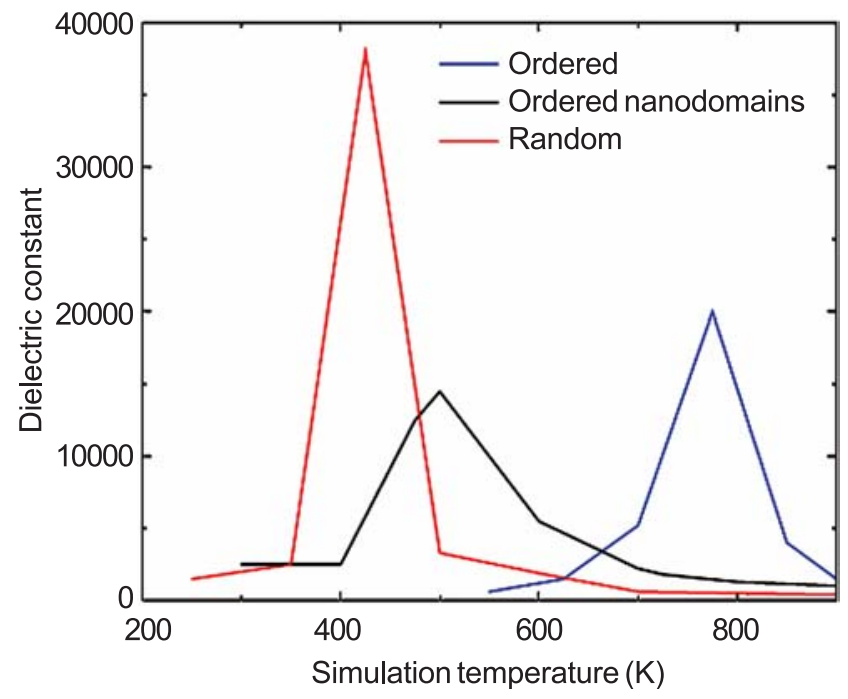

Figure 4: Simulated dielectric constant of $\mathrm{PbSc}_{1 / 2} \mathrm{Nb}_{1 / 2} \mathrm{O}_{3}$ as a function of temperature and type of $\mathrm{Sc}-\mathrm{Nb}$ ordering. The broadening of the dielectric peak that occurs in the case of ordered nanodomains is one characteristic of relaxor behavior.

Transducers used for sonar and medical imaging applications typically require extremely high dielectric and piezoelectric coefficients. Very large piezoelectric coefficients are observed in $\mathrm{Pb}[\mathrm{Mg} ; \mathrm{Zn}]_{1 / 3} \mathrm{Nb}_{2 / 3} \mathrm{O}_{3}-$ $\mathrm{PbTiO}_{3}$ solid solutions, based on the relaxor ferroelectrics $\mathrm{PbMg}_{1 / 3} \mathrm{Nb}_{2 / 3} \mathrm{O}_{3}$ and $\mathrm{PbZn}_{1 / 3} \mathrm{Nb}_{2 / 3} \mathrm{O}_{3}$.
Relaxor behavior is desirable, because it implies high-dielectric constants and piezoelectric coefficients over a broad temperature range, but it poses a theoretical challenge because these properties are sensitive to structural/chemical heterogeneities at many length-scales. For example, $\mathrm{PbSc}_{1 / 2} \mathrm{Nb}_{1 / 2} \mathrm{O}_{3}$ (PSN) exhibits normal ferroelectric behavior as a function of temperature (e.g., a sharp dielectric peak) when $\mathrm{Sc}^{3+}$ and $\mathrm{Nb}^{5+}$ are ordered. However, when there is only short-range order, such as nanoscale chemically ordered domains in a disordered matrix, PSN exhibits relaxor ferroelectric behavior (e.g., a broadened, frequency-dependent dielectric peak). Similar changes in dielectric properties are caused by the introduction of $\mathrm{Pb}$ - and $\mathrm{O}$-vacancies. We have constructed a computational model for PSN, which allows these structure-property issues to be explored in detail. FP calculations show that $\mathrm{Pb}$ atoms move off their ideal crystallographic positions, and that the dielectric and piezoelectric response in PSN is dominated by the dynamics of $\mathrm{Pb}$-displacements. At low temperature, $\mathrm{Pb}-\mathrm{Pb}$ interactions favor coherent alignments of $\mathrm{Pb}$-displacements, but local electric fields, caused by the charge difference between $\mathrm{Sc}^{3+}$ and $\mathrm{Nb}^{5+}$, randomly bias individual $\mathrm{Pb}$-displacements. Electric fields generated by $\mathrm{Pb}$ - and $\mathrm{O}$-vacancies are also included in this model. Molecular dynamics simulations on a 320,000 atom supercell yield a sharp dielectric peak for ordered PSN, and successive broadening as chemically ordered nanodomains and/or $\mathrm{Pb}$ vacancies are added, in agreement with experiment.

The wide band-gap semiconductor system AlN-GaN is used to make light emitting diodes, and the wavelength of emitted light is adjusted by alloying. First-principles phase diagram calculations for this system predict the expected miscibility gap when the excess vibrational entropy is excluded; but when it is included, an ordered phase based on $\mathrm{AlGaN}_{2}$ stoichiometry is predicted. To our knowledge, this is the first prediction of vibrational-entropy induced atomic ordering, and it suggests a novel way to affect band gaps: via ordering and alloying rather than alloying alone.

\section{For More Information on this Topic}

E. Cockayne, B.P. Burton (Ceramics Division, NIST)

E. Cockayne, "First-principles Calculations of the Dielectric Properties of Perovskite-Type Materials," J. Eur. Ceram. Soc. 23, 2375-2379 (2003).

U.V. Waghmare, E. Cockayne, and B.P. Burton, "Ferroelectric Phase Transitions in Nano-scale Chemically Ordered $\mathrm{PbSc}_{0.5} \mathrm{Nb}_{0.5} \mathrm{O}_{3}$ using a First-principles Model Hamiltonian," Ferroelectrics 291, 187-196 (2003). 


\section{Anomalous Elastic Behavior for $\mathrm{SrTiO}_{3}$ Thin Films Grown on Si(001) Observed by X-ray Diffraction}

A major driving force of the semiconductor industry is the integration of transition-metal oxides with Si transistor technology. Much of this effort has focused on the development of $\mathrm{SrTiO}_{3}$ thin-film growth on Si(001). These films have served successfully as the gate-oxide layer for metal-oxide field-effect transistors and as the buffer layer for III-V on Si semiconductor technology. In addition, ferroelectric thin films show promise for quantum computing applications. However, these perovskite films exhibit unusual features, and it is imperative for future advances that the fundamental aspects of the thin-film epitaxy be understood from both an experimental and a theoretical view. To address this issue, we are studying $\mathrm{SrTiO}_{3}$ as a model material representative of the larger class of candidate oxides with the perovskite structure.

\section{Joseph C. Woicik}

$\mathrm{W}$ e have performed high resolution x-ray diffraction measurements at the UNICAT beamline facility at the Advanced Photon Source on a series of $\mathrm{SrTiO}_{3}$ thin films grown on $\mathrm{Si}(001)$ by Motorola. Figure 1

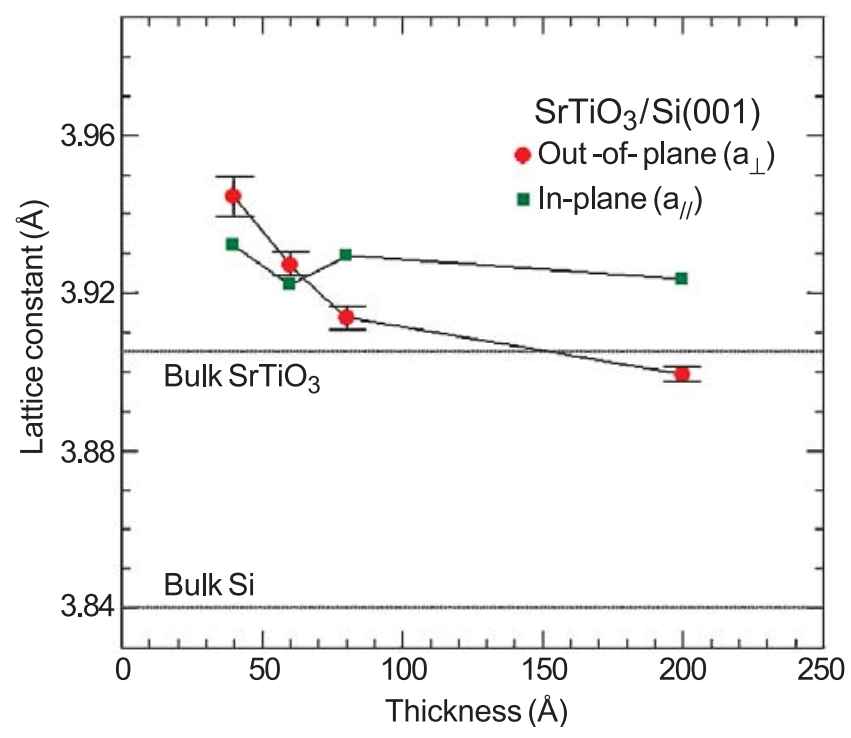

Figure 1: In-plane and out-of-plane $\mathrm{SrTiO}_{3}$ lattice constants for $40 \AA$, $60 \AA, 80 \AA$, and $200 \AA \mathrm{SrTiO}_{3}$ thin films grown on $\mathrm{Si}(001)$. The horizontal lines show the bulk cubic lattice constants of $\mathrm{SrTiO}_{3}$ and bulk $\mathrm{Si}$. shows the results of our glancing-incidence x-ray diffraction experiment performed around the $\mathrm{SrTiO}_{3}(220)$ diffraction for the in-plane and the $\mathrm{SrTiO}_{3}(002)$ diffraction for the out-of-plane $\mathrm{SrTiO}_{3}$ lattice parameters.

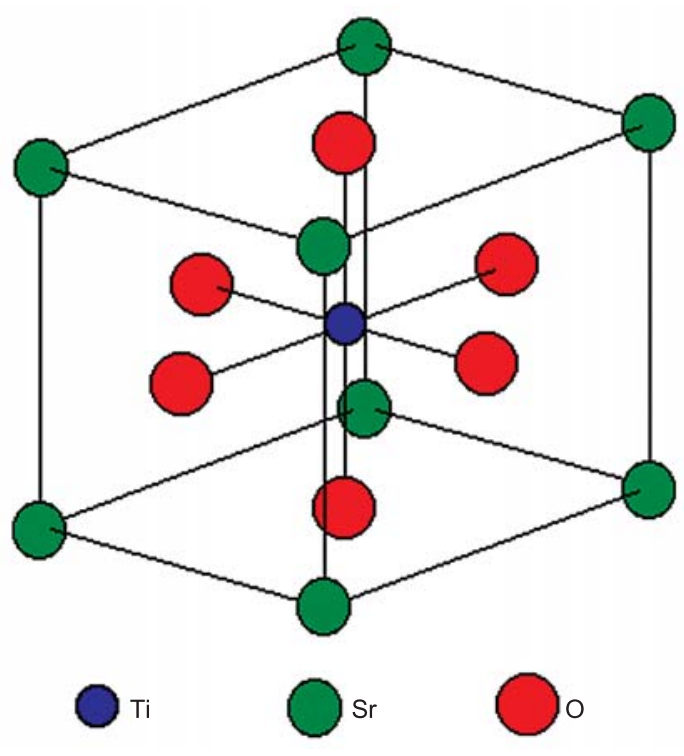

Figure 2: Crystal structure of $\mathrm{SrTiO}_{3}$.

Because the crystal structure of $\mathrm{SrTiO}_{3}$ is simple cubic (Figure 2), whereas the crystal structure of $\mathrm{Si}$ is face-centered cubic, $\mathrm{SrTiO}_{3}$ films grow on the $\operatorname{Si}(001)$ surface with their unit cell rotated $45^{\circ}$ around the $\mathrm{Si}(001)$ surface-normal direction. Consequently, this choice of $\mathrm{SrTiO}_{3}$ reflections determines accurately the $\mathrm{SrTiO}_{3}$ unit cell dimensions relative to the underlying Si lattice and hence the thin film epitaxy.

Our diffraction measurements find that all of the thin films studied are under in-plane tensile strain. This finding is counter to what is expected from the bulk lattice constants of $\mathrm{SrTiO}_{3}$ and $\mathrm{Si}$ that predict in-plane compressive strain for coherent $\mathrm{SrTiO}_{3}$ thin-film growth on $\mathrm{Si}(001)$. The observed expansion of the in-plane $\mathrm{SrTiO}_{3}$ lattice constant must therefore result from the much larger thermal expansion coefficient of $\mathrm{SrTiO}_{3}$, compared to $\mathrm{Si}$, coupled with incoherent growth at the elevated growth temperature. 
Even more surprising is the result that the $\mathrm{SrTiO}_{3}$ out-of-plane lattice constant increases with decreasing film thickness, indicating a large negative Poisson's ratio for the thinnest films; i.e., the out-of-plane lattice constant of the $\mathrm{SrTiO}_{3}$ is expanded more than its in-plane lattice constant.

To understand both the nature and driving force of this anomaly, we performed polarization dependent $\mathrm{x}$-ray absorption fine-structure (XAFS) measurements on the same samples. Together, $\mathrm{XAFS}$ and $\mathrm{x}$-ray diffraction determine uniquely the degree of epitaxy and the local atomic distortions that result.

Our XAFS measurements find significant $\mathrm{p}-\mathrm{d}$ hybridization for the thinner films. This hybridization is allowed only for a non-centrosymmetric distortion of the $\mathrm{SrTiO}_{3}$ unit cell. Polarization-dependent measurements of the Ti-O bond lengths find one Ti-O distance parallel to the $\mathrm{SrTiO}_{3}$ interface and two Ti-O distances perpendicular to it. The Ti-O radial splitting is $\approx 0.22 \AA$. The elastic anomaly observed by diffraction is, therefore, likely driven by the interfacial polarization of the $\mathrm{SrTiO}_{3}$ layers.

To test this hypothesis, first-principles density-functional calculations were performed on periodic $\mathrm{SrTiO}_{3}$ supercells containing $\sqrt{2} \times \sqrt{2} \times \mathrm{N}$ unit cells. The calculations were performed at the Naval Research Laboratory Center for Computational Materials Science. Figure 3 shows the results of the calculations as a function of $\mathrm{N}$, the number of $\mathrm{SrTiO}_{3}$ layers. The in-plane lattice constant of the supercells was constrained to equal the average in-plane lattice constant of the $\mathrm{SrTiO}_{3}$ films as determined by x-ray diffraction. To mimic the polarization of the interface as determined by XAFS, a single Ti-O layer was polarized in the normal direction by constraining its z-coordinates to lie $0.22 \AA$ apart. All other coordinates were fully relaxed.

Remarkably, the out-of-plane lattice constant of the supercells is found to expand in agreement with

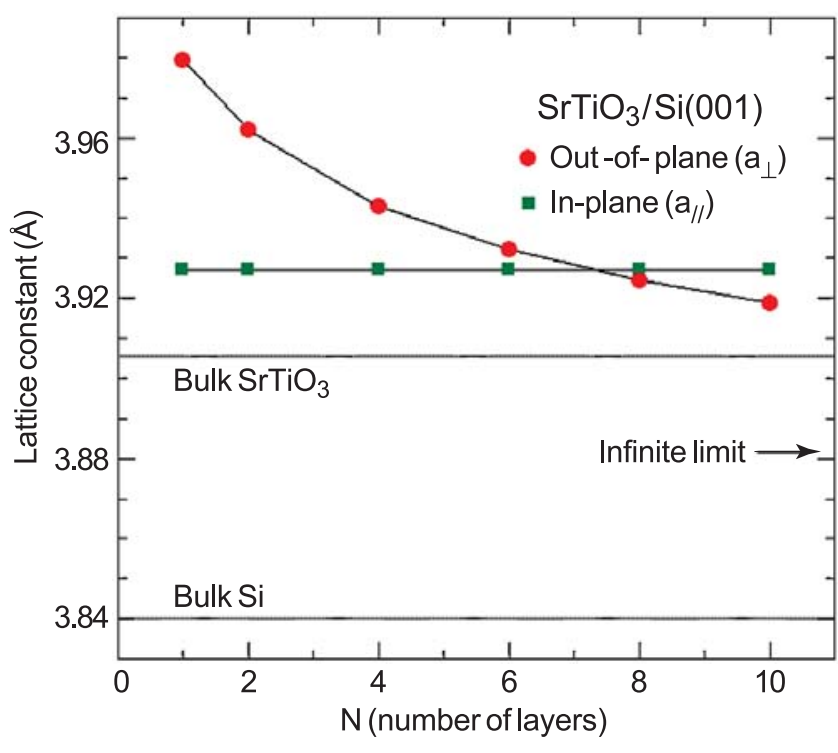

Figure 3: Calculated out-of-plane $\mathrm{SrTiO}_{3}$ lattice constant as a function of the number of $\mathrm{SrTiO}_{3}$ layers.

the measurement of the out-of-plane lattice constant of the films. These results suggest that this behavior may be common for hetero-epitaxial growth of materials that possess an ionic polarizability; it arises simply from the inequivalent perpendicular heights of the anions and cations at the film/substrate interface which drives the polarization of the film.

Additional calculations are being performed to understand the role of $\mathrm{Si}$ diffusion and $\mathrm{O}$ vacancies, and experiments are planned to study both the epitaxy and interfacial structure of films grown under different growth conditions.

\section{For More Information on this Topic}

F.S. Aguirre-Tostado, A. Herrera-Gomez (CINVESTAV); R. Droopad, Z. Yu (Motorola); D.G. Schlom (Pennsylvania State University); P. Zschack, E. Karapetrova (UNICAT); C.S. Hellberg (NRL) 


\section{Mapping Surface Chemistry and Molecular Orientation with Combinatorial Near-Edge X-ray Absorption Fine Structure}

The demands of preparing, processing and characterizing novel materials and structures and the need for full exploration of the typically broad parameter space has led to the development of combinatorial near-edge X-ray absorption fine structure (NEXAFS). This is a new nondestructive synchrotron-based analytical tool for mapping the bond chemistry and molecular orientation of self-assembled monolayer gradients and reaction intermediates in catalyst arrays. Spatially resolved NEXAFS maps reveal bond concentration, rehybridization and orientation of the surfacebound molecules with sub-millimeter planar spatial resolution and sub-monolayer molecular sensitivity.

\section{Daniel A. Fischer and Jan Genzer (NCSU)}

$\mathrm{M}$ ost experimental combinatorial methods concentrate on probing only a single material property, such as chemical information, topography, etc. For some applications, it is desirable that multiple properties be probed simultaneously in a single experiment. Particularly, information on the chemistry and molecular organization of chemical groups on surfaces is needed to shed light on the behavior of amphiphilic surface modifiers or to characterize new classes of catalysts. The chemistry (including bond concentration and rehybridization) and molecular orientation on chemically heterogeneous surfaces and catalyst arrays can be obtained by simultaneously utilizing synchrotron based near-edge X-ray absorption fine structure (NEXAFS) spectroscopy and rastering the soft X-ray beam on the probed specimen (Figure 1). This rastering produces serial NEXAFS images in space and X-ray energy with sub-millimeter planar spatial resolution and sub-monolayer molecular sensitivity. We call this technique combinatorial NEXAFS.

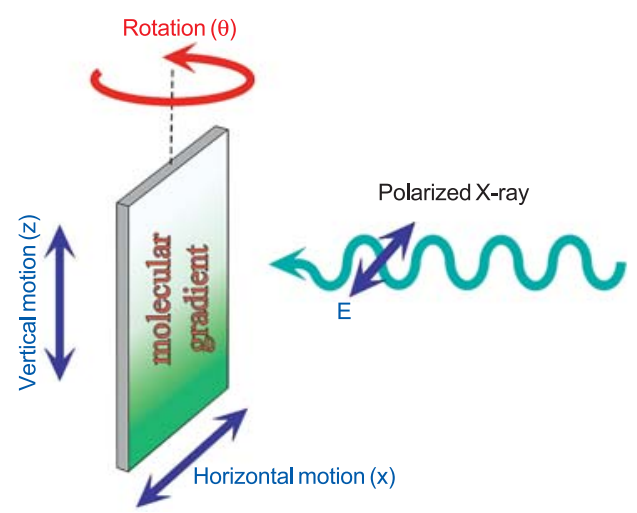

Figure 1: The principle of "combinatorial" NEXAFS.

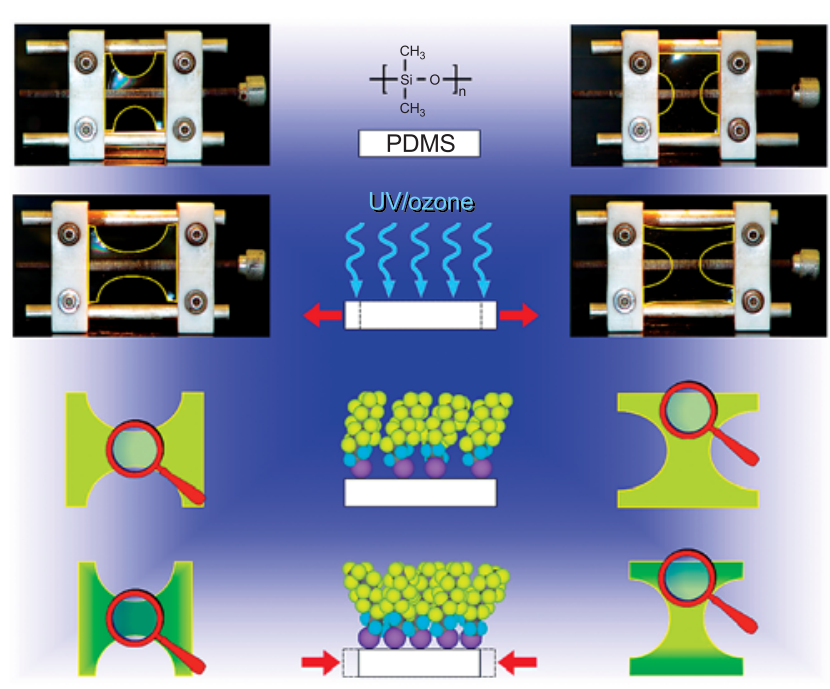

Figure 2: Schematic illustrating the process of creating molecular gradients on elastomeric substrates. The poly(dimethyl siloxane) (PDMS) network sheet is cut into "dog bone"-like shapes, stretched uniaxially and exposed briefly to ultraviolet/ozone treatment that produces surface-bound hydroxyl groups (PDMS-UVO). After exposing the substrate to a vapor of $t-F 8 H 2$, a uniform $t-F 8 H 2$ monolayer is generated on the surface PDMS-UVO. Upon releasing the strain from the substrate, a two-dimensional concentration gradient in $t-F 8 H 2$ is generated.

Combinatorial NEXAFS spectroscopy was used to map out the two-dimensional (2-D) in-plane concentration variation of $\mathrm{t}-\mathrm{F} 8 \mathrm{H} 2$ (Perfluorodecyltrichloro-silane), gradient-forming molecules. Such a 2-D gradient variant was produced by utilizing "mechanically assembled monolayers" (MAMs) technology on substrates in combination with in-plane asymmetric stretching of elastomeric substrates uniformly coated with organosilane precursors. Figure 2 summarizes the technological steps leading to the formation of 2-D gradients.

We first measured a fluorine K edge NEXAFS spectrum from a sample covered with a dense homogeneous monolayer of t-F8H2 on the stretched "dog bone" elastomeric substrate. The spectrum reveals the existence of two intense signals at $692.0 \mathrm{eV}$ and $694.5 \mathrm{eV}$, corresponding to the $1 \mathrm{~s}$ to $\sigma^{*} \mathrm{C}-\mathrm{F}$ excitations, respectively, superimposed on top of a broad fluorine excitation edge. The stretched "dog bone" substrates were then allowed to relax compacting the $\mathrm{t}-\mathrm{F} 8 \mathrm{H} 2$ homogenous monolayer in an asymmetric manner. We then performed the combinatorial NEXAFS experiments two-dimensionally by scanning the sample in the vertical and horizontal directions with $1 \mathrm{~mm}$ increments, at each step measuring the C-F $\sigma^{*}$ 
peak (chemical bond) intensity, as shown in Figure 3. The darker areas in the plot denote regions on the substrate with higher concentration of the C-F bond. The data in Figure 3 reveal that the concentration of C-F bonds on the sample varies spatially. Specifically, in sample S1, the concentration of C-F bonds is highest in the middle of the sample and decays towards the edges of the specimen. In sample S2, the edges of the sample contain a larger number of the $\mathrm{t}-\mathrm{F} 8 \mathrm{H} 2$ molecules, relative to the center. The ratio between the highest and lowest NEXAFS intensity signal in samples S1 and S2 was 1.35 and 1.26, respectively. The regions of the substrates with the highest C-F bond signal correspond to the locus of the maximum strain imposed after stretching the "dog bone" substrates in two orthogonal ways. Importantly, there is a gradual in-plane variation of the t-F8H2 concentration ranging between the high and low t-F8H2 density regions on the substrate. In order to correlate the results of the combinatorial NEXAFS C-F bond mapping experiments, the distribution of stresses in the S1 and S2 samples were calculated using a commercial software package. The results of the calculated stress distributions in the samples are shown as contour plots in Figure 3; strain increase is characterized by darker colors. Comparing visually the results in Figure 3, we conclude that the calculated results agree well qualitatively with the estimated strain distributions based on the sample contours and the concentration of the t-F8H2 molecules measured by combinatorial NEXAFS, respectively.
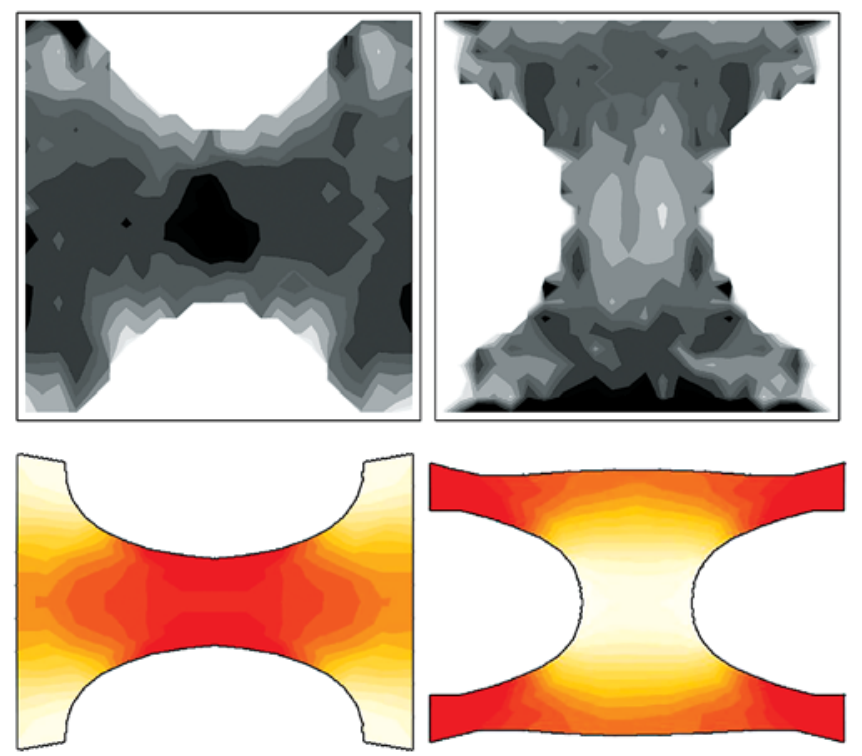

Figure 3: Top panel - C-F NEXAFS peak intensity maps, samples $S 1$ (left) and $S 2$ (right) after strain release. Darker regions indicate higher intensity of the $C-F$ signal. Bottom panel - Calculated strain maps in the stretched PDMS sheets corresponding to samples $S 1$ (left) and $S 2$ (right). The darker regions in the plots denote regions with larger strains.

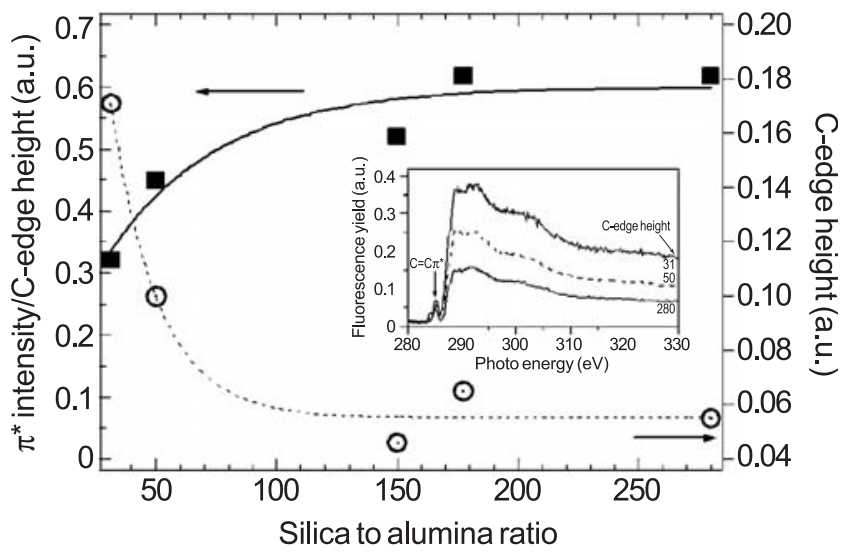

Figure 4: Inset shows a series of carbon K edge NEXAFS spectra for zeolite catalyst array (silica to alumina ratio of $31,50,280$ ). The carbon K edge height (dashed) and the rehybridized $1 s$ to $\pi^{*}$ peak intensity normalized by the carbon edge height (solid) versus the silica to alumina ratio of the zeolites.

We envision fluorescence yield combinatorial NEXAFS as a useful method for catalyst discovery, ultimately during an actual catalytic reaction. Catalyst discovery may be accomplished by searching for a desired chemical (i.e., spectral) change in a combinatorial NEXAFS bond rehybridization map of reaction intermediates formed on catalyst library arrays. In a prototypical catalyst discovery experiment, combinatorial NEAXFS was used to map the rehybridization of propylene reaction intermediates on zeolite catalyst arrays for measuring solid state acidity and activity. Figure 4 shows a series of carbon $k$ edge NEXAFS spectra for this catalyst array and the edge jump normalized intensity of the rehybridized $1 \mathrm{~s}$ to $\pi^{*} \mathrm{C}=\mathrm{C}$ peak. From the data, it appears that the chemical activity of the zeolite decreases rapidly as the silica to alumina ratio increases. Thus the combinatorial NEXAFS method allows the chemist to select the most acidic zeolite for this propylene reaction accelerating catalyst discovery.

\section{For More Information on this Topic}

D. Fischer, S. Sambasivan (Ceramics Division, NIST); J. Genzer, K. Efimenko, R. Bhat (NCSU)

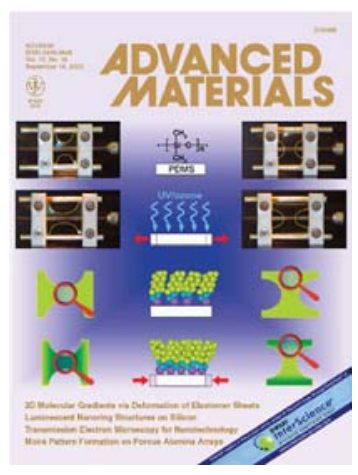

Materials, 15, 1545-1547 (2003). 


\section{Advances in X-ray Powder Diffraction Quantitative Analysis SRMs}

\begin{abstract}
Powder diffraction methods offer the direct means by which the components of crystalline mixtures may be quantified. While this method has been in use for decades, recent advances, particularly in the area of data analysis, have increased the accuracy of the technique to the point that the issue of the amorphous component has come to the fore. Requisite to addressing this measurement issue for the materials science community is the certification of NIST powder diffraction SRMs with regards to crystalline phase purity.
\end{abstract}

\section{Jim Cline}

$\mathrm{Q}$ uantitative analysis by powder diffraction methods is based on the fact that the diffraction intensity from a given phase is proportional to its concentration in the specimen. Data analysis strategies to quantify the concentrations may be considered in terms of two categories: the first consists of the "conventional," single peak methods that are based on the Reference Intensity Ratio, RIR, nomenclature; and the second is Quantitative Rietveld Analysis, QRA, wherein a reference diffraction pattern is computed using model functions for crystallographic, microstructural, and instrumental contributions. Parameters within these functions are then varied within a least-squares refinement to obtain an optimal fit between the computed and observed patterns. This method, first proposed for quantitative analyses in the mid-1980's, has raised the accuracy of the results to the extent that discussions of the amorphous content issue have become commonplace within the community.

Any finely divided solid possesses a large specific surface area that constitutes a boundary to the crystal structure. Due to relaxation and surface reactions, this region includes an amorphous layer of material that does not diffract like the bulk crystal. Its dimension, or mass fraction, will vary with the crystallography, chemistry, production history, and level of specific surface of the material. The amorphous fraction is, therefore, an innate characteristic of a powder that may well have a fundamental impact on its properties.

The diffraction experiment "sees" only the crystalline component of the specimen. If the experimentalist can account for all the crystalline phases in the unknown, then standardless analyses of the crystalline components of the mixture are possible. The results, however, will be reflective only of the crystalline components in the specimen and may not assess the mass fraction of the powder components of the mixture accurately due to the presence of amorphous layers. However, analysis of the nondiffracting amorphous component is possible when an internal standard is admixed with the specimen. The amorphous component is then determined from the discrepancy between the mass fraction obtained from the diffraction experiment, and that of the weighing operation. Requisite to such analyses are: 1) accurate measurement methods and 2) standards that have been characterized with respect to their amorphous phase fraction.

The measurement accuracy and amorphous content issues are inextricably linked; one cannot assess the accuracy of a quantitative measurement with a comparison to a weighing operation without some a priori knowledge of the amorphous component. This not being plausible, we construct an experimental design utilizing two phase mixtures wherein the amorphous component of one phase, the reference phase, is varied in a systematic manner. With this approach, results are based on the accurate determination of the Bragg scattering intensity from the two phases, as opposed to the weak diffuse scattering for the amorphous component itself. The design must also embody the means to verify results through both internal and external consistency checks.

Table 1: Microstructure data from the five lots of silicon and SRM 640c $\left(d_{L}=\right.$ particle size by laser light scattering, $\sigma=B E T$ specific surface area, and $D_{x}=$ TOF extinction diameter)

\begin{tabular}{ccccccc} 
Lot & $\mathbf{1}$ & $\mathbf{6 4 0 c}$ & $\mathbf{2}$ & $\mathbf{3}$ & $\mathbf{4}$ & $\mathbf{5}$ \\
\hline$d_{\mathrm{L}}, \mu \mathrm{m}$ & 3.47 & 3.51 & 10.03 & 15.07 & 19.47 & 24.14 \\
$\sigma, \mathrm{m}^{2} / \mathrm{g}$ & 1.728 & 1.402 & 0.703 & 0.424 & 0.335 & 0.270 \\
$D_{\mathrm{x}}, \mu \mathrm{m}$ & - & 3.7 & 5.6 & 6.5 & 7.6 & 8.1 \\
\hline
\end{tabular}

The primary NIST SRM for quantitative analysis via powder diffraction is SRM 676, an alumina powder originally certified in 1992. SRM 676 was used as the base material in the present work, while the reference phase was the intrinsic, float zone silicon used for the feedstock of SRM 640c. SRM 640c has the highest purity and the lowest defect concentration of any crystalline silicon available. Implicit in our interpretation of the data is that the silicon has no crystallographic or microstructural defects, that all amorphous material associated with it is 
confined to the crystallite surface, and that the amorphous layer thickness is invariant with respect to crystallite size. Five lots of silicon were prepared and characterized; microstructural data for these and SRM 640c are shown in Table 1. A series of 50-50 mass fraction mixtures of silicon and SRM 676 were prepared. The mass fraction of silicon, determined from the diffraction experiments was then plotted relative to the surface area, or amorphous content, of the silicon. These data were then extrapolated to yield a value for mass fraction of silicon that would possess "zero" amorphous content, thereby yielding the mass fraction of alumina that would be determined using an imaginary silicon sample of $100 \%$ crystalline content. Thus, the discrepancy between the mass fraction measured via diffraction and that determined from the initial weighing indicated the true amorphous content of the alumina of SRM 676.

The powder diffraction methods used for this study were chosen for their ability to overcome the factors that typically confound the accurate measurement of Bragg scattering intensity. Crucial to the success of the experiment was the need to address the effects of extinction that would, in particular, affect the scattering intensity from the coarse grained silicon. Extinction can only be considered with dynamical diffraction theory as a result of destructive interference in the standing waves within the crystal during the diffraction condition. Generally, $R=Q \mathrm{f}(\mathrm{A})$, where $R$ is the diffraction intensity, $Q$ is the diffraction intensity per unit volume, and $\mathrm{f}(\mathrm{A})$ is a function of diffraction geometry:

$$
A=\left(e^{2} \lambda F t\right) /\left(m c^{2} V\right)
$$

where $\lambda$ is the wavelength, $F$ is the Structure Factor, $t$ is the crystallite size, and $V$ is the volume of the unit cell. One can see that the parameter which may be varied to address extinction effects is the radiation wavelength.

Two data collection methods were selected, largely in the context of the radiation wavelengths that they employed. The neutron time-of-flight, TOF, powder diffraction method, using radiation that is multi-wavelength in nature, permits a robust analysis of the extinction domain size, and its correction, using the Sabine model within the Rietveld analysis of the raw data. The second approach is to reduce $\lambda$ to the point where the extinction effect is inoperative. The $56 \mathrm{keV}$ energy of the x-ray radiation used for the second set of powder diffraction experiments met this condition. Convergence of these two data collection and analysis strategies ensures credibility of the final result.

The Rietveld analyses of the data were performed in a single refinement of the suite of data from each method. This approach permitted parameters to be constrained in a physically valid manner consistent with crystallography and microstructure of the specimens. The TOF data included extinction domain size parameters that are shown in Table 1. The phase fractions of the silicon are plotted relative to its surface area in Figures 1 and 2. One can observe in these data: 1) the expected decrease in the phase fraction of silicon as its surface area increases and 2) very good correlation between the two methods. Results of a linear least-squares fit to the data shown in the figures indicate that the $y$ intercept values for these two independent measurements are within acceptable error bounds.

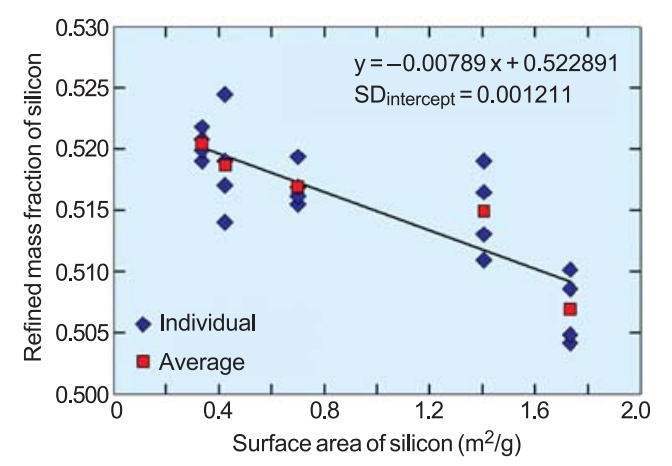

Figure 1: QRA of Si/SRM 676 data from TOF powder diffraction.

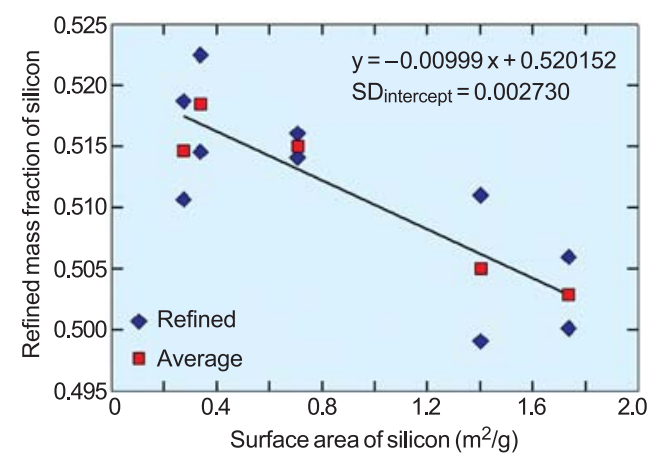

Figure 2: QRA of Si/SRM 676 data from high energy $x$-ray powder diffraction.

The analysis of the y intercept leads to a certified value of $8.25 \% \pm 1.52 \%$ for the amorphous content of SRM 676. Because the suite of NIST powder diffraction quantitative analysis SRMs were certified relative to SRM 676, they now can be certified for phase purity.

The amorphous value determined for SRM 676 is a surprisingly large value, thought, for this alumina powder, to be due to transition alumina impurities that were not fully decomposed upon thermal processing during manufacture. This value indicates that many powders that offer "clean" diffraction patterns, with no discernable diffuse scattering in the background, may actually be $\approx 95 \%$ pure. Use of the suite of NIST SRMs resolves this issue and provides the diffraction community with the means of determining the amorphous layer thickness of powders, a previously inaccessible characteristic.

\section{For More Information on this Topic}

NIST Standard Reference Materials, http://ts.nist.gov/ts/htdocs/230/232/232.htm. 


\section{Advanced Manufacturing Processes}

The competitiveness of U.S. manufacturers depends on their ability to create new product concepts and to speed the translation from concept to market while decreasing product cost. This is equally true for well-established "commodity" industries, such as automotive and aerospace, and rapidly growing or emerging industries, such as biotechnology and nanotechnology. For existing products, manufacturing is a critical step in reducing product cycle time. Rapid, low-cost development of manufacturing processes is needed to incorporate new materials into complex product shapes with higher performance at equivalent or lower cost as the competing, established materials and methods. For innovative product concepts, new materials with increasing functionality are needed to translate these concepts to reality.

To realize such improvements in materials and manufacturing, MSEL is developing robust measurement methods, models, standards, and materials and process data needed for design, monitoring, and control of manufacturing processes. A growing challenge is being able to design, monitor, and control such materials and manufacturing processes at size scales from nanometers to meters. The Advanced Manufacturing Processes Program focuses on the following high-impact areas:

- Combinatorial, high-throughput methods for materials ranging from thin films and nanocomposites to micro- and macroscale material structures;

- Industry-targeted R\&D centered on unique measurement facilities in forming of lightweight metals for automotive applications, polymer processing, and high-speed machining;

- Innovative testbeds for emerging materials, including carbon nanotubes and fuel cells;

- National traceable standards having a major impact on trade, such as hardness standards for metals and process standards for polymers; and

- Innovative, physics-based process modeling tools.

Our research is often conducted in close collaboration with industrial consortia and standards organizations. These collaborations not only ensure the relevance of our research, but also promote rapid transfer of our research to industry for implementation. Three projects focused on Advanced Manufacturing Processes are highlighted below.

\section{NIST Combinatorial Methods Center (NCMC)}

The NCMC develops novel high-throughput measurement techniques and combinatorial experimental strategies specifically geared towards materials research.
These tools enable rapid acquisition and analysis of physical and chemical data, thereby accelerating the pace of materials discovery and knowledge generation. By providing measurement infrastructure, standards, and protocols, and expanding existing capabilities relevant to combinatorial approaches, the NCMC lowers barriers to the widespread industrial implementation of this new $\mathrm{R} \& \mathrm{D}$ paradigm. MSEL uses a two-pronged strategy for accelerating the development and implementation of these approaches: an active intramural $\mathrm{R} \& \mathrm{D}$ program that demonstrates the ability of combinatorial methods to produce cutting-edge scientific research and an ambitious outreach activity; key to this effort is the validation of these approaches with respect to traditional "one at a time" experimental strategies.

\section{Forming of Lightweight Metals}

Automobile manufacturing is a materials intensive industry that involves about $10 \%$ of the U.S. workforce. In spite of the use of the most advanced, cost-effective technologies, this globally competitive industry has major productivity issues related to measurement science and data. Chief among these is the difficulty of designing stamping dies for sheet metal forming. An ATP-sponsored workshop ("The Road Ahead," June 20-22, 2000) identified production of working die sets as the main obstacle to reducing the time between accepting a new design and actual production of parts. This is also the largest single cost (besides labor) in car production. To benefit from weight savings enabled by new high-strength steels and aluminum alloys, a whole new level of formability measurement methods, models, and data is needed, together with a better understanding of the physics behind metal deformation. MSEL is working with U.S. automakers and their suppliers to fill this need.

\section{Polymer Processing}

Polymers have become ubiquitous in the modern economy because of their processability, high functionality, and low cost. However, these materials can exhibit complex and sometimes catastrophic responses to the forces imposed during manufacturing, thereby limiting processing rates and the ability to predict ultimate properties. The focus of our polymer research is on microfluidics and microscale processing, modeling of processing instabilities, and on-line process monitoring of polymers. Our unique extrusion visualization facility combines in-line microscopy and light scattering for the study of polymer blends, extrusion instabilities, and the action of additives. These measurements are carried out in close collaboration with interested industrial partners.

Contact: Carol A. Handwerker (Metallurgy Division), Andrew J. Allen (Ceramics Division) 


\section{Synchrotron X-ray Measurements in Support of Solid Oxide Fuel Cell Development}

\begin{abstract}
Control of solid oxide fuel cell microstructure and electrochemistry close to the electrode-electrolyteinterconnect interfaces is important for optimizing fuel cell performance and lifetime. Quantifying the associated gradients and degradation effects during service life is needed to exploit fully the underlying phenomena governing the material properties. A combination of third-generation $x$-ray synchrotron measurements at the Advanced Photon Source seeks to address this need.
\end{abstract}

\section{Andrew J. Allen}

Colid oxide fuel cell (SOFC) systems are undergoing $\checkmark$ rapid development for large-scale power applications. We are addressing the long-term need to improve the control and integrity of the gradient microstructures within the cathode and anode close to, and at, the interfaces with the dense electrolyte layer. At the typical SOFC operating temperatures of $600{ }^{\circ} \mathrm{C}$ to $800{ }^{\circ} \mathrm{C}$, this issue can influence the in-service performance degradation and SOFC lifetime during successive thermal cycles. The operative electrochemistry at the electrolyte-electrode interfaces is, itself, ultimately affected by these changes. A combination of third-generation x-ray synchrotron measurements at the Advanced Photon Source seeks to quantify the microstructure and electrochemistry, and establish the relationships between them.

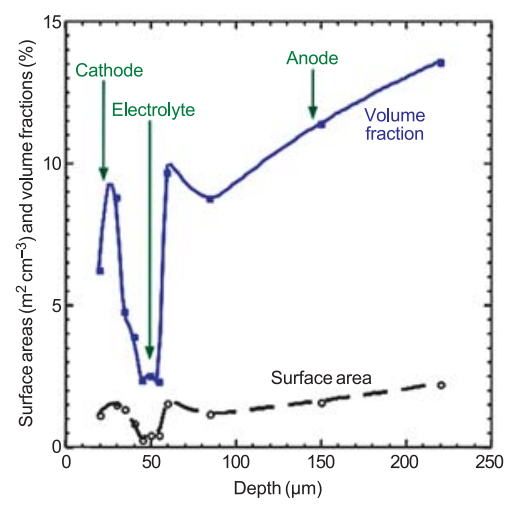

Figure 1: USAXS.

Figure 1 presents spatial variations in the absolute void volume fraction and surface area within a SOFC, with respect to the electrolyte membrane position. The data were obtained by ultrasmall-angle $x$-ray scattering (USAXS), as were complete void size distributions. A dense yttria-stabilized zirconia (YSZ) electrolyte layer clearly separates the porous cathode (lanthanum strontium manganate or LSM) and anode (Ni/YSZ cermet) layers. An $x$-ray vertical beam dimension of less than $10 \mu \mathrm{m}$ was achieved to provide the needed spatial resolution. Degradation or changes in the measured void variations, as a function of fabrication process or service cycling, would affect both the SOFC electrochemical performance and structural integrity.

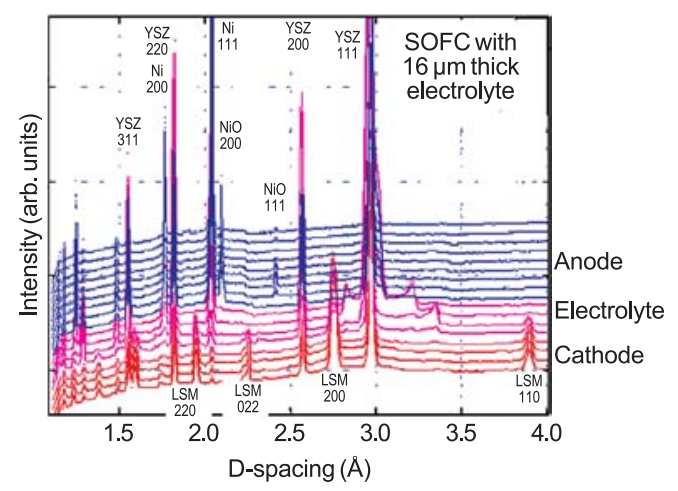

Figure 2: $H E-W A X S$.

Figure 2 shows high-energy wide angle x-ray scattering (HE-WAXS) measurements of the complete $x$-ray diffraction pattern at successive positions with respect to the SOFC electrolyte layer. HE-WAXS and corresponding high-energy small-angle x-ray scattering (HE-SAXS) studies of the void surface area variation (calibrated by cross-reference to USAXS) have been made with a $5 \mu \mathrm{m}$ $\mathrm{x}$-ray beam size. At the $80 \mathrm{keV}$ x-ray energy, both WAXS and SAXS data can be obtained with similar measurement geometries and at identical sample positions. Thus, the spatial variations in void microstructure and phase can be cross-referenced to infer the electrochemical behavior of the electrode microstructures close to the interface with the YSZ electrolyte.

Recently, the electrochemical issues have been explored more directly through measurement of x-ray fluorescent yield (FY) spectra. We have used an in-situ heating chamber to study SOFC cathode layers under controlled atmosphere conditions at temperatures close to those encountered in an operating SOFC. By combining the electrochemical data obtained for specific interfaces with knowledge of the SOFC microstructure and phase information above, we seek to facilitate improved SOFC design.

\section{Contributors and Collaborators}

J. Woicik (Ceramics Division, NIST); J. Ilavsky, J. Almer (Argonne National Laboratory); A. Virkar (University of Utah); L. Wilson (NETL); P. Singh, J. Stevenson (PNNL); J. Ruud (GE Corporate Research); M. Seabaugh (NexTech Materials) 


\section{Nanometrology}

The burgeoning field of nanomaterials extends across the full range of traditional material classes, including all forms of metals, polymers, and ceramics. No previous materials technology has shown so prodigiously a potential for concurrent advances in research and industry as does the field of nanomaterials in mechanical devices, electronic, magnetic, and optical components, quantum computing, tissue engineering and other biotechnologies, and as-yet unanticipated exploitations of as-yet undiscovered novel properties of nanoscale assemblies of particles. Already, there is growing excitement surrounding the ability of some molecules or particles to self-assemble at the nanoscale to form new materials with unusual properties. Nanometrology, i.e., the ability to conduct measurements at these dimensions, to characterize the materials, and to elucidate the structure and nature of these new and novel assemblies, is a requisite and fundamental cornerstone that must be established securely if this technology is to flourish.

NIST is uniquely positioned to lead the development of the measurement methods, instrumentation, standards, and reference materials that, together, will form the metrological infrastructure essential to the success of nanotechnology.

The MSEL Nanometrology Program incorporates basic measurement metrologies to determine material properties, process monitoring at the nanoscale, nanomanufacturing and fabrication techniques, and structural characterization and analysis techniques such as advanced imaging and multiscale modeling. The Program comprises 22 projects in the Ceramics, Materials Reliability, Metallurgy, and Polymers Divisions, and includes structural characterization using neutron scattering at the NIST Center for Neutron Research (NCNR). The projects cover a wide range of measurement and characterization methods grouped into the areas of mechanical property measurement, chemical and structural characterization and imaging, fabrication and monitoring of nanoprocesses and events, and modeling of nanoscale properties. In each area, we work to advance basic measurement capabilities and lead the intercomparison, standardization, and calibration of test methods. The newly completed Advanced Measurement Laboratory at the NIST Gaithersburg site provides an incomparable environment for accurate nanoscale metrology.

In the area of mechanical property measurement, we are developing and standardizing techniques for determining nanoscale elastic properties (elastic moduli, Poison's ratio, and internal stress), plastic deformation, density, adhesion, friction, stiction, and tribological behavior. Work in nanoindentation, used extensively in determining mechanical properties of thin films and nanostructures, focuses on developing traceable calibration methodologies and standard test methods. We also use atomic force acoustic microscopy, surface acoustic wave spectroscopy, and Brillouin light scattering to measure the mechanical properties of thin films. In addition, we are developing micro- and nano-scale structures and test methods to measure strength and fracture behavior of interfaces and materials having very small volumes.

The chemical and structural characterization and imaging utilize neutron and $\mathrm{x}$-ray beam lines at three facilities: the NCNR; the National Synchrotron Light Source at Brookhaven National Laboratory; and the Advanced Photon Source at Argonne National Laboratory. Innovative scattering and spectroscopy methods are advancing our ability to obtain a wide range of chemical and structural information at the nanoscale, including chemical bond identification and orientation, polyelectrolyte dynamics, and equilibrium structures. In collaboration with three other NIST laboratories, we are developing electron microscopy and spectroscopy instrumentation for quantitative, $3 \mathrm{D}$ chemical imaging at the nanoscale. Other characterization projects include work on gradient reference specimens for the calibration of advanced scanning probe microscopy, and the application of carbon nanotubes as physical probes of cell membranes.

Efforts in the fabrication and monitoring of nanoscale processes and events include the study of electrochemical and microfluidic methods for fabricating nanostructures, novel approaches to nanocalorimetry for the study of interfacial reactions, in situ observations of nanoparticle and nanotube dispersion and alignment, and advanced instrumentation for nanotribology experiments.

Finally, we have extensive efforts in the theory, modeling, and prediction of material properties and behavior extending from nanoscale to macroscale dimensions. Modeling efforts include large-scale finite element methods, multiscale Green's function methods, classical atomistic simulations, first principles, and quantum mechanical calculations using density functional theory. Often, several modeling methods must be combined into one study to accurately describe the material behavior; thus, we pay great attention to the correct interfacing between models operating at different length scales, to ensure that our models properly capture the physics of both components and total systems.

Contact: Douglas T. Smith, Stephen M. Hsu (Ceramics Division) 


\section{Chemistry and Structure of Nanomaterials}

Successful nanoscale materials fabrication is empowered by a detailed knowledge of the chemistry and structure of surface bound molecules; e.g., the optimization of self-assembled monolayers, molecular templates, micro-electro-mechanical system lubricants, and functionalized nanotubes. Near-Edge X-ray Absorption Fine Structure (NEXAFS) spectroscopy is ideally suited to measure non-destructively chemical bond concentration, rehybrization, and orientation with sub-monolayer molecular sensitivity in diverse nanoscale materials. Furthermore, NEXAFS can distinguish chemical bonding in the light elements, measure the orientation of interfacial molecules, and separately measure surface versus bulk chemistry simultaneously.

\section{Daniel A. Fischer}

$\mathrm{M}$ aterials having low energy surfaces are used in many applications, for example, in non-wetting surfaces or fouling resistant marine coatings. We have produced a photo-responsive polymer surface by combining the reversible photo-switching nature of azobenzene with the self-assembly nature and low surface energy properties of semi-fluorinated segments, to create a fluoroazobenzene molecule surface. Upon UV exposure, this surface reorients between hydrophobic and less hydrophobic states, as shown in Figure 1 (left upper and lower panels). For such surfaces, one could imagine applications ranging from low cost surface patterning to polymer surfaces that would adsorb biological macromolecules on cue.

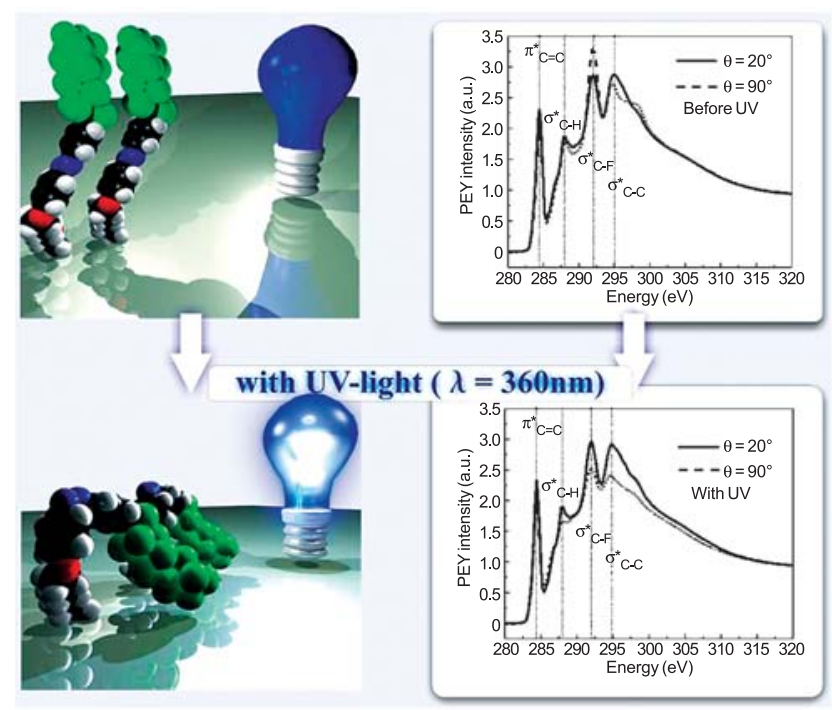

Figure 1: UV light reorients fluorobenzene semi-fluorinated segments (green) downwards, i.e., to a less hydrophobic state.
We have utilized NEXAFS to observe, verify, and quantify the reversible cis-trans molecular conformation transformation from hydrophobic to less hydrophobic states. The right panels of Figure 1 (upper and lower) show polarization dependent NEXAFS anisotropy behavior of the $\mathrm{C}-\mathrm{F}$ and $\mathrm{C}-\mathrm{C}$ peaks which reverse with in situ UV light exposure highlighting the reorientation of the semi-fluorinated segments.

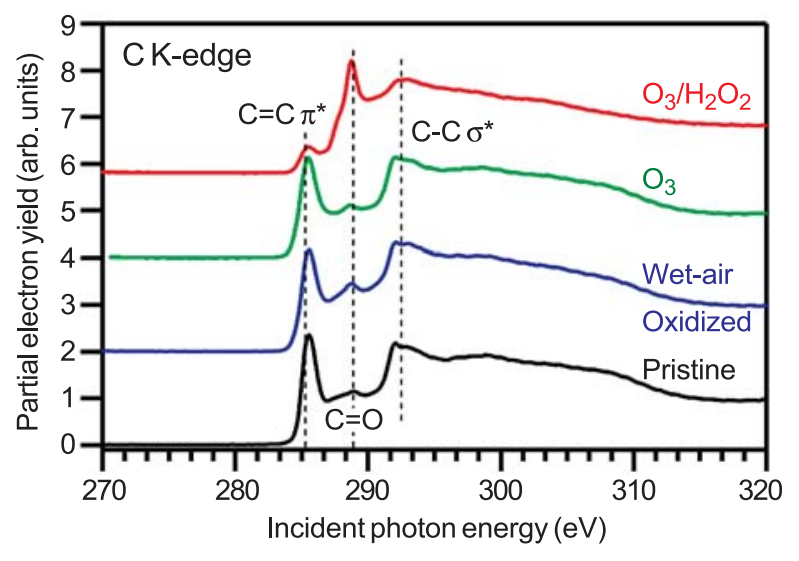

Figure 2: Carbon NEXAFS of oxidized/functionalized nanotubes.

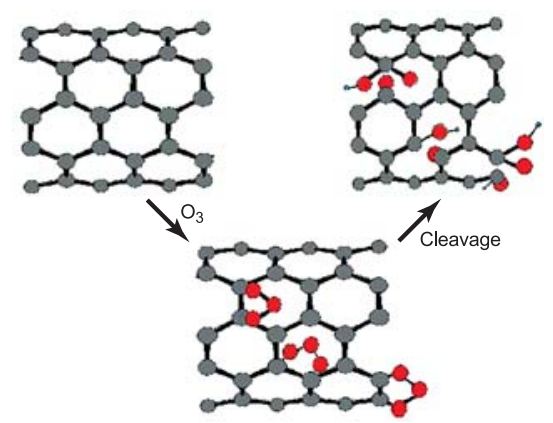

Figure 3: Model of peroxide functionalization of nanotubes.

Application of NEXAFS spectroscopy to the study of electronic structure and chemical composition is illustrated in Figure 2 for various chemically functionalized, singlewalled, carbon nanotubes. Upon peroxide functionalization, the $\mathrm{C}=\mathrm{C}$ ring resonance is greatly diminished on extensive sidewall functionalization indicating loss of extended conjugation and disruption of nanotube electronic structure. The $\mathrm{C}=\mathrm{O}$ peak intensity is greatest for peroxide chemistry. NEXAFS spectroscopy supports a model of peroxide funtionalization, shown in Figure 3.

\section{Contributors and Collaborators}

S. Samabasivan (Ceramics Division, NIST); X. Li, C.K. Ober (Cornell); A. Hexemer, E.J. Kramer (UCSB); S. Banerjee, T. Benny, S.S. Wong (SUSB); J.A. Misewich (BNL) 


\section{Thermochemistry and Metrology of Interfacial Interactions}

\begin{abstract}
Reactions occurring at interfaces during the high-temperature processing of complex electronic materials involve diverse chemical compositions. To optimize the design and control of these interfaces, we are developing a generic thermodynamic/kinetic model for interfacial interactions. The model includes modification of bulk reaction and mass transport parameters to accommodate nanoscale observations.
\end{abstract}

\section{Lawrence P. Cook, Winnie Wong-Ng, and Igor Levin}

State-of-the-art electronic devices contain complex materials with chemical constituents ranging widely over the periodic table; furthermore, new materials are continually being added. Many electronic packaging materials consist of metals, ceramics, and semiconductors, with bi-phasic interfaces of several types. Processing temperatures may extend up to $900{ }^{\circ} \mathrm{C}$ where diffusion and reaction can be significant. Two principal problems arise in the treatment of such interactions: 1) the application of equilibrium thermodynamic data to the non-equilibrium growth of interfacial reaction zones, and 2) the transition from micro-scale (bulk dominated), to nanoscale (surface dominated) phenomena. To address these issues, we have instituted a combined thermodynamic/kinetic approach to investigate the model system $\mathrm{Ag}-\mathrm{Bi}_{2} \mathrm{O}_{3}-\mathrm{Nb}_{2} \mathrm{O}_{5}$-O, as outlined in Figure 1.

\section{Ag- $-\mathrm{Bi}_{2} \mathrm{O}_{3}-\mathrm{Nb}_{2} \mathrm{O}_{5}-\mathrm{O}$ Model System}

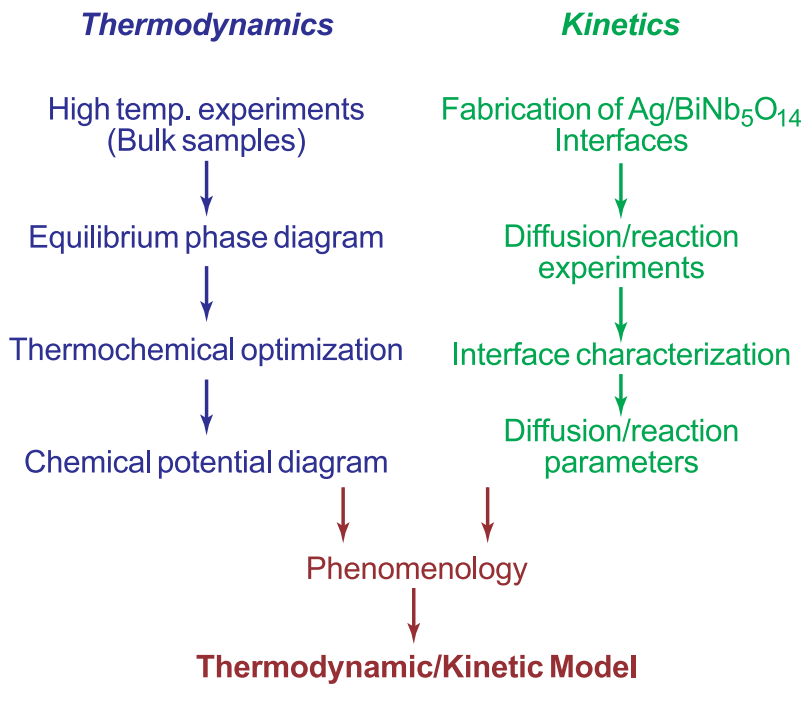

Figure 1: Flow chart for development of interfacial model based on $\mathrm{Ag}-\mathrm{Bi}_{2} \mathrm{O}_{3}-\mathrm{Nb}_{2} \mathrm{O}_{5^{-}} \mathrm{O}$.

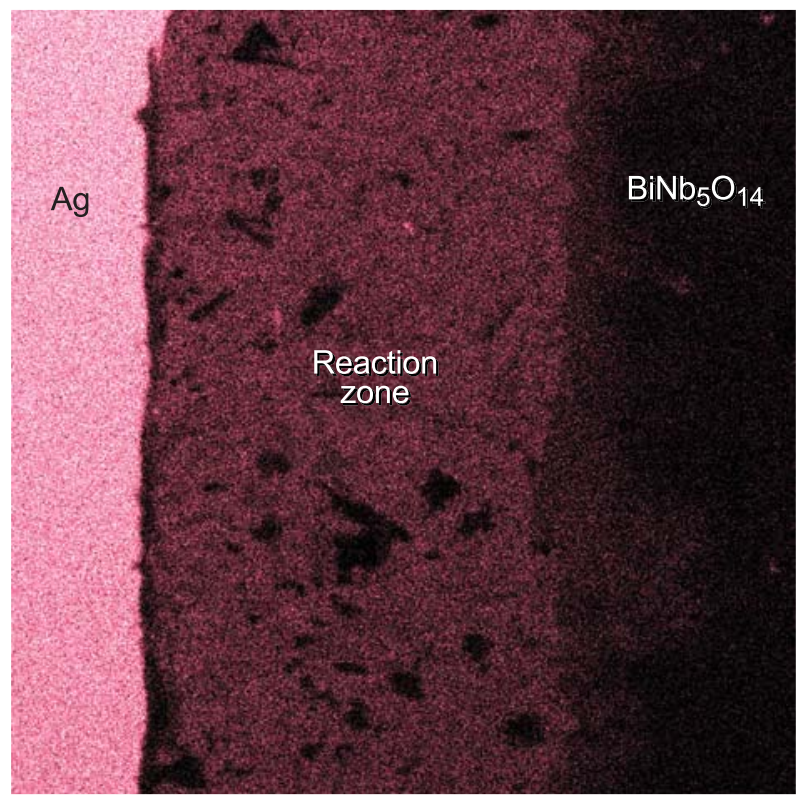

Figure 2: $\mathrm{Ag} \mathrm{L} \mathrm{L}_{\alpha} \mathrm{X}$-ray map of $\mathrm{Ag} / \mathrm{BiNb}_{5} \mathrm{O}_{14}$ reaction zone $\left(\approx 80 \mu \mathrm{m}\right.$ wide) produced by annealing in air for $10 \mathrm{~h}$ at $850^{\circ} \mathrm{C}$, with an applied uniaxial compression of $\approx 1 \mathrm{MPa}$.

Progress includes determination of a preliminary phase diagram at $850^{\circ} \mathrm{C}$, and development of a provisional thermodynamic model. Subsequent construction of a chemical potential diagram has allowed us to predict alternative diffusion and reaction paths. Observations on $\mathrm{Ag} / \mathrm{BiNb}_{5} \mathrm{O}_{14}$ reaction couples (Figure 2) confirm the relatively high thermodynamic mobility of Ag. Thermogravimetric data on the interfacial reaction require more than a simple parabolic rate law, due to the rapid initial spread of Ag along reaction site surfaces. By comparison with particulate interfacial systems, a two-step reaction model is proposed, for which rate constants can be obtained.

Data for the model system are of direct interest to the electronics community because $\mathrm{Ag}$ is an important metallization component in many dielectric ceramics. It is anticipated that continued work on $\mathrm{Ag}-\mathrm{Bi}_{2} \mathrm{O}_{3}-\mathrm{Nb}_{2} \mathrm{O}_{5}-\mathrm{O}$ will lead to the goal of a comprehensive thermodynamic/kinetic model. The model will be tested, iteratively refined, and extended to other systems.

\section{Contributors and Collaborators}

M.D. Vaudin, P.K. Schenck, T. Vanderah, M. Green (Ceramics Division, NIST); W. Luecke (Metallurgy Division, NIST); C. Randall, M. Lanagan (Center for Dielectric Studies, Pennsylvania State University) 


\section{Particle Metrology and Nanoassembly}

As technology migrates toward smaller physical dimensions, new analytical approaches are required to characterize material properties and to investigate critical issues. Our primary focus is the application of metrology and the development of new methods and standards for measuring the physical and surface properties of nanostructured particle systems. Applications include functional materials and devices for catalysis, power generation, and microelectronic, pharmaceutical, and biotechnology industries.

\section{Vince Hackley}

$\mathrm{N}$ anocrystalline oxides of alkaline-earth cations produced by a supercritical drying process exhibit unique and highly reactive surface chemistries. As a result, these materials have been studied extensively as destructive adsorbents, catalysts, and bioactive agents. Investigations were concluded regarding the role of cation size on the evolution of microstructure in these materials and on the dispersion properties in aqueous $\mathrm{NaCl}$ solution. A series of small-angle neutron scattering (SANS) experiments were performed at the NIST reactor in collaboration with researchers at Kansas State University. These results indicate a complex picture for structure formation during the drying and annealing processes, with string-like gel morphology giving way to fractally rough particulate assemblies of compacted nanocrystals.

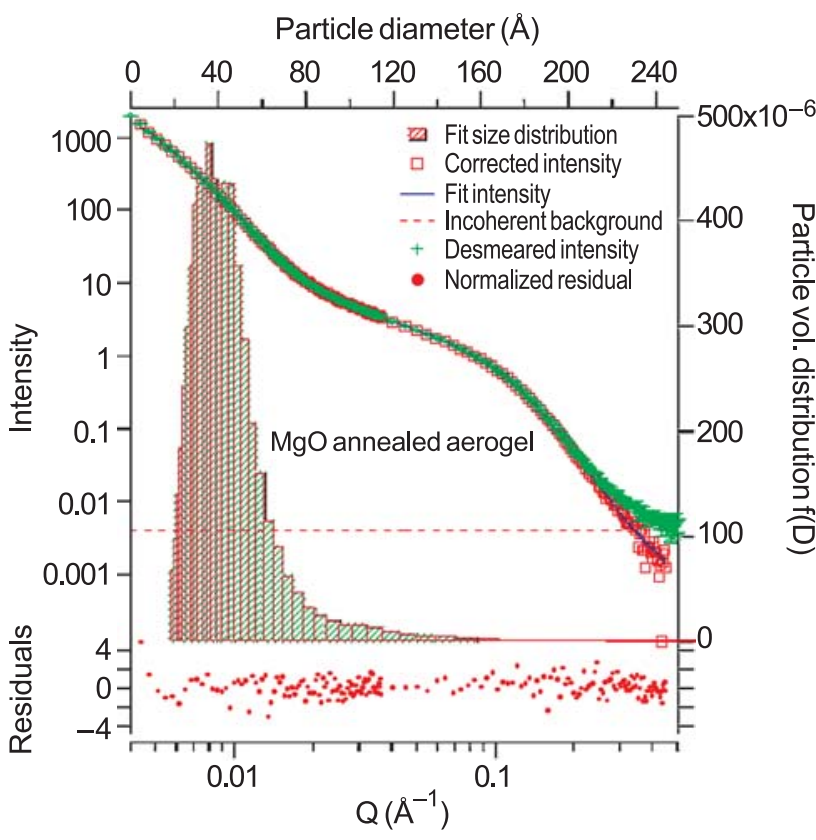

Figure 1: Maximum entropy fit to SANS data for annealed MgO aerogel.
Data were analyzed using the Unified Model of Beaucage and the maximum entropy method (see Figure 1). The scale of the finest structural features increases with increasing cation size for the annealed product. Analysis indicates the absence of mass fractal structures but the presence of surface fractal-like objects. A broad correlation peak in the data for heat-treated $\mathrm{Mg}$ and $\mathrm{Ca}$ oxides gives evidence for some local ordering of the nanocrystals. This data will help provide a more complete understanding of the structural development in this complex and technologically important system.

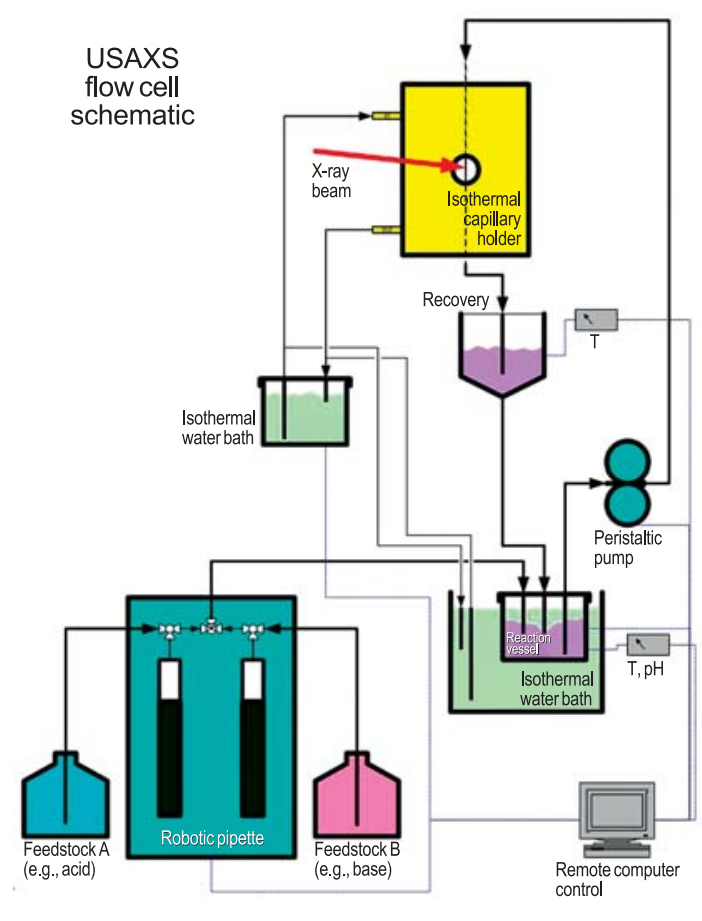

Figure 2: Schematic diagram of capillary flow cell.

A capillary flow-cell (Figure 2) was developed and commissioned for the ultra-small-angle $\mathrm{x}$-ray scattering (USAXS) instrument on the UNICAT beam line at the Advanced Photon Source. This new capability will permit in situ investigations of complex multiphase particulate systems under controlled flow conditions. Initial applications include the dispersion of single-wall carbon nanotubes in collaboration with Rice University and depletion effects in binary colloidal suspensions.

\section{Contributors and Collaborators}

A. Allen, L. Lum (Ceramics Division, NIST); D. Ho (NIST Center for Neutron Research); K. Klabunde (Kansas State University); M. Pasquali (Rice University); P. Jemian (University of Illinois) 


\section{Mechanical Metrology for Small-Scale Structures}

\begin{abstract}
Myriad industrial and biological systems are composed of small-scale structures for which the mechanical behavior is not accurately known. Optimizing the performance and reliability of these systems requires either mechanical property measurements on specimens of these structures harvested from the appropriate phases or interfaces of the system, or the ability to test these structures in situ. We are developing standardized testing configurations and methodologies for localized measurements of strength and fracture toughness of materials and interfaces at the micro- to nanometer-length scale.
\end{abstract}

\section{Edwin R. Fuller, Jr. and George D. Quinn}

$\mathrm{T}_{\mathrm{s}}$ his project aims to: (1) measure mechanical properties of microstructures for myriad industrial and biological systems that cannot be fabricated in bulk samples; (2) study small-scale phenomena that may be controlled by surface effects, e.g., the influence of surface stresses on crack nucleation and extension; and (3) obtain quantitative mechanical property data of materials and interfaces for designing small-scale structures and components and for assessing their mechanical reliability. To address these goals, well-characterized testing configurations must be developed for small-scale measurements of strength and crack extension. We are pursuing four tasks: (1) configuration design and finite element analysis; (2) specimen fabrication; (3) mechanical testing and fracture analysis (fractography); and (4) length and force metrology. Work in the Ceramics Division this year has focused on the first and third areas. Two collaborations were established in the fabrication task: one with James A. Beall of the Quantum Electrical Metrology Division (817) in NIST Boulder, and one with Northwestern University. Work in the fourth task will

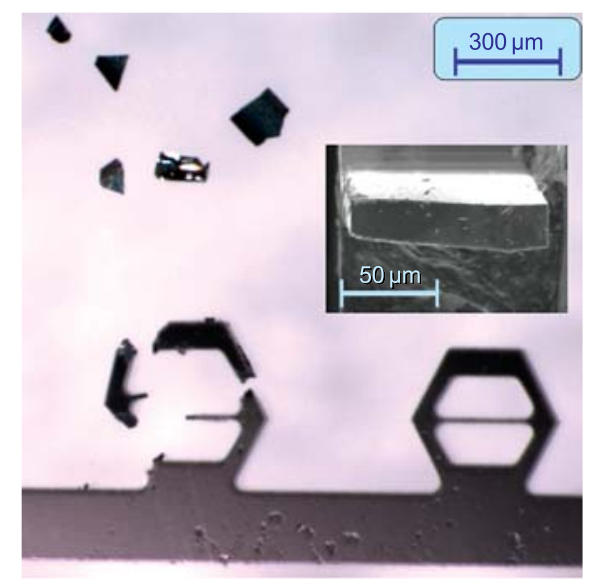

Figure 1: Prototype specimen design. come in subsequent years, most likely in collaboration with the Manufacturing Engineering Laboratory. Significant progress has been made in the design of a compressively loaded test configuration with a welldefined, tensile gage section. Such a specimen can be loaded using a depth-sensing nanoindenter as a universal testing machine, thereby giving a record of both applied load and load-point displacement. One of these specimens, fabricated by James Beall from a silicon wafer by deep reactive ion etching, is shown in the right-side of Figure 1. The configuration is similar to a theta specimen, except that the geometry is hexagonal. When a load (per unit thickness) is applied to the top beam, a uniform uniaxial tensile stress results in the middle gage section. Finite element analysis gives (horizontal) gage section stresses on the order of $1.25 \mathrm{GPa}$ for $50 \mathrm{mN} / \mu \mathrm{m}$ of applied load. For a $2 \mathrm{~N}$ applied load, these $100 \mathrm{~mm}$-thick specimens generate $500 \mathrm{MPa}$ of tensile stress in the gage section. The left-side of Figure 1 shows a reconstructed failed specimen. The insert shows the fracture surface of the gage section, and the two [111] cleavage facets that were formed. Alternate geometries, including a round theta specimen, are also being considered.

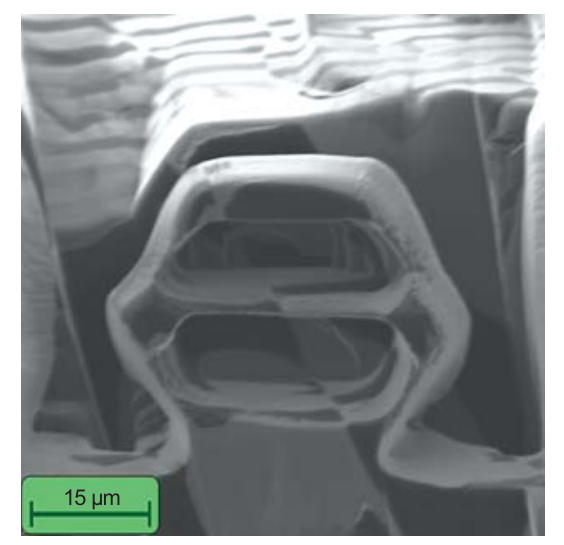

Figure 2: Hexagonal theta specimen by FIBing.

To extend this technique to a wide variety of materials and systems, general fabrication procedures need to be developed. Towards this objective, focusedion-beam (FIB) milling is being explored in collaboration with Northwestern University. Figure 2 shows our first attempt at producing a hexagonal theta specimen by FIBing. It has been scaled-down by about a factor of 10 , and is fabricated from a lamellar directionally solidified eutectic of $\mathrm{Ni}_{0.5} \mathrm{Co}_{0.5} \mathrm{O}$ and $\mathrm{ZrO}_{2}$.

\section{Contributors and Collaborators}

D. Xiang, D.T. Smith (Ceramics Division, NIST); A. Jillavenkatesa (Standards Services Division, NIST); J.A. Beall (Quantum Electrical Metrology Division, NIST); N. Alem, V.P. Dravid (Northwestern University) 


\section{Nanoindentation Methods and Standards}

Nanoindentation has rapidly become the method of choice for quantitative determination of mechanical properties of thin films and small volumes of material, but standardization efforts have lagged far

behind the application of the technique in industrial research and development. Over 1000 commercial nanoindentation instruments from a variety of manufacturers are currently in use, with no traceable force calibration and an extremely limited choice of standardized test methods. We work with standards groups and National Measurement Institutes around the world to develop standard reference materials (SRMs), traceable calibration transducers, and robust, reliable methods for obtaining and analyzing nanoindentation data, so that nanoindentation results can be used with confidence in product design and specification.

\section{Douglas T. Smith}

$\mathrm{N}$ anoindentation is the most commonly used method for determining hardness and elastic properties of small volumes of materials. In this technique, a diamond indenter is pushed into a specimen surface, and the force on and displacement into the surface are recorded. The technique is capable of providing information on the elastic and plastic deformation of a specimen for indentations as shallow as $5 \mathrm{~nm}$ to $10 \mathrm{~nm}$, and requires minimal specimen preparation effort. It is routinely used to measure the mechanical properties of thin films. However, there are only a very limited number of accepted test methods available, and no means to traceably calibrate or verify the performance of nanoindentation instruments.

This leads to large interlaboratory variations in results, particularly for hard, high modulus materials, and prevents the use of nanoindentation in thin film or coating product specifications.

In many mechanical test methods, including nanoindentation, a force is applied to a specimen, and some displacement is measured. Traceable displacement measurement by interferometry is well established. Force measurement is more problematic, however, because the SI unit for force is still based on an artifact kilogram mass. The Microforce Competence Program at NIST has developed a primary realization of force, traceable to electronic and length SI units, for force calibration in the range $1 \mathrm{mN}$ to $10 \mathrm{nN}$. As part of this program, transfer force cells are being developed that will allow force calibration, traceable to NIST, for commercial nanomechanical test equipment such as nanoindentation machines and atomic force microscopes. One such transfer force cell was calibrated against the NIST primary force balance to an uncertainty less than $0.5 \%$ for forces in the range $0.05 \mathrm{mN}$ to $5.0 \mathrm{mN}$. That cell was then mounted, as if it were a specimen, in a popular commercial nanoindentation instrument that had been recently calibrated by its manufacturer using their recommended force calibration procedure. The figure shows the error in the nanoindentation force recorded by the instrument, relative to the traceable force cell reading. For forces above $2 \mathrm{mN}$, the recorded force is within the $1 \%$ uncertainty required by most draft standards for nanoindentation machines. However, for lower applied force, the error increases dramatically, and at $0.05 \mathrm{mN}$, the recorded force is almost $18 \%$ below the actual applied force.

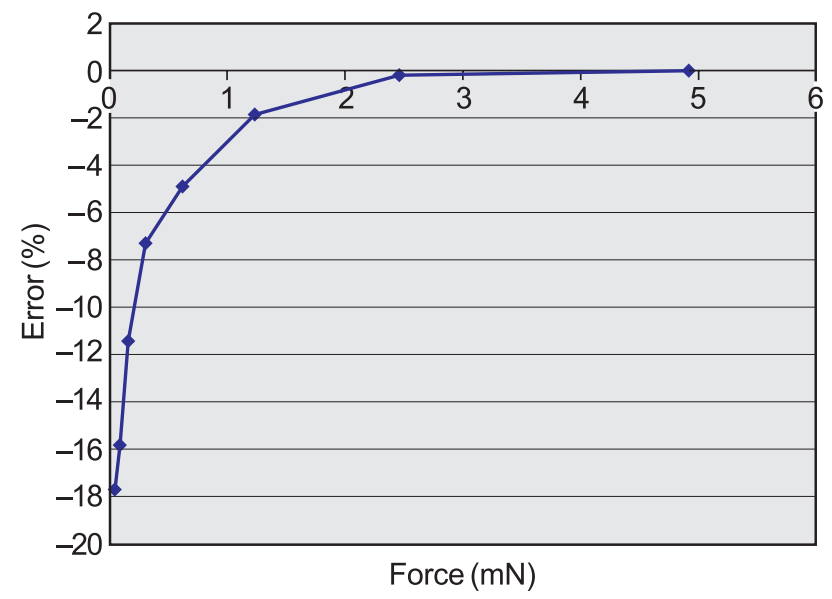

Figure 1: The error in the force applied to a specimen by a commercial nanoindentation instrument, as referenced to a traceably calibrated NIST force cell.

We are also working with both ASTM (E28.06.11) and ISO (TC 164/SC 3) on a wide range of standard test methods for nanoindentation, and an ISO document for the one method has now been approved (ISO 14577). In addition, the Ceramics and Materials Reliability Divisions at NIST are working with the Bundesanstalt für Materialforschung und-prüfung (BAM) in Germany to develop joint thin film SRMs (CRMs, or Certified Reference Materials, in Europe) for use in nanoindentation machine verification.

\section{Contributors and Collaborators}

D. Xiang, B. Hockey, G. Quinn (Ceramics Division, NIST); R. Machado (INMETRO, Brazil); J. Pratt (Manufacturing Engineering Laboratory, NIST); D. Hurley (Materials Reliability Division, NIST); U. Beck (BAM, Germany) 


\section{Nanotribology and Surface Properties}

\begin{abstract}
Accurate adhesion and friction measurements at the nanoscale are emerging as critical issues in device industries and nanotechnology. Measurements characterizing nanometer-thick molecular assemblies and surface textures to control surface properties and to ensure reliability and durability are also needed. Working with device and magnetic storage industries and other agencies, we have designed and built instruments to meet these needs. These instruments are housed in NIST's new Advanced Measurement Laboratory. The facility features vibration isolation, class 1000 clean room standards, and temperature control.
\end{abstract}

\section{Stephen M. Hsu}

$\mathrm{T}$ he nanotribology and surface properties project was initiated as a part of the MSEL Nanotechnology Initiative. The project aims to develop measurement capability for adhesion, friction, and surface forces at the nanoscale. We interact extensively with many industries and research centers in the U.S. and around the world to promote advances in measurement science and seek consensus standard harmonization in three areas: nanocontacts, nanolubrication, and surface texturing.

\section{Nanocontacts}

Adhesion and friction measurements at nanocontacts require accurate detection of normal and lateral forces at nanonewton levels. Scanning probe microscopes use sensitive cantilevers and laser diodes to detect these forces, but this approach introduces rotation of the tips and crosstalk among the xyz planes. To understand the influence of surface forces, many of which are functions of contact area, attention must be focused on determining the real area of contact.

To resolve some of the instrumental challenges, we have engaged instrument makers, such as Hysitron, Veeco, and others, to solicit their inputs and collaborations. At the same time, we have established our own capability in tip fabrication, cantilever spring constant calibration, and tip characterization. Three new instruments were successfully installed as a result of these collaborations: the NIST-Hysitron multiscale friction tester, the NIST nanoadhesion apparatus, and the first prototype of an interferometer microscope from Veeco. We are continuing to work with our partners to develop next-generation instruments as suggested by the Nanometrology Grand Challenges Workshop (NIST, January 2004).
The overall objective of the nanocontacts activity is to develop the constitutive equation of adhesion and friction including the various components of surface forces. With our new adhesion and nanofriction apparatus, we are quantifying the effects of plowing and electrostatic charge on measurements. We continue to work with our external academic partners (UC Berkeley, UC Davis, and Ohio State U) under the Nanotechnology Extramural Initiative to develop friction measurement via three approaches: friction measuring MEMS devices, AFM methods, and ultrahigh vacuum friction measurement. These efforts have been successful, and we have gained considerable understanding of how meniscus forces and electrostatic forces can exert significant effects on nanofriction measurements. A NIST special publication summarizing these findings is under preparation.

\section{Nanolubrication}

Molecular assemblies can be organized to impart hydrophobicity, anti-adhesion, and friction control characteristics on device surfaces. Supported by other agencies and the magnetic storage industry, this activity focuses on how to measure the effects of controlled composition and spacing on properties of nanometerthick films. An ultrahigh vacuum scanning tunneling microscope-atomic force microscope was installed in May 2004 to provide imaging capability at the molecular level. A micro-tribometer was also developed to measure the durability of these films. The synchrotron facility operated by the Division's Characterization Methods Group at Brookhaven National Laboratory continues to be vital in characterizing these complex molecular mixtures.

\section{Surface Texturing}

Surface texture increasingly is being considered as a tool to control surface energy, polarity, adhesion, and friction. Supported by other agencies and industries, we are pioneering the use of specific surface features such as dimples, triangles, and ellipses at microand nanoscale dimensions to supplement molecular assemblies to control surface properties of surfaces. An international cooperative study under the auspices of International Energy Agency (IEA) is underway.

\section{Contributors and Collaborators}

C. Ying, R. Gates, J. Chuang, J. Larson-Basse, L. Ives, D. Fischer (Ceramics Division, NIST); B. Bhushan (Ohio State U.); K. Komoupoulas (UC Berkeley); G. Liu (UC Davis); Y.T. Hsia (Seagate); J. Sengers (U. of Maryland); O. Warren (Hysitron); C. Su (Veeco) 


\section{Materials for Electronics}

The U.S. electronics industry faces strong international competition in the manufacture of smaller, faster, more functional, and more reliable products. Many critical challenges facing the industry require the continual development of advanced materials and processes. The NIST Materials Science and Engineering Laboratory (MSEL) works closely with U.S. industry covering a broad spectrum of sectors including semiconductor manufacturing, device components, packaging, data storage, and assembly, as well as complementary and emerging areas such as optoelectronics and organic electronics. MSEL has a multidivisional approach, committed to addressing the most critical materials measurement and standards issues for electronic materials. Our vision is to be the key resource within the Federal Government for materials metrology development and will be realized through the following objectives:

- Develop and deliver standard measurements and data;

- Develop advanced measurement methods needed by industry to address new problems that arise with the development of new materials;

- Develop and apply in situ as well as real-time, factory floor measurements, for materials and devices having micrometer- to nanometer-scale dimensions;

- Develop combinatorial material methodologies for the rapid optimization of industrially important electronic materials;

- Provide the fundamental understanding of the divergence of thin film and nanoscale material properties from their bulk values;

- Provide the fundamental understanding of materials needed for future nanoelectronic devices, including first principles modeling of such materials.

The NIST/MSEL program consists of projects led by the Metallurgy, Polymers, Materials Reliability, and Ceramics Divisions. These projects are conducted in collaboration with partners from industrial consortia (e.g., International SEMATECH), individual companies, academia, and other government agencies. The program is strongly coupled with other microelectronics programs within the government such as the National Semiconductor Metrology Program (NSMP). Materials metrology needs are also identified through the International Technology Roadmap for Semiconductors (ITRS), the IPC Lead-free Solder Roadmap, the National Electronics Manufacturing Initiative (NEMI) Roadmap, the Optoelectronics Industry Development Association (OIDA) Roadmap, IPC (the International Packaging Consortium), and the National [Magnetic Data] Storage Industry Consortium (NSIC) Roadmap.
In each of these areas, MSEL researchers have made substantial contributions to the most pressing technical challenges facing industry, from new fabrication methods and advanced materials in the semiconductor industry, to advanced packaging materials, to magnetic data storage. Below are just a few examples of MSEL contributions over the past year.

\section{Advanced Gate Dielectrics}

To enable further device scaling, the capacitive equivalent thickness (CET) of the gate stack thickness must be $0.5 \mathrm{~nm}$ to $1.0 \mathrm{~nm}$. This is not achievable with existing $\mathrm{SiO}_{2} /$ polcrystalline $\mathrm{Si}$ gate stacks. Given the large number of possible choices for these new layers, the only feasible approach to understanding the complex materials interactions that result at the gate dielectric/ substrate and gate dielectric/metal gate electrode interfaces is through the application of combinatorial methodologies. This same methodology and apparatus are applicable to a wide variety of problems in the electronic materials field.

\section{Sub-100 nm Nanofabrication}

The continual decrease in feature size has been the driving force for advances in the semiconductor industry. Current structures have $90 \mathrm{~nm}$ dimensions with planned nodes at $65 \mathrm{~nm}$ and $35 \mathrm{~nm}$ structures. Advanced measurements of the patterning materials (photoresists), are needed to enable future large scale manufacturing of smaller devices. MSEL utilizes advanced $\mathrm{x}$-ray and neutron tools to provide insight into the feasibility and optimization of these important processes.

\section{Advanced Metallization}

Electrodeposited copper is rapidly replacing aluminum for on-chip "wiring" because of its lower electrical resistivity, superior electromigration behavior, and the ability to fill fine features without the formation of seams or voids. As feature dimensions go below $100 \mathrm{~nm}$, difficulties in maintaining performance are anticipated. These issues are addressed through a combination of modeling and experimental efforts.

\section{Test Methods for Embedded Passive Devices}

Significant advantages arise if passive devices are integrated directly into the circuit board as embedded passive devices rather than discretely attached with automated assembly. New metrology methods were developed to address the needs of the electronic industry. Two test methods were completed and have received wide acceptance by industry as new methods to accelerate the development of embedded passive device technology.

\section{Contact: Martin L. Green (Ceramics Division),} Eric K. Lin (Polymers Division) 


\section{Combinatorial Tools for Materials Science}

Combinatorial materials science has rapidly become a new paradigm for the acceleration of materials research. It is a fast and efficient methodology for materials optimization and discovery, characterized by high throughput, parallel experiments, automated analysis, and massive data sets. NIST's industrial stakeholders are identifying it as the only viable technique for understanding complex materials science systems in a competitive timeframe. Its application to a critical materials science problem in the Si microelectronics industry, the replacement of the gate stack, is expected to drive an inorganic combinatorial materials science program at NIST.

\section{Martin L. Green}

$\mathrm{N}$ IST/MSEL is poised to play several important roles in developing combinatorial materials methodologies, especially as they pertain to industrially important, advanced inorganic materials. Included in this class are materials such as thermoelectrics, dielectric and metal layers for advanced Si CMOS gate stacks, multiferroics, magnetic semiconductors for spintronics, transparent semiconductors, and fuel cell and $\mathrm{H}_{2}$ storage materials. NIST/MSEL has an opportunity to take a lead position in the use of combinatorial methodologies for:

- New, combinatorially-friendly (rapid, local, microscopic measurements) metrologies;

- Materials optimization and development;

- Means of experimentally verifying computed materials properties;

- Tools for determining phase diagrams and other property data; and

- "Data on demand," or "just-in-time-data."

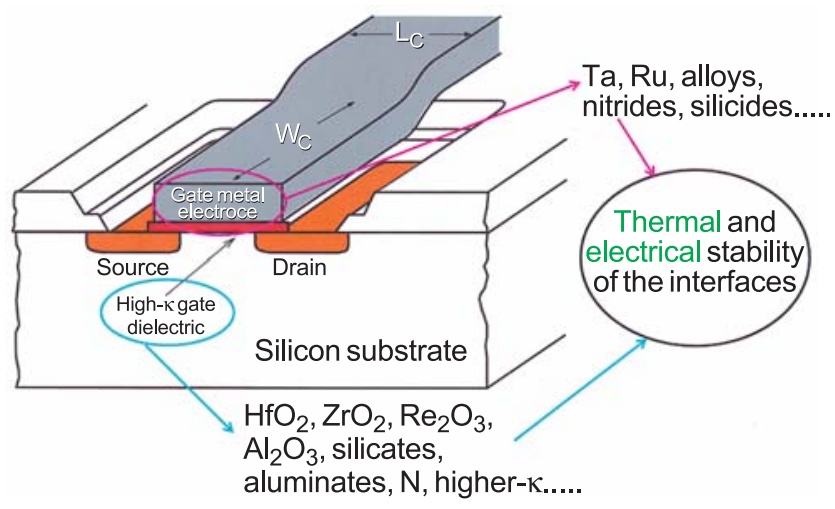

Figure 1: MOSFET device with advanced gate stack.
The advanced gate stack application for Si CMOS is a good example. International Sematech (ISMT) is currently faced with major materials challenges to further miniaturization. Figure 1 schematically illustrates a metal oxide-semiconductor field effect transistor (MOSFET), showing the advanced gate stack which consists of the high- $\kappa(\kappa=$ dielectric constant $)$ gate dielectric and the metal gate electrode. To enable further device scaling, the capacitive equivalent thickness (CET) of the gate stack thickness must be $0.5 \mathrm{~nm}$ to $1.0 \mathrm{~nm}$. This will not be achievable with existing $\mathrm{SiO}_{2}$ / polycrystalline $\mathrm{Si}$ gate stacks. Given the large number of possible choices for these new layers, the only feasible approach to understanding the complex materials interactions that result at the gate dielectric/substrate and gate dielectric/metal gate electrode interfaces is through the application of combinatorial methodologies.

The acquisition of a state-of-the-art combinatorial synthesis tool is expected be one of the cornerstones of the NIST/MSEL effort. In addition, we plan to acquire combinatorially-friendly tools such as a micro-x-ray diffractometer, and to develop new metrologies such as nanocalorimetry for the detection of phase transformations in thin films.

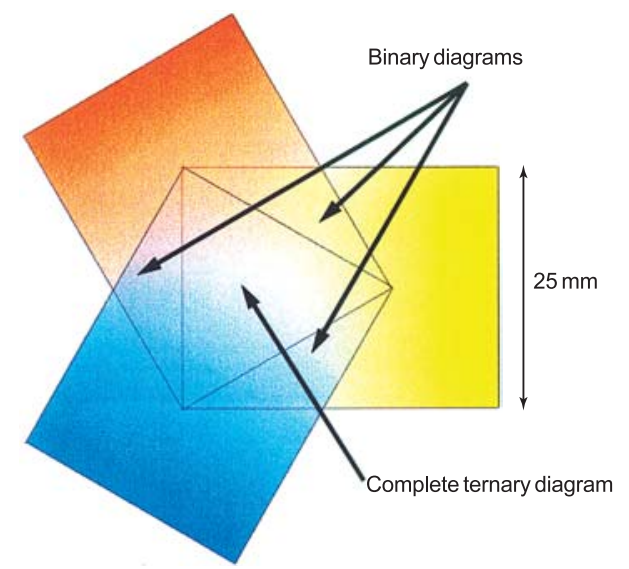

Figure 2: Combinatorial ternary and binary film libraries.

The new combinatorial synthesis tool will be capable of depositing entire ternary and binary libraries in areas as small as $6.5 \mathrm{~cm}^{2}\left(1 \mathrm{in}^{2}\right)$, as is shown in Figure 2.

\section{Contributors and Collaborators}

L. Cook, W. Wong-Ng, P. Schenck (Ceramics Division, NIST); L. Bendersky, A. Davydov (Metallurgy Division, NIST); S. Semancik (CSTL, NIST); J. Suehle, E. Vogel (EEEL, NIST); T. Chikyow (NIMS, Japan); B. Murto (International Sematech); I. Takeuchi (U. of Maryland) 


\section{Spectroscopy, Diffraction, and Imaging of Electronic Materials}

Modern electronic devices have reached the point where fundamental materials properties now limit continued improvement in device performance. Consequently, new materials, their synthesis, their properties, and their interactions with established materials and technologies are in continuous need of development and investigation. The Ceramics Division operates a suite of synchrotron beamlines, at the Advanced Photon Source and at the National Synchrotron Light Source, designed to address critical issues focused on these next-generation materials, including the electronic and structural properties of materials used in advanced electronic applications.

\section{Joseph C. Woicik}

$\mathrm{T}_{\mathrm{p}}^{\mathrm{h}}$ he electronics industry is seeking to increase device performance by increasing carrier mobility in silicon by introducing a small tensile strain into the silicon lattice. This is accomplished by the epitaxial growth of a thin silicon layer on a virtual crystalline substrate of relaxed $\mathrm{Si}_{1-\mathrm{x}} \mathrm{Ge}_{\mathrm{x}}$ with $\mathrm{x}$ typically between $10 \%$ and $50 \%$. A critical aspect of this process is the control of defects in the SiGe-alloy layer and the propagation of these defects into the strained-Si film.

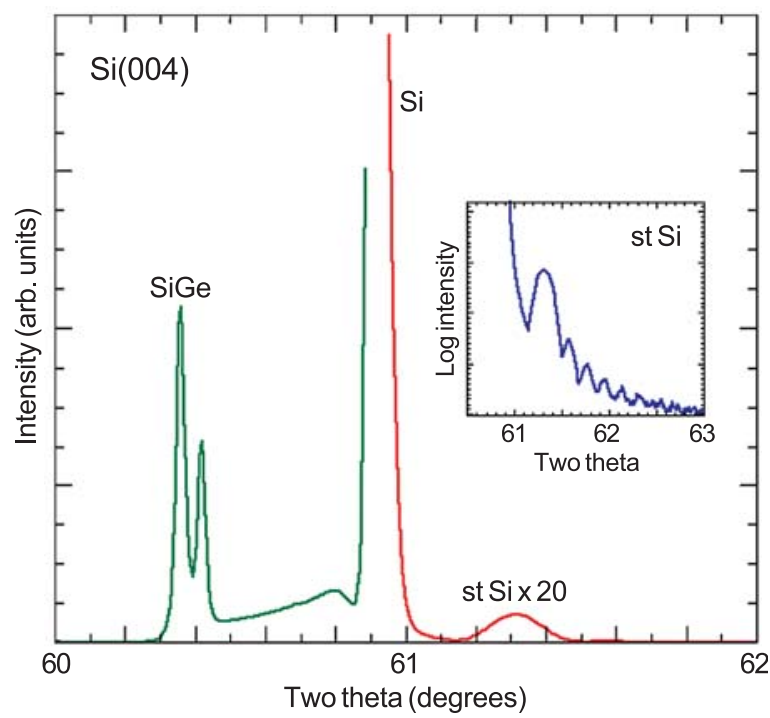

Figure 1: Radial diffraction scan for a strained-Si/Si $i_{x} e_{1-x} / \mathrm{Si}(001)$ semiconductor layered structure.

We have utilized $\mathrm{x}$-ray diffraction and $\mathrm{x}$-ray topography at the UNICAT beamline facility at the Advanced Photon Source to image defects in the SiGe film and to study how these defects propagate into the strained-Si overlayer. Figure 1 shows a high-resolution x-ray diffraction scan around the $\mathrm{Si}(004)$ Bragg condition from a $500 \AA \mathrm{Si}$ film grown on a relaxed $3 \mu \mathrm{m}$ thick SiGe film with a Ge content of $20 \%$. The relaxed SiGe film was grown on top of a SiGe layer that was grown on a $\mathrm{Si}(001)$ substrate and graded in composition from $0 \%$ to $20 \% \mathrm{Ge}$ content over a thickness of $2 \mu \mathrm{m}$.

The diffraction from the SiGe is seen near $2 \theta=60.4^{\circ}$; it appears as a doublet due to a slight composition variation due to the intermediate planarization process. Diffraction from the grade is also apparent. The sharp peak near $2 \theta=60.9^{\circ}$ is diffraction from the Si substrate, and the much weaker peak near $2 \theta=61.3^{\circ}$ is diffraction from the strained-Si film. The Si film has a smaller perpendicular lattice constant than the crystalline Si substrate because it is under in-plane tensile strain due to its epitaxy with the SiGe layer that possesses a larger cubic lattice constant.

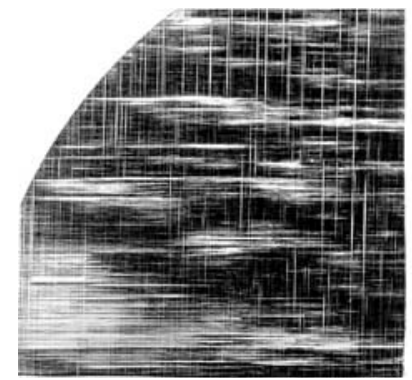

SiGe virtual substrate

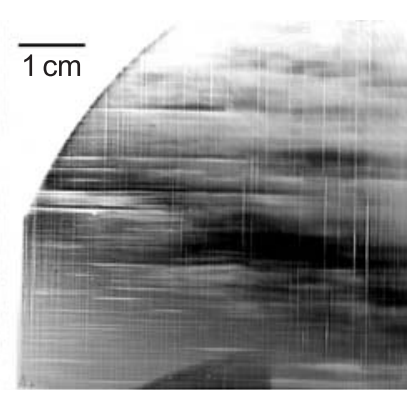

$50 \mathrm{~nm}$ strained silicon film
Figure 2: (113) x-ray topographs of the SiGe film and the strained-Si overlayer.

The differences in lattice constants between the strained-Si film, SiGe layer, and $\mathrm{Si}$ substrate make it possible to image the diffraction from each component separately. Figure 2 shows the (113) x-ray topographs recorded with an x-ray energy of $\approx 8.9 \mathrm{keV}$. This diffraction geometry was chosen because it is within Si total external reflection and, therefore, limits the $\mathrm{x}$-ray penetration depth into the sample.

The microstructure of the substrate and film are nearly identical, demonstrating that the misfit-dislocation structure responsible for the relaxation of the $\mathrm{SiGe}$ layer acts as a template for the microstructure of the strained-Si film. The crystallographic nature of these defects and their contribution to the ultimate relaxation of the strained-Si film are currently under investigation.

\section{Contributors and Collaborators}

D. Black, I. Levin (Ceramics Division, NIST); M. Erdtmann, T.A. Langdo (AmberWave) 


\section{Phase Equilibria and Properties of Dielectric Ceramics}

Ceramic compounds with exploitable dielectric properties are widely used in technical applications such as actuators, transducers, capacitors, and resonators or filters for microwave communications. Phase equilibria determination integrated with systematic chemistry-structure-property studies contribute toward the fundamental understanding and rational design of these technologically important materials with improved properties and/or reduced processing costs.

\section{Terrell A. Vanderah, Igor Levin, and Michael W. Lufaso}

Cost and performance are the primary and secondary drivers, respectively, for today's commercial needs in the area of dielectric ceramics. For example, component suppliers and builders of $2 \mathrm{GHz}$ cellular infrastructure are critically impacted by the high cost of tantalum-containing ceramics needed for dielectric resonators: only a single ceramic material, perovskite-like $\mathrm{Ba}_{3} \mathrm{ZnTa}_{2} \mathrm{O}_{9}(\mathrm{BZT})$, is available with the needed properties. Interest is keen to reduce its processing costs or to find an alternative, less-expensive substitute. The results of an experimental phase equilibria study of the system (Figure 1) revealed that BZT ceramics must be processed so that $\mathrm{ZnO}$ volatilization occurs along the two-phase join between BZT and $\mathrm{Ba}_{8} \mathrm{ZnTa}_{6} \mathrm{O}_{24}(8 \mathrm{~L})$ to prevent the formation of deleterious air-sensitive compounds high in $\mathrm{BaO}$ content, or dielectrically poor "TTB” type phases.

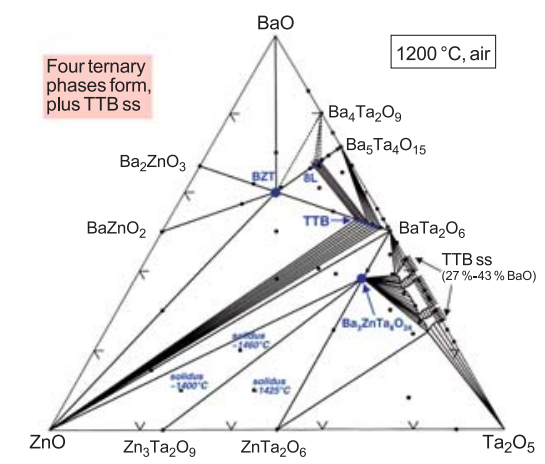

Figure 1: Phase equilibria diagram determined for the system containing the important commercial ceramic $\mathrm{Ba}_{3} \mathrm{ZnTa}_{2} \mathrm{O}_{9}$ (BZT).

A potential replacement for $\mathrm{BZT}$ is the analogous compound with less costly niobium, $\mathrm{Ba}_{3} \mathrm{ZnNb}_{2} \mathrm{O}_{9}$. The phase equilibrium diagram determined for this system (Figure 2) provides processing information for this ceramic and clearly indicates that its dielectric loss cannot be improved by liquid-phase sintering in the presence of low-melting, electrically acceptable $\mathrm{ZnNb}_{2} \mathrm{O}_{6}$, because the two compounds do not occur in equilibrium with each other. Such mixtures, instead, will form dielectrically poor impurity phases.

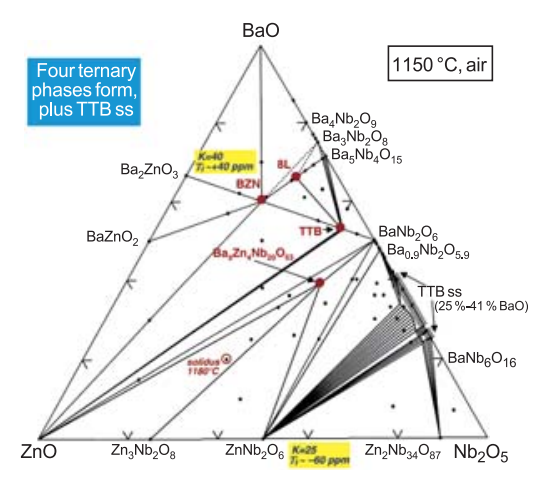

Figure 2: Phase equilibria diagram determined for the system containing $\mathrm{Ba}_{3} \mathrm{ZnNb}{ }_{2} \mathrm{O}_{9}(\mathrm{BZN})$, a possible BZT alternative.

Systematic studies of the $\mathrm{Ba}_{3} \mathrm{MNb}_{2-\mathrm{x}} \mathrm{Sb}_{\mathrm{x}} \mathrm{O}_{9}$ $(\mathrm{M}=\mathrm{Mg}, \mathrm{Ni}, \mathrm{Zn})$, system were carried out to manipulate the crystal chemistry and high-frequency $(>2 \mathrm{GHz})$ dielectric properties by progressive substitution of $\mathrm{Sb}^{5+}$ $\left(d^{10}\right)$ for $\mathrm{Nb}^{5+}\left(d^{0}\right)$. The results showed that control of permittivity and tuning of the temperature coefficient to zero was possible with appropriate substitution. Optimal dielectric losses were obtained for specimens with low $\mathrm{x}$-values and 2:1-type ordered perovskite structures.

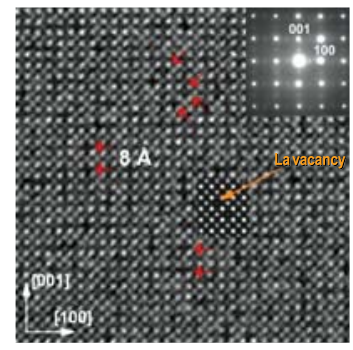

Figure 3: HRTEM image of 50:50 $\mathrm{LaMg}_{1 / 2} \mathrm{Ti}_{1 / 2} \mathrm{O}_{6}-\mathrm{La}_{2 / 3} \mathrm{TiO}_{3}$. Dark crosses denote La vacancies, deduced from image simulations (insert). Short-range order of La vacancies is revealed as pairs of dark crosses in both the [001] and [100] directions and causes diffuse superlattice reflections in the diffraction pattern.

Detailed structural studies of the $\mathrm{LaMg}_{1 / 2} \mathrm{Ti}_{1 / 2} \mathrm{O}_{6^{-}}$ $\mathrm{La}_{2 / 3} \mathrm{TiO}_{3}$ solid solution were carried out to elucidate the origin of the anomalous changes in permittivity and temperature coefficient near 50:50. The results showed that the abrupt changes in properties were accompanied by the disappearance of one octahedral tilting mode and the onset of short-range ordering of La vacancies.

\section{Contributors and Collaborators}

W. Wong-Ng, R.S. Roth, B. Burton, E. Cockayne (Ceramics Division, NIST); J.E. Maslar (Process Measurements Division, NIST); R. Geyer (Radio Frequency Technology Division, NIST); S. Bell (TCI Ceramics) 


\section{Phase Relations of High $\mathrm{T}_{\mathrm{C}}$ Superconductors}

Phase equilibria data are needed for quality control, cost reductions, performance enhancements, and optimal processing in high $T_{c}$ technology. These needs are being addressed through studies of the phase equilibria and kinetics of $\mathrm{Ba}_{2} \mathrm{YCu}_{3} \mathrm{O}_{6+x}$ formation from barium fluoride amorphous precursor films, determination of $\mathrm{Ba}_{2} \mathrm{RCu}_{3} \mathrm{O}_{6+x}$ $(R=D y, Y b)$ phase diagrams, and investigations of superconductor/buffer layer interactions: all play critical roles in the development of RABiTS/IBAD coated conductor technology.

\section{Winnie Wong-Ng, Lawrence P. Cook, and Igor Levin}

$\mathrm{P}$ hase diagrams serve as "blue prints" for successful processing of high $\mathrm{T}_{\mathrm{c}}$ superconductor materials. In the past year, as an integral part of the DOE intensive $R \& D$ program on high $T_{c}$ wire and cable applications, we have continued to provide critical data for the development of practical superconductors. Our research was related to two groups of superconductors: (1) $\mathrm{Ba}_{2} \mathrm{RCu}_{3} \mathrm{O}_{7}$ $(\mathrm{R}=\mathrm{Dy}, \mathrm{Yb})$ coated conductors produced by rolling assisted biaxially textured substrate/ion beam assisted deposition (RABiTS/IBAD); and (2) $\mathrm{MgB}_{2}$.

Our main effort has been focused on the determination of $\mathrm{BaO}-\mathrm{R}_{2} \mathrm{O}_{3}-\mathrm{CuO}_{\mathrm{x}}$ phase relations as a function of oxygen pressure, $\mathrm{p}_{\mathrm{O} 2}$, and choice of lanthanides. These studies were completed under carbonate-free conditions, to match better the processing conditions of RABiTS/IBAD conductors. This year we have completed the study of the two systems with $\mathrm{R}=\mathrm{Dy}$ and $\mathrm{Yb}$. A trend in phase formation and tie-line $\mathrm{Ba}_{2} \mathrm{RCu}_{3} \mathrm{O}_{7}$ relationships was observed in the present work which provides a general correlation for R = Nd, Sm, Eu, Gd, Dy, Ho, Y, Er, and Yb with respect to the size of the lanthanides. In collaboration with Brookhaven National Laboratory and California Institute of Technology, an understanding of the effect of strain on the trend of the orthorhombic to tetragonal phase transition temperature of $\mathrm{Ba}_{2} \mathrm{RCu}_{3} \mathrm{O}_{7}$ was obtained through modeling. By mixing the smaller lanthanides $\mathrm{R}^{\prime}$ with the larger $\mathrm{R}$ in the $\mathrm{Ba}_{2-\mathrm{x}}\left(\mathrm{R}_{1+\mathrm{x}-\mathrm{y}} \mathrm{R}_{\mathrm{y}}^{\prime}\right) \mathrm{Cu}_{3} \mathrm{O}_{6+\mathrm{z}}$ superconductor, both flux-pinning and melting properties can be tailored and optimized. A trend in solid solution extent as related to the size of $\mathrm{R}$ was observed in $\mathrm{Ba}_{2-\mathrm{x}}\left(\mathrm{R}_{1+\mathrm{x}-\mathrm{y}} \mathrm{Y}_{\mathrm{y}}\right) \mathrm{Cu}_{3} \mathrm{O}_{\mathrm{z}}(\mathrm{R}=\mathrm{Sm}, \mathrm{Eu}$ and $\mathrm{Gd})$.

The " $\mathrm{BaF}_{2}$ ex-situ" process is a promising method for production of $\mathrm{Ba}_{2} \mathrm{YCu}_{3} \mathrm{O}_{7}$ superconducting wire and cable. Previously, using a controlled-atmosphere apparatus, we determined the presence of low-temperature liquids in the multicomponent reciprocal $\mathrm{Ba}-\mathrm{Y}-\mathrm{Cu} / / \mathrm{O}, \mathrm{F}$ system. This year, we identified YOF (Figure 1) as an

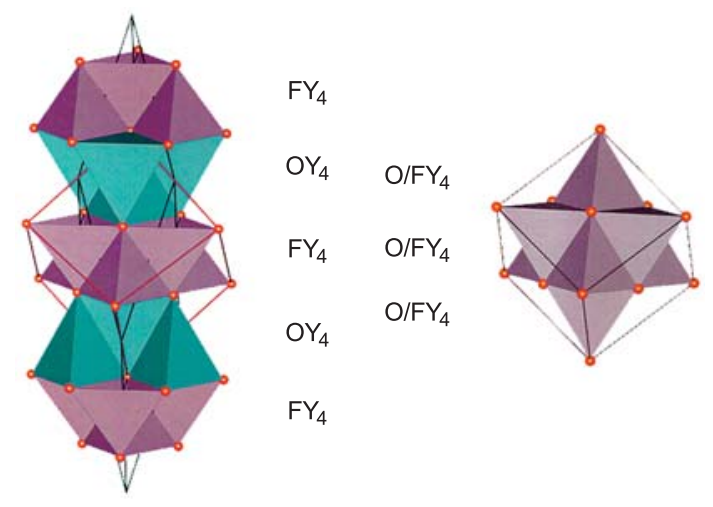

Figure 1: Schematic of the YOF rhombohedral structure (left, showing layers of $\left[\mathrm{OY}_{4}\right]$ and $\left[\mathrm{FY}_{4}\right]$ tetrahedra) and the cubic fluorite structure (right).

important intermediate phase in the system with a low temperature reversible order-disorder phase transition near the initial melting of fluoride-rich melts. These more detailed studies have allowed better definition of the region of low-temperature melting. Also, phase diagrams of the pertinent subsystems of $\mathrm{BaO}-\mathrm{Y}_{2} \mathrm{O}_{3}-\mathrm{CeO}_{2}-\mathrm{CuO}_{\mathrm{x}}$ representing the interaction of product $\mathrm{Ba}_{2} \mathrm{YCu}_{3} \mathrm{O}_{7}$ with the $\mathrm{CeO}_{2}$ buffer layer were determined.

To control film properties, it is important to understand the details of $\mathrm{Ba}_{2} \mathrm{YCu}_{3} \mathrm{O}_{7}$ formation from "BaF 2 " films. High temperature x-ray diffraction and transmission electron microscopy have been applied to compare the detailed conversion mechanism of films prepared with different precursors on $\mathrm{SrTiO}_{3}$ substrates and on RABiTS substrates (both provided by ORNL). A phase with a $\mathrm{Ba}\left(\mathrm{O}_{\mathrm{x}} \mathrm{F}_{\mathrm{y}}\right)$-based superlattice has been found to be involved as an intermediate product. Its presence may prove to be an important factor influencing the kinetics of $\mathrm{Ba}_{2} \mathrm{YCu}_{3} \mathrm{O}_{7}$ formation.

Studies on $\mathrm{MgB}_{2}$ also continued with measurements of the enthalpy of formation (by solution calorimetry) and vapor pressure (by thermogravimetric effusion). Attention was focused on the significant sources of variability in these properties. These data are essential for reproducible processing of $\mathrm{MgB}_{2}$ films, wire and cable.

\section{Contributors and Collaborators}

Q. Huang (NIST Center for Neutron Research); M. Vaudin, P. Schenck (Ceramics Division, NIST); R. Shull (Metallurgy Division, NIST); R. Klein (Biotechnology Division, NIST); R. Feenstra, A. Goyal (ORNL); T. Holesinger (LANL); M. Rupich (ASC); J. Kaduk (BP-Amoco); T. Haugan (U.S. Air Force); P. Canfield (Ames Lab); R. Meng (U. of Houston); D. Welch (BNL), H.B. Su (Caltech) 


\section{Metrology and Standards for Optoelectronic Materials}

The optoelectronics industry needs measurement techniques and accurate materials property data to calibrate their deposition processes and to evaluate both prototype and finished devices. Recent work has focused on the electronic band structure of InGaAsN films (for infrared detectors and solar cells) and calibration of strain in ultrathin $\mathrm{Al}_{x} \mathrm{Ga}{ }_{1-x}$ As films and optical properties of GaN and $\mathrm{ZnO}$ nanowire structures.

\section{Albert J. Paul and Lawrence H. Robins}

$\mathrm{T}_{y} \mathrm{Ga}_{1-y} \mathrm{As}_{1-x} \mathrm{~N}_{x}$ semiconductor alloy films show promise for infrared optoelectronic devices.

Data on the electronic band structure are needed to facilitate device design and simulation. We examined Si-doped $\operatorname{In}_{y} \mathrm{Ga}_{1-y} \mathrm{As}_{1-x} \mathrm{~N}_{x}$ films with $x<0.012$ and $0.052<y<0.075$ by photoreflectance (PR) spectroscopy, supplemented by secondary ion mass spectroscopy (SIMS) and energy dispersive $\mathrm{x}$-ray spectroscopy (EDS, for composition), XRD (strain), and Hall effect (electrical properties). The composition and carrier concentration dependence of the critical point energies, designated $E_{-}$(fundamental gap), $E_{-}+\Delta_{S O}$ (split-off valence band to conduction band), and $E_{+}$(valence band to nitrogen impurity band) was successfully explained by a band anti-crossing and free-carrier band-filling model with a small number of adjustable parameters.
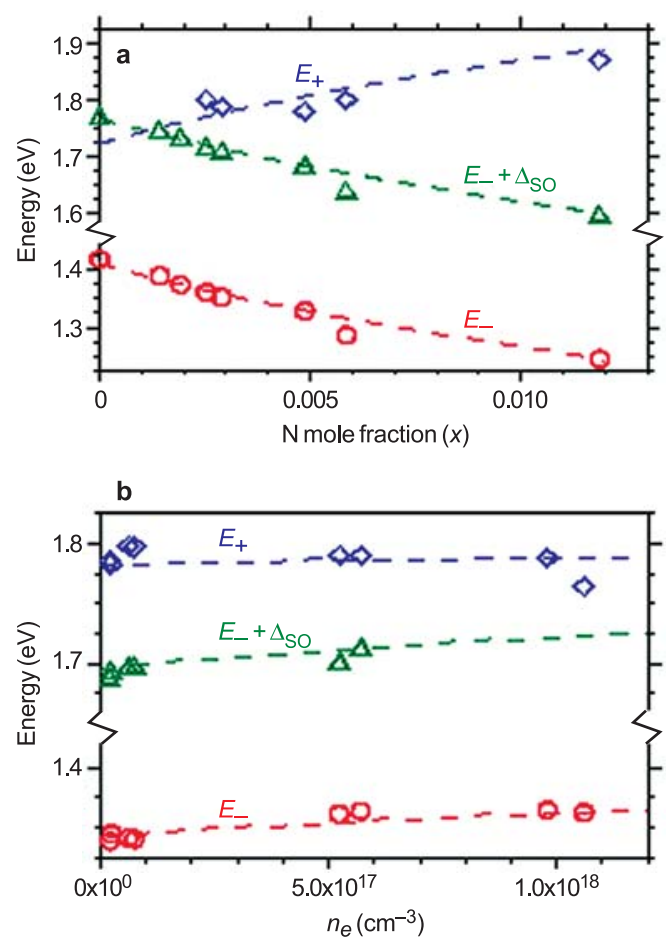

Figure 1: PR critical-point energies: data and modeling.
The measured critical-point energies (symbols) and the best-fit model (dashed curves) are plotted as functions of $\mathrm{N}$ mole fraction $(x)$ in Figure 1(a), and Hall carrier concentration $\left(n_{e}\right)$ in Figure 1(b).

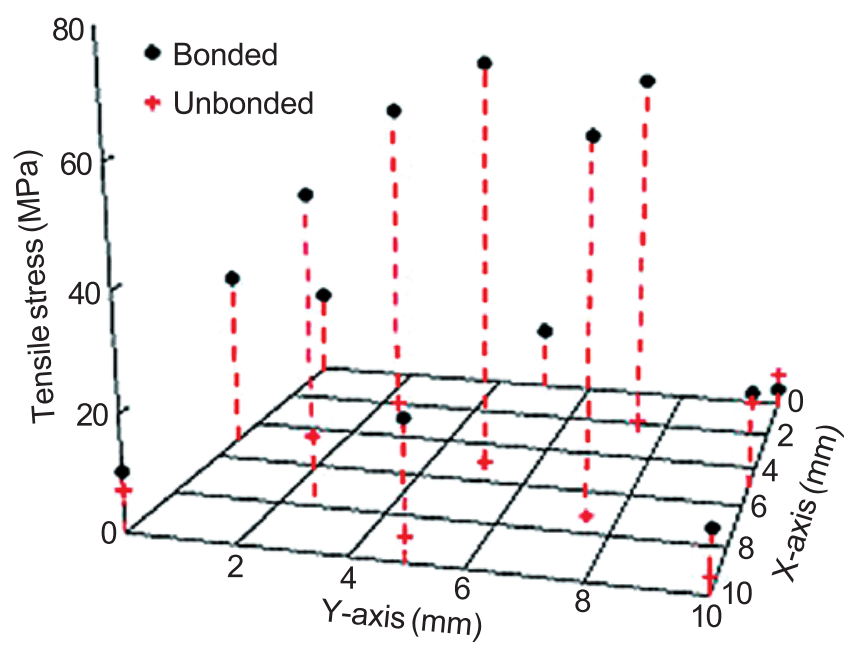

Figure 2: Tensile stress distributions in two $\mathrm{Al}_{0.2} \mathrm{Ga}_{0.8} \mathrm{As}$ structures, obtained from calibrated PL measurements.

We are continuing to develop methods for quantifying optical measurements of stress and strain in III-V semiconductors. By calibrating the shift of the Raman and PL peaks with known applied stress in a load cell, we are able to measure stresses due to differential thermal expansion, film-substrate lattice mismatch, and device packaging. As an example, the stress induced by bonding of a $10 \mathrm{~mm} \times 10 \mathrm{~mm}$ $\mathrm{Al}_{0.2} \mathrm{Ga}_{0.8} \mathrm{As}$-on-GaAs sample (grown as a composition SRM) to a stainless steel disk was measured by this technique. Results from selected locations on the bonded sample and, for comparison, an unbonded piece cut from the same wafer, are shown in Figure 2. A large tensile stress is seen to occur near the center of the bonded piece, but not in the unbonded piece.

Stress measurements of ultrathin ( $5 \mathrm{~nm}$ to $30 \mathrm{~nm}$ ) $\mathrm{AlGaAs}$ films have been initiated to compare spatially resolved measurements of stress near the edges of ultrathin films with the quantitative predictions of Object-Oriented Finite Element (OOF) modeling.

\section{Contributors and Collaborators}

E. Fuller, Y.-S. Kang, G. White (Ceramics Division, NIST); K.A. Bertness, N.A. Sanford (Optoelectronics Division, NIST); A.V. Davydov, A.J. Shapiro (Metallurgy Division, NIST); D. Chandler-Horowitz (Semiconductor Electronics, NIST); M.M.E. Fahmi, S.N. Mohammad (Howard University) 


\section{Experimental and Theoretical Influence of Thin Film Texture on Ferroelectric Hysteresis}

\begin{abstract}
Commercial ferroelectric thin film devices are rapidly approaching the same nanoscale dimensions as individual grains, yet little is known about the influence of grain boundaries and local texture on ferroelectric properties. Accordingly, several complementary experimental and theoretical techniques are being developed to assess the local ferroelectric response. By combining quantitative piezoelectric force microscopy (PFM), electron backscattering diffraction (EBSD), and two-dimensional object oriented finite-element modeling (OOF), various individual crystallographic distributions with either beneficial or detrimental effects can be identified and eventually predicted.
\end{abstract}

\section{Bryan D. Huey, R. Edwin García and John E. Blendell}

Crystallographic orientation can now be determined with nanometer scale lateral resolution using the newly installed electron backscattering diffraction system (EBSD), allowing the orientation of individual ferroelectric grains to be mapped as indicated in Figure 1a.
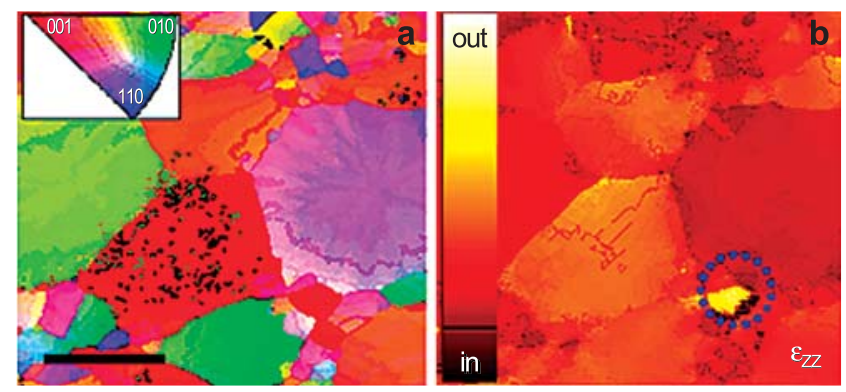

Figure 1: (a) Measured surface orientation of PZT thin film and (b) predicted strain normal to the surface for the same grains when biased with an electrode as indicated. Bar $=20 \mu \mathrm{m}$.

Recent advances in two-dimensional (2-d) object oriented finite element modeling (OOF) have made it possible to predict the piezoelectric response of a polycrystalline thin film with full consideration of the sample texture as determined by EBSD. Figure $1 \mathrm{~b}$ demonstrates the strain normal to the surface for an applied bias of $1 \mathrm{~V}$ across a $100 \mathrm{~nm}$ thick film using a $100 \mathrm{~nm}$ diameter electrode, where the electrode is positioned across multiple grains of differing orientations. The local response is strongly dependent on texture, in some cases oppositely oriented.

Piezoelectric force microscopy (PFM) was employed to determine experimentally the ferroelectric

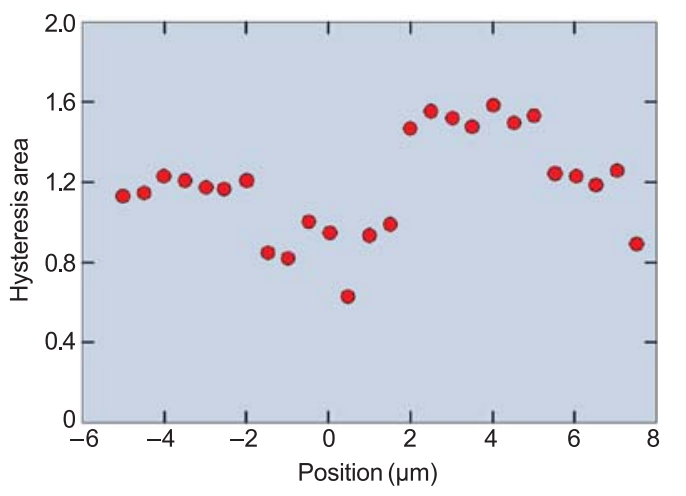

Figure 2: Measured piezoelectric hysteresis areas of several grains/boundaries.

properties of the same grain and grain boundary regions considered by EBSD and OOF. This AFM-based technique is widely used, but can be significantly hindered by several instrumental artifacts that were identified and overcome during this work. The area of piezoelectric hysteresis loops determined using the newly developed quantitative methods is indicated in Figure 2 for several distinct grains and boundaries. The measured hysteresis variations correlate with grains and grain boundaries of differing misorientation as measured by EBSD and correlates with strains calculated by OOF.

Key accomplishments and conclusions include:

1) Novel methods for identifying and overcoming artifacts in piezoelectric force microscopy to allow quantitative local piezoelectric hysteresis measurements;

2) Unique 2-d simulations of converse-piezo actuation with complete incorporation of true thin-film texture;

3) The importance of uniform texture for optimal ferroelectric properties when device dimensions approach the lateral grain size; and

4) The likelihood of domain pinning or even crack initiation at certain grain boundaries and intersections as a result of local orientation.

Results from this work were presented at the SPIE Ferroelectrics conference, American Ceramic Society annual meeting, European Congress on Nanoscale Materials (Nano-04), IEEE Ferroelectrics and Ultrasonics joint meeting, and several invited seminars at NIST and various universities. Publications in the Journal of the American Ceramic Society, Journal of Electroceramics, and Journal of Applied Physics are under review.

\section{Contributors and Collaborators}

M.D. Vaudin, E.R. Fuller, Jr. (Ceramics Division, NIST); S. Hong (Samsung) 


\section{Theory and Modeling of Dielectric Materials}

Dielectric ceramics are widely used in applications such as semiconductor gate dielectrics, actuators, capacitors, and resonators or filters for microwave communications. For simple systems, first-principles (FP) methods allow direct calculations of low-temperature dielectric constants. For complex systems, and for rational new materials design, larger scale modeling is required. Multiscale effective Hamiltonian (EH) models derived from FP calculations retain high accuracy and allow phenomena caused by chemical ordering, defects, and changes in temperature to be explored. The EH provides a bridge from $F P$ calculations $(\leq 100$ atoms) to the needed computations with hundreds of thousands of atoms. We are developing a methodology to enable automatic EH generation for real three-dimensional systems.

\section{Eric Cockayne and Benjamin P. Burton}

$\mathrm{M}$ aterials that are optimized for industrial applications are generally solid solutions in which the configuration of ions on mixed ion sites strongly affects the physical properties. To model these phenomena, detailed FP calculations are performed on a variety of perfectly ordered systems. For example, $\mathrm{PbMg}_{1 / 3} \mathrm{Nb}_{2 / 3} \mathrm{O}_{3}$ (PMN) is the main component of recently discovered materials with ultrahigh piezoelectric constants. PMN presents a singular challenge, because the details of $\mathrm{Mg}-\mathrm{Nb}$ ordering are not fully known. To better understand PMN, we studied various PMN composition supercells with 15 to 30 atoms. A comparison of fully relaxed structures predicts a ground state ordering different from that previously assumed. The two lowest-energy structures found are most compatible with the "random site model" of PMN deduced from experiment. Calculated infrared spectra (directly related to the dielectric function) also show that the two lowest energy structures are most similar to experiment.

For the $\left(\mathrm{CaAl}_{1 / 2} \mathrm{Nb}_{1 / 2} \mathrm{O}_{3}\right)_{1-\mathrm{x}}-\left(\mathrm{CaTiO}_{3}\right)_{\mathrm{x}}(\mathrm{CAN}-\mathrm{CT})$ system of microwave dielectric interest, a cluster expansion EH model for the low temperature dielectric constant was developed to calculate the dielectric constant for an arbitrary CAN-CT configuration as a function of $\mathrm{x}$ and Ti-Al-Nb ordering. Work is in progress to parameterize similar models for other solid solution systems.

Temperature dependent dielectric properties can be modeled with Monte Carlo and molecular dynamics simulations based on EHs. The EH for a heterovalent solid solution includes the random local electric fields generated by mixing differently charged ions in an alloy. We compiled a catalog of the possible local field directions in the nearest-neighbor approximation. By including local fields in an $\mathrm{EH}$ for $\mathrm{PbSc}_{1 / 2} \mathrm{Nb}_{1 / 2} \mathrm{O}_{3}$ (PSN), we can reproduce the experimental observation that the dielectric peak of PSN, as a function of temperature, is broadened when nanoscale ordered domains are present.

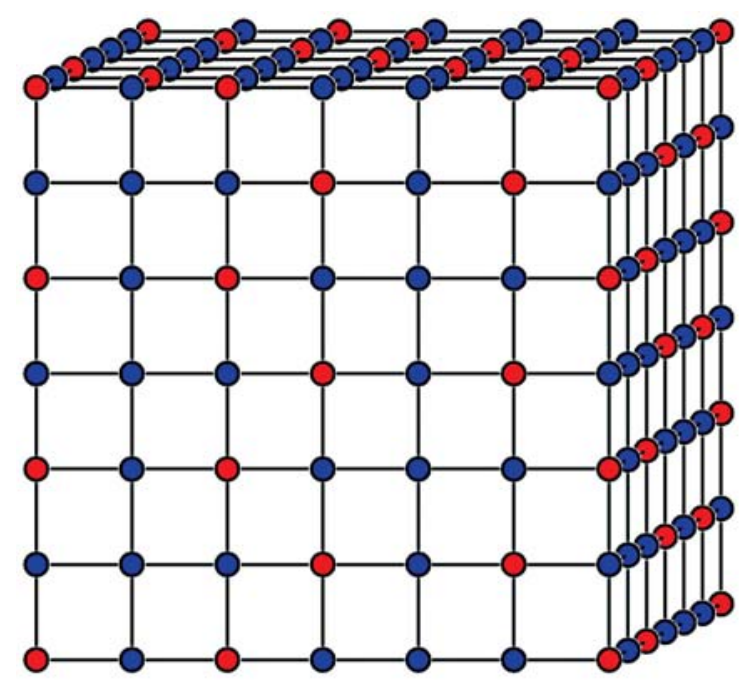

Figure 1: Predicted ground-state ordering of $\mathrm{Mg}$ (red) and $\mathrm{Nb}$ (blue) in $\mathrm{PbMg}_{1 / 3} \mathrm{Nb}_{2 / 3} \mathrm{O}_{3}$.

Defects also affect physical properties. We computed the dipole moment of a $\mathrm{Pb}-\mathrm{O}$ divacancy in $\mathrm{PbTiO}_{3}$ from FP. Its moment is 2.28 e times the distance between the original $\mathrm{Pb}$ and $\mathrm{O}$ sites. The large electric fields produced by such defects have been incorporated into our EH for PSN.

Effective Hamiltonians for dielectrics and ferroelectrics are based on an efficient "lattice Wannier function" (LWF) description of the lattice dynamics of these systems. We have found a principle for automatically generating LWF and have demonstrated that it works on one-dimensional model systems. The method works equally well for chemically ordered systems, disordered systems, and systems with defects. This is a highly promising step towards automatic generation of effective Hamiltonians for real three-dimensional systems, which will make these EH methods accessible to non-experts.

\section{Contributors and Collaborators}

I. Levin, M. Lufaso, S. Tinte (Ceramics Division, NIST); P. Gehrig (NIST Center for Neutron Research, NIST); A. van de Walle (Northwestern U.); S.A. Prosandeev (Rostov State U.); U.V. Waghmare (JNCASR) 


\section{Biomaterials}

New materials and devices are radically changing the treatment of injury and disease, yet it is clear that within this rapidly evolving segment of the materials industry, a basic measurement infrastructure does not exist. The Biomaterials Program develops measurement methods, standards, and fundamental scientific understanding at the interface between the materials and biological sciences. For the health care industry, we focus on dental and medical sectors that apply synthetic materials for replacement, restoration, and regeneration of damaged or diseased tissue. Three primary foci exist within this program: biocompatibility, biomaterials characterization, and materials measurements applied to biological systems.

Whether the medical issue involves implanting a hip- or knee-joint prosthesis, a synthetic bone graft, or a tissue engineering scaffold into the human body, one primary issue is biocompatibility. Using our expertise in materials science, we have developed suitable Reference Materials (RM) for investigating biocompatibility and implant suitability. Research has focused on measuring cellular response to powders and bulk materials that are candidates for implants; recently, we produced a realistic wear particle Standard Reference Material (SRM $\left.{ }^{\circledR} 2880\right)$ for bioactivity testing.

Work on quantitative methods of biomaterials characterization includes assays for adhesion, viability, proliferation, and differentiation of bone cells, 3-dimensional structural/functional imaging of tissue in-growth, and biochemical assays to quantify inflammatory responses to synthetic materials. The focus of this effort is bridging the gap between fundamental knowledge and the product development needs in industry. For example, in collaboration with the Chemical Science and Technology Laboratory, we are developing measurement methodologies and reference materials to assess interactions in complex systems of living cells with synthetic materials. The expected outcome of this work includes reference substrates that induce specific cellular responses, and engineered DNA vectors to act as fluorescent reporters of cellular responses.

Another example of our effort to bridge this gap is our collaboration with the dental industry, which is primarily composed of small manufacturers with limited R\&D capability. Collaborations with the American Dental Association Foundation (ADAF) develop improved materials and materials measurements techniques, patent and license these inventions, and, most importantly, provide a technical foundation. Research focuses on improved understanding of the synergistic interaction of the phases of polymer-based composites and the mechanisms of adhesion to dentin

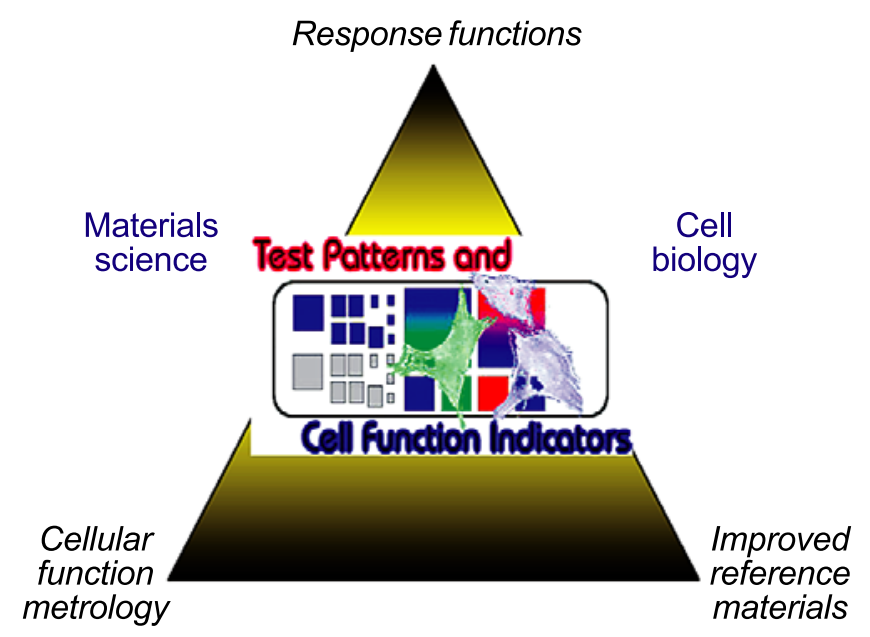

and enamel. This approach will ultimately lead to materials with improved durability, toughness, and adhesion to contiguous tooth structure. We also collaborate with the ADAF to develop metrology for the biocompatibility of synthetic bone grafts.

In this era of interdisciplinary research, we provide an added dimension. By taking a physical/mechanical approach to how cells function, respond, and remodel in interaction with synthetic materials, we provide skill sets typically absent in the biomedical community. Mechanical properties issues also arise when considering synthetic bone grafts and tissue engineering scaffolds. Complementing traditional bulk mechanical property measurements, combinatorial approaches are being developed to identify compositions and surface features that affect properties such as biocompatibility and mechanical durability.

Our mechanical property metrology extends further to biological systems that span the range from individual neurons and muscle cells to complete pulmonary arteries. This necessitates the development of unique mechanical testing platforms and application of a materials science approach to understanding integrated properties. Recently, we have developed a bioreactor capable of applying biaxial stresses and allowing monitoring of the stress and strain of a two-dimensional scaffold sheet during tissue growth.

Fundamental to the Biomaterials program is recognition of the need for an integrated systems approach. Collaborations among and between project teams are critical to progress against the ambitious goals of this program.

Contact: Eric J. Amis (Polymers Division), Stephen M. Hsu (Ceramics Division) 


\section{Controlled Size and Shape UHMWPE Particle SRM for Bioactivity Testing}

The average life of an artificial human joint replacement is about ten to fifteen years. Failures of these joints have been traced to adverse human bioactivity towards wear particles generated inside the body from joint movements. The detailed mechanism of this bioreactivity is well understood, especially the size and shape influence. Conclusions from several industrial workshops held at NIST in the last several years have suggested a strong need for a realistic wear particle reference material for bioactivity testing. We have responded to this need by producing a controlled size and shape UHMWPE wear particle Standard Reference Material, SRM 2880, to be made available in September 2004 for bioactivity testing.

\section{Stephen M. Hsu}

$\mathrm{T}$ o generate wear particles of controlled size and shape, random particle generators such as cryogenic grinding and wear testing cannot be used. Instead, we tested the idea of using surface texturing to provide controlled abrasion. As a preliminary test, we textured a stainless steel surface using abrasives to produce linear and cross-hatched grooves.

Subsequent rubbing of ultrahigh molecular weight polyethylene (UHMWPE) pins against the surface produced promising results, but the particle size and shape distributions were too wide. We then used a semiconductor microfabrication technique to produce specific cutting edges of controlled dimensions using silicon covered with a thin film of chromium for wear resistance. This process, Figure 1, successfully generated suitable particles in distilled water.

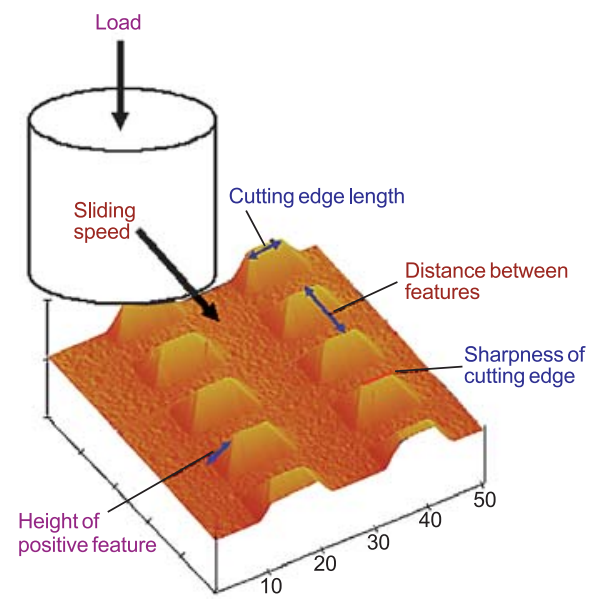

Figure 1: Polyethylene pins rubbing on a textured surface to generate wear particles.
Different surface texture patterns were designed to generate particles of various sizes and shapes. Prof. Paul Wooley of Wayne State University carried out air pouch bioactivity tests in mice and found that elongated particles were more highly reactive than small round particles. The results, published in a biomaterials journal, generated considerable interest in the scientific community.
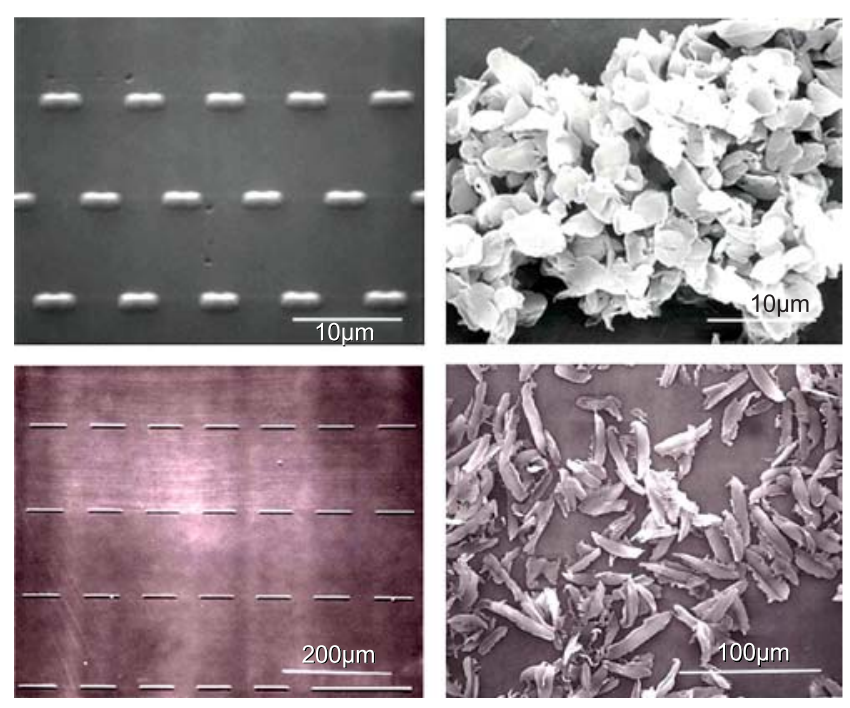

Figure 2: Surface texture features and the resulting particles.

Using this method, different size and shape particles were produced in large quantity under sterile conditions. Two different types of particles were produced: round particles and elongated particles with three different sizes. The particles were packaged in sealed amber ampoules under an inert atmosphere, to avoid oxidation, and were certified in terms of average diameter, aspect ratio, and the minimum number of particles per ampoule. To avoid potential cell culture influence, no dispersant was used in the SRM, but a dispersant that can be used to provide a more accurate particle count per ampoule was recommended.

A unit package of SRM 2880 consists of two sets of ampoules: one containing round particles and the other containing elongated particles. This SRM is scheduled to be available for distribution (https://srmors.nist.gov/) in September 2004.

\section{Contributors and Collaborators}

Y. Liang, P.P. Kavuri, H-W. Fang (Ceramics Division, NIST); J. Tesk (Polymers Division, NIST); D. Schroder (Biomet); C. Merrit (FDA); P. Wooley (Wayne State University); J. Sangers (U of Maryland) 


\section{Data, Reference Materials, and Measurement Methods}

$\mathrm{T}_{\mathrm{p}}^{\mathrm{h}}$

he Ceramics Division has been a vigorous

participant in support of NIST's core mission

as a Measurement Standards Institute. The Division

has made significant contributions to all aspects of standard reference databases, standard reference materials, and standard measurement methods and practices. As may be observed here, the products established by the Division provide a substantial resource spanning a considerable sector of materials metrology.

\section{DATABASES}

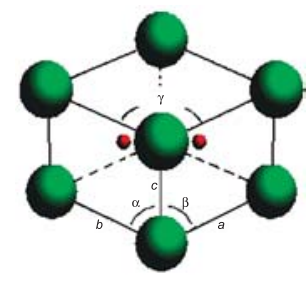

\section{Crystallography}

NIST principal investigator: V.L. Karen

NIST Standard Reference Database 15

NIST/Sandia/ICDD Electron Diffraction Database. Chemical, physical, and crystallographic information for more than 81,500 materials, including minerals, metals, intermetallics, and general inorganic compounds; the database and associated software enable highly selective identification procedures for microscopic and macroscopic crystalline materials. Available for purchase at http://www.nist.gov/srd/nist15.htm.

\section{NIST Standard Reference Database 83}

NIST Structural Database. Crystallographic and atomic position information for metallic crystalline substances, including alloys, intermetallics and minerals; the database is distributed in an ASCII format, convenient for reading into a variety of database management systems or processing by independent software routines. Available for purchase at http://www.nist.gov/srd/nist83.htm.

\section{NIST Standard Reference Database 84}

FIZ/NIST Inorganic Crystal Structure Database, Release 2004/1 (February 2004); produced cooperatively by the Fachinformationszentrum Karlsruhe (FIZ) and NIST; a comprehensive collection of full structural crystallography data of inorganic compounds; contains more than 70,000 entries. Available for purchase at http://www.nist.gov/srd/nist84.htm.

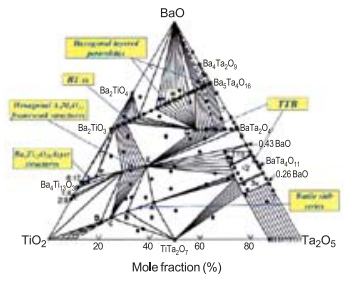

\section{Phase Equilibria Diagrams}

NIST principal investigator: T.A. Vanderah

\section{NIST Standard Reference Database 31}

NIST/ACerS Phase Equilibria Diagrams. Produced jointly by NIST and the American Ceramic Society; thirteen regular book volumes, four topical volumes, three annual volumes, and a computerized database on CD ROM; more than 53,000 units have been sold; the current CD contains approximately 20,000 critically evaluated diagrams and 15,000 expert commentaries. (Free demo CD available from NIST.) Available for purchase at http://www.nist.gov/srd/nist31.htm.

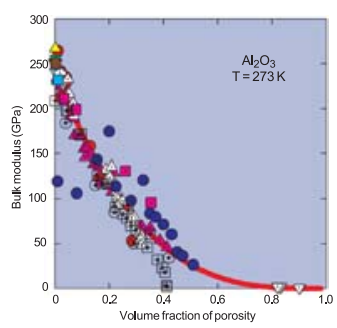

\section{Materials Properties}

NIST principal investigator: R.G. Munro

\section{NIST Standard Reference Database 30}

Structural Ceramics Database. Physical, mechanical, and thermal properties; more than 38,000 numeric values. Available online at http://www.ceramics.nist.gov/srd/scd/scdquery.htm.

\section{NIST Standard Reference Database 62}

High Temperature Superconductors. Physical, mechanical, thermal, and superconducting properties; more than 30,000 numeric values. Available online at http://www.ceramics.nist.gov/srd/hts/htsquery.htm. 


\section{NIST Property Data Summaries}

Focused studies with comprehensive property sets for specific materials and topical studies focused on one property for a wide range of materials.

Available as follows:

- Alumina, http://www.ceramics.nist.gov/srd/ summary/scdaos.htm

- Silicon Carbide, http://www.ceramics.nist.gov/srd/ summary/scdscs.htm

- Titanium Diboride, http://www.ceramics.nist.gov/ srd/summary/scdtib2.htm

- Yttrium Barium Copper Oxide, http:// www.ceramics.nist.gov/srd/summary/htsy123.htm

- Elastic Moduli Data, http://www.ceramics.nist.gov/ srd/summary/emodox00.htm

- Fracture Toughness Data, http:// www.ceramics.nist.gov/srd/summary/ftmain.htm

- Fracture Data for Oxide Glasses, http:// www.ceramics.nist.gov/srd/summary/glsmain.htm

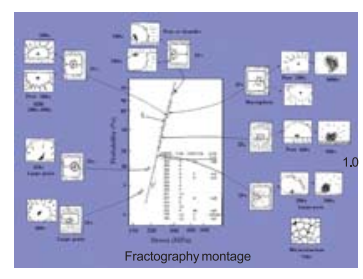

\section{Fractography}

NIST principal investigator: G.D. Quinn

\section{Characterization of Fracture Origins}

Provides an efficient and consistent methodology to locate and characterize fracture origins in advanced ceramics. May be used in conjunction with ASTM standard C-1322. Available online at http://www.ceramics.nist.gov/webbook/fracture/ fracture.htm.

\section{STANDARD REFERENCE MATERIALS}

SRMs produced by the Ceramics Division are available for purchase at http://ts.nist.gov/ts/htdocs/ 230/232/232.htm.

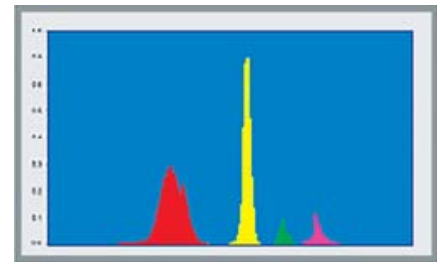

Particle Size Metrology SRMS

NIST principal investigator: J. Kelly

Standard Reference Material 1021

Glass Beads - Particle Size Distribution, a particle size standard for size range $2 \mu \mathrm{m}$ to $12 \mu \mathrm{m}$.

\section{Standard Reference Material 1003c}

Glass Beads - Particle Size Distribution, a particle size standard for size range $20 \mu \mathrm{m}$ to $50 \mu \mathrm{m}$.

\section{Standard Reference Material 1004b}

Glass Beads - Particle Size Distribution, a particle size standard for size range $40 \mu \mathrm{m}$ to $150 \mu \mathrm{m}$.

\section{Standard Reference Material 10176}

Glass Beads - Particle Size Distribution, a particle size standard for size range $100 \mu \mathrm{m}$ to $400 \mu \mathrm{m}$.

\section{Standard Reference Material 1018b}

Glass Beads - Particle Size Distribution, a particle size standard for size range $220 \mu \mathrm{m}$ to $750 \mu \mathrm{m}$.

\section{Standard Reference Material 1019b}

Glass Beads - Particle Size Distribution, a particle size standard for size range $750 \mu \mathrm{m}$ to $2450 \mu \mathrm{m}$.

\section{Standard Reference Material 659}

Particle Size Distribution for Sedigraph Calibration, a particle size standard for size range $0.2 \mu \mathrm{m}$ to $10 \mu \mathrm{m}$.

\section{Standard Reference Material $\mathbf{8 0 1 0}$}

Sand for Sieve Analysis.

\section{Standard Reference Material 1982}

Zirconia Thermal Spray Powder - Particle Size Distribution, a particle size standard for size range $10 \mu \mathrm{m}$ to $150 \mu \mathrm{m}$.

\section{Standard Reference Material 1984}

Thermal Spray Powder - Particle Size Distribution, Tungsten Carbide/Cobalt (Acicular), a particle size standard for size range $9 \mu \mathrm{m}$ to $30 \mu \mathrm{m}$.

\section{Standard Reference Material 1985}

Thermal Spray Powder - Particle Size Distribution, Tungsten Carbide/Cobalt (Spheroidal), a particle size standard for size range $18 \mu \mathrm{m}$ to $55 \mu \mathrm{m}$. 


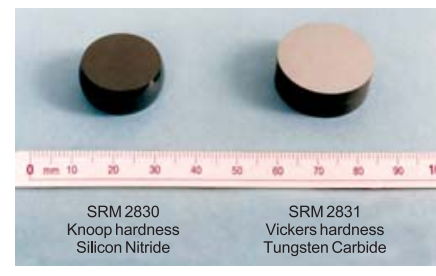

Mechanical Properties SRMs

NIST principal investigator:

G.D. Quinn

Standard Reference Material 2830

Knoop Hardness of Ceramics.

\section{Standard Reference Material 2831}

Vickers Hardness of Ceramics and Hardmetals.

Standard Reference Material 2100

Fracture Toughness of Ceramics.

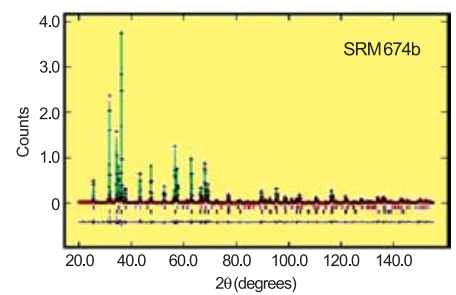

\section{X-Ray Metrology SRMS \\ NIST principal \\ investigator: \\ J. Cline}

\section{Standard Reference Material 640c}

Silicon Powder Line Position/Profile SRM, silicon powder, used for calibration of line position and characterization of the instrument profile function, certified with respect to lattice parameter.

\section{Standard Reference Material 660a}

$\mathrm{LaB}_{6}$ Powder Line Position/Profile SRM, LaB 6 powder, used to characterize the instrument profile function and calibrate line position, certified with respect to lattice parameter.

\section{Standard Reference Material 675}

Mica Powder Line Position (Low Angle) SRM, synthetic fluorophlogopite mica powder, used to characterize the instrument line position at low two-theta angle, certified with respect to lattice parameter.

\section{Standard Reference Material 1976a}

Instrument Response, a sintered alumina plate, certified with respect to lattice parameter and diffraction intensity as a function of two-theta angle (texture), used for general calibration of diffraction equipment, with respect to line position and intensity, via conventional data analysis methods.

\section{Standard Reference Material 676}

Alumina Powder for Quantitative Analysis, high purity alumina powder for general quantitative analyses via powder diffraction methods, certified with respect to lattice parameter.

\section{Standard Reference Material 1878a}

Quantification of Alpha Quartz, respirable $(5 \mu \mathrm{m})$ powders, certified with respect to amorphous content, used primarily by the industrial hygiene community for quantification of quartz in airborne dust.

\section{Standard Reference Material 1879a}

Quantification of Cristobalite, respirable $(5 \mu \mathrm{m})$ powders, certified with respect to amorphous content, used primarily by the industrial hygiene community for quantification of cristobalite in airborne dust.

\section{Standard Reference Material 674b}

Quantitative Analyses, four powders, $\mathrm{Cr}_{2} \mathrm{O}_{3}, \mathrm{CeO}_{2}$, $\mathrm{TiO}_{2}$ and $\mathrm{ZnO}$, allows the user to match the linear attenuation of the standard to that of the unknown, certified for phase purity using neutron time-of-flight diffraction. Supplemental information will include the reference intensity ratio (RIR) or I/Ic value and the lattice parameters as determined with conventional $\mathrm{x}$-ray diffraction.

\section{Standard Reference Material 656}

$\mathrm{Si}_{3} \mathrm{~N}_{4}$ Powder for Quantitative Analysis, two samples of high purity silicon nitride powder, one high in the alpha phase while the other is high in the beta phase, for quantitative analyses via powder diffraction methods, certified with respect to phase purity and the alpha to beta phase ratio.

\section{Standard Reference Material 2910}

Calcium Hydroxyapatite, calcium hydroxyapatite powder for use in evaluating calcium apatites, primarily in the field of biological research, certified with respect to lattice parameters and phase purity.

\section{Standard Reference Material 1979}

Crystallite Size/Line Broadening, $\mathrm{CeO}_{2}$ and $\mathrm{ZnO}$ powder which exhibits diffraction line profile broadening due to crystallite size effects, certified with respect to particle size via XRD line profile analysis, applicable to a range of materials research and industrial interests concerned with crystallite size determination via powder diffraction techniques. 


\section{STANDARD TEST METHODS}

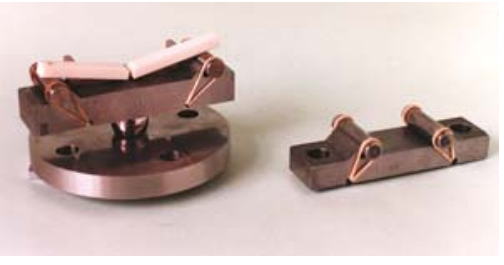

\section{Mechanical \\ Property Test Methods \\ NIST principal investigator: \\ G.D. Quinn}

\section{ASTM C 1161 (2002)}

Standard Test Method for Flexural Strength of Advanced Ceramics at Ambient Temperature.

\section{ASTM C 1322 (2002)}

Standard Practice for Fractography and Characterization of Fracture Origins in Advanced Ceramics.

ASTM C 1326 (2003)

Standard Practice for Knoop Hardness of Advanced Ceramics.

\section{ASTM C 1327 (2003)}

Standard Practice for Vickers Hardness of Advanced Ceramics.

\section{ASTM F 2094 (2001)}

Standard Specification for Silicon Nitride Bearing Balls. This standard addresses the basic quality, physical and mechanical properties, and test requirements for silicon nitride balls used for ball bearings and other specialty applications.
ASTM C 1211 (2002)

Standard Test Method for Flexural Strength of Advanced Ceramics at Ambient Temperature.

ISO Standard 14704 (2000)

Fine Ceramics (Advanced Ceramics, Advanced Technical Ceramics) - Test Method for Flexural Strength of Monolithic Ceramics at Room Temperature.

\section{ISO Standard 18756 (2003)}

Fine Ceramics (Advanced Ceramics, Advanced Technical Ceramics) - Determination of Fracture Toughness of Monolithic Ceramics at Room Temperature by the Surface Crack in Flexure (SCF) Method.

\section{ISO Standard 17565 (2004)}

Fine Ceramics (Advanced Ceramics, Advanced Technical Ceramics) - Test Method for Flexural Strength of Monolithic Ceramics at Elevated Temperature. 


\section{Ceramics Division FY04 Annual Report Publication List}

Abraham, D.P., R.D. Twesten, M. Balasubramanian, J.Kropf, D. Fischer, J.McBreen, I.Petrov, and K.Amine, "Microscopy and Spectroscopy of Lithium Nickel Oxide Based Particles used in High-Power Lithium-Ion Cells," Journal of the Electrochemical Society 150 [11], pp. A1450-A1456 (2003).

Antipov, Y.A., T.-J. Chuang, and H. Gao, "On the Integro-Differential Equation associated with Diffusive Crack Growth Theory," Quarterly Journal of Applied Math. and Mechanics 56 [2], pp. 289-310 (2003).

Banerjee, S., T.Hemraj-Benny, M.Balasubramanian, D.A. Fischer, J.A. Misewich, and S.S. Wong, "Ozonized Single-walled Carbon Nanotubes Investigated using NEXAFS Spectroscopy," Chemical Communications, Issue 7, pp. 772-773 (2004).

Bhushan, B., H. Liu, and S.M. Hsu, "Adhesion and Friction Studies of Silicon and Hydrophobic and Low Friction Films and Investigation of Scale Effects," ASME J. of Tribology 126, pp. 1-8 (2004).

Burnett, D.J., A.M. Gabelnick, D.A. Fischer, S.M. Yalisove, and J.L. Gland, "Defect Enhanced Carbon Monoxide Oxidation at Elevated Oxygen Pressures on a Pt $/ \mathrm{Al}_{2} \mathrm{O}_{3}$ Thin Film," J. Phys. Chem. B 108, pp. 5314-5323 (2004).

Burton, B.P., E. Cockayne, and U. Waghmare, "Random Fields in $\mathrm{A}\left(\mathrm{B}_{1 / 2}{ }^{3+} \mathrm{B}_{1 / 2}{ }^{5+}\right) \mathrm{O}_{3}$ and $\mathrm{A}\left(\mathrm{B}_{1 / 3}{ }^{2+} \mathrm{B}_{2 / 3}{ }^{5+}\right) \mathrm{O}_{3}$ Relaxor Ferroelectrics," TMS Letters 1, pp. 29-30 (2004).

Claessen, R., W. Drube, L. Kover, P. Lefevre, V.N. Strocov, J.C. Woicik, and J. Zegenhagen, "High-Energy Photoelectron Spectroscopy," PETRA III Technical Design Report (TDR), http://www-hasylab.desy.de/facility/upgrade/ petra_tdr.htm, Hasylab, Hamburg, Germany (2004).

Claeys, C.L., W. Wong-Ng, and K.M. Nair, eds., Proceedings of Electrochemical Society/American Ceramic Society Symposium on Low Temperature Electronics and Low-Temperature Cofired Ceramics Based Electronic Devices, October 12-17, 2003, Orlando, FL, Electrochemical Society/American Ceramic Society (2003).

Cockayne, E., "First Principles Calculations of the Dielectric Properties of Perovskite-Type Materials," Journal of the European Ceramic Society 23, pp. 2375-2379 (2003).

Cockayne, E., and B.P. Burton, "Dipole Moment of a Pb-O Vacancy Pair in $\mathrm{PbTiO}_{3}$," Phys. Rev. $B$ 69, Art. No. 144116 (2004).
Devadoss, C., Y. Wang, R. Puligadda, J.L. Lenhart, E.L. Jablonski, D.A. Fischer, S. Sambasivan, E.K. Lin, and W.L. Wu, "Investigation of BARC-resist interfacial interactions," Proceedings of SPIE Optical Microlithography, February 25-28, 2003, Santa Clara, CA, SPIE Vol. 5040, pp. 912-922 (2003).

Dobbins, T.A., A.J. Allen, J. Ilavsky, G.G. Long, P.R. Jemian, A. Kulkarni, and H. Herman, "Recent Developments in the Characterization of Anisotropic Void Populations in Thermal Barrier Coating using Ultra-Small Angle X-ray Scattering," Ceramic Engineering and Science Proc. 24 [3], edited by H.-T. Lin and S. Mrityunjay, American Ceramic Society, pp. 517-524 (2003).

Fahmi, M.M., A. Khan, J.A. Griffin, G.L. Harris, L.H. Robins, A.G. Birdwell, Y.S. Kang, D.T. Smith, T. Steiner, and S. Noor Mohammad, "Nitrogen-Activated Bowing of Dilute $\operatorname{In}_{\mathrm{y}} \mathrm{Ga}_{1-\mathrm{y}} \mathrm{As}_{1-\mathrm{x}} \mathrm{N}_{\mathrm{x}}$ based on Photoreflectance Studies," J. Appl. Phys. 94, pp. 7576-7580 (2003).

Fang, H.-W., S.M. Hsu, and J.V. Sengers, "A Study of the Mechanism of UHMWPE Particle Generation with Microfabricated Surface Features: In situ Observation of Single-Tip Sliding Tests," Proceedings of the 7th World Biomaterials Congress, Sydney, Australia, May 17-21, 2004, Society for Biomaterials, pp. 341 (2004).

Fang, H.-W., S.M. Hsu, and J.V. Sengers, "Analysis of UHMWPE Particle Shape Effects on Bioactivity from the Physical Viewpoint of Phagocytosis Process," Proceedings of the 7th World Biomaterials Congress, Sydney, Australia, May 17-21, 2004, Society for Biomaterials, pp. 1328 (2004).

Fang, H.-W., S.M. Hsu, and J.V. Sengers, "Generation of Narrowly Distributed Ultra-High Molecular Weight Polyethylene Particles by Surface Texturing Techniques," J. of Biomedical Materials Research 67B, pp. 741-749 (2003).

Fang, H.-W., S.M. Hsu, and J.V. Sengers, "Surface-Texture Design to Generate Narrowly Distributed UHMWPE Particles with Specific Sizes and Shapes," Proceedings of the 7th World Biomaterials Congress, Sydney, Australia, May 17-21, 2004, Society for Biomaterials, pp. 1131 (2004).

Fang, H.-W., S.M. Hsu, and J.V. Sengers, "Surface texture design to generate specific sizes and shapes of UHMWPE wear particles," Mat.-wiss. $u$. Werkstofftech 34 [10/11], pp. 976-988 (2003). 
Fang, H.-W., S.M. Hsu, and J.V. Sengers, "Ultra-High Molecular Weight Polyethylene Wear Particle Effects on Bioactivity," NIST-SP 1002 (2003).

Fischer, D.A., K. Efimenko, R.R. Bhat, and S. Sambasivan, "Mapping Surface Chemistry and Molecular Orientation with Combinatorial Near-Edge X-ray Absorption Fine Structure," Macromolecules Rapid Communications 25, pp. 141-149 (2004).

Genzer, J., D.A. Fischer, and K. Efimenko, "Fabricating Two-Dimensional Molecular Gradients via Asymmetric Deformation of Uniformly-Coated Elastomer Sheets," Advanced Materials 15, pp. 1545-1547 (2003).

Hackley, V.A., "Techniques for Measurements in Concentrated Systems: Applications of Electromagnetic Scattering and Ultrasound," Concentrated Colloidal Dispersions: Theory, Experiment and Applications, edited by P. Somasundaran and B. Markovic, American Chemical Society, Washington D.C., Chapter 5, pp. 52-68 (2004).

Hackley, V.A., L. Lum, V. Gintautas, and C.F. Ferraris, Particle Size Analysis by Laser Diffraction Spectrometry: Application to Cementitious Powders, NIST Interagency Report 7097, DoC Technology Administration, National Institute of Standards and Technology, Gaithersburg, MD, (2004).

Haugan, T.J., J.M. Evans, J.C. Tolliver, I. Maartense, P.N. Barnes, W. Wong-Ng, L.P. Cook, and R. Shull, "Flux Pinning and Properties of Solid Solution (Y,Nd) ${ }_{1+\mathrm{x}} \mathrm{Ba}_{2-\mathrm{x}} \mathrm{Cu}_{3} \mathrm{O}_{7-\mathrm{d}}$ Superconductors Processed in Air and Partial Oxygen Atmospheres," Fabrication of Long-Length \& Bulk HTS Conductors, Ceramic Transactions 149, edited by R. Meng, A. Goyal, and W. Wong-Ng, 105th Annual Meeting of the American Ceramic Society (ACerS), April 27-30, 2003, Nashsville, TN, pp. 151 (2004).

Hexemer, A., E. Sivaniah, E.J. Kramer, M.Xiang, X. Li, D.A. Fischer, and C.K. Ober, "Managing Polymer Surface Structure Using Surface Active Block Copolymers in Block Copolymer Mixtures," Journal of Polymer Science, Part-B Polymer Physics 42, pp. 411-420 (2003).

Hsu, S.M., "Molecular Basis of Lubrication," Tribology International 37 [7], pp. 553-559 (2004).

Hsu, S.M., "Nanolubrication: Concept and Design," Tribology International 37 [7], pp. 537-545 (2004).
Ilavsky, J., P.R. Jemian, A.J. Allen, and G.G. Long, "Versatile USAXS (Bonse-Hart) Facility for Advanced Materials Research," Proceedings of Eighth International Conference on Synchrotron Radiation Instrumentation, San Francisco, CA, edited by T. Warwick, et al., American Institute of Physics, pp. 510-513 (2004).

Jablonski, E.L., J.L. Lenhart, S. Sambasivan, D.A. Fischer, R.L. Jones, E.K. Lin, W.L. Wu, D.L. Goldfarb, K. Temple, M.Angelopoulos, and H. Ito, "NEXAFS Measurements of Chemically Amplified Photoresists: Surface versus Bulk Chemistry," Proceedings of 2003 International Conference on Characterization and Metrology for ULSI Technology, March 24-28, 2003, NIST, Gaithersburg, MD, Vol. 683, pp. 439-443 (2003).

Jablonski, E.L., V.M. Prabhu, S. Sambasivan, E.K.Lin, D.A.Fischer, D.L.Goldfarb, M.Angelopoulos, and H. Ito, "NEXAFS Measurements of $157 \mathrm{~nm}$ Photoresist Blends: Surface v. Bulk Chemistry," J. Vac. Sci. Technol. B 21, pp. 3162-3165 (2003).

Jablonski, E.L., S. Sambasivan, E.K. Lin, D.A. Fischer, C. Devadoss, and R. Puligadda, "NEXAFS Measurements of the Interface between Bottom Anti-reflective Coatings and a Model Deprotected Photoresist," J. Vac. Sci. Techno. B 21, pp. 3153-3156 (2003).

Jang, M.-H., W. Wong-Ng, R. Shull, L.P. Cook, and D.S. Suh, "Flux Loss Measurements of Ag-Sheathed Bi-2223 Tapes," American Ceramic Society, Nashville, TN (2003). Fabrication of Long-Length \& Bulk HTS Conductors, Ceramic Transactions 149, edited by R. Meng, A. Goyal, and W. Wong-Ng, 105th Annual Meeting of the American Ceramic Society (ACerS), April 27-30, 2003, Nashsville, TN, pp. 83 (2004).

Kaduk, J.A., W. Wong-Ng, and G.S. Nolas, "X-ray Diffraction Patterns of two Germanium Clathrates, $\mathrm{Sr}_{8} \mathrm{Ga}_{16} \mathrm{Ge}_{30}$ and $\mathrm{Cs}_{8} \mathrm{Na}_{16} \mathrm{Ge}_{136}$ : Promising Candidates for Thermoelectric Applications," The Rigaku Journal 20, pp. 2-11 (2003).

Karen, V.L., and A. Belsky, "FIZ/NIST Inorganic Crystal Structure Database," NIST Standard Reference Database 84, NIST, Gaithersburg, Maryland and FIZ, Karlsruhe, Germany, Release 2003/2 and 2004/1.

Kulkarni, A., A.Goland, H.Herman, A.J.Allen, J. Ilavsky, G.G. Long, C.A. Johnson, and J.A. Ruud, "Microstructure-Property Correlations in Industrial Thermal Barrier Coatings," J. Am. Ceram. Soc 87 [7], pp. 1294-1300 (2004). 
Kulkarni, A., J. Gutleber, S. Sampath, A. Goland, W.B. Lindquist, H. Herman, A.J.Allen, and B. Dowd, "Studies of the Microstructure and Properties of Dense Ceramic Coatings Produced by High-Velocity Oxygen-Fuel Combustion Spraying," Mater. Sci. Eng. A 369, pp. 124-137 (2004).

Larsen-Basse, J., X. Wang, L.K. Ives, and S.M. Hsu, "Some Friction Experiments with Textured Surfaces," Proceedings of the 11th Nordic Symposium on Tribology, Harstadt, Norway, June 1-5, 2004, pp. 751 (2004).

Lee, J., S. Lee, V.A. Hackley, and U. Paik, "Influence of $[\mathrm{Ba}+\mathrm{Ca}] /[\mathrm{Ti}+\mathrm{Zr}]$ Ratio on the Interfacial Property of (Ba,Ca)(Ti, $\mathrm{Zr}) \mathrm{O}_{3}$ (BCTZ) Powders in an Aqueous Medium," Journal of the American Ceramic Society 86 [6], pp. 1034-1036 (2003).

Lee, S., U. Paik, V.A. Hackley, Y-G. Jung, and K-J. Yoon, "Microstructure and Permittivity of Sintered $\mathrm{BaTiO}_{3}$ : Influence of Particle Surface Chemistry in an Aqueous Medium," Materials Research Bulletin 39, pp. 93-102 (2004).

Lenhart, J.L., D.A.Fischer, S. Sambasivan, E.K.Lin, C.L. Soles, R.L. Jones, W.L. Wu, D.L. Goldfarb, and M.Angelopoulos, "Utilizing Near Edge X-ray Absorption Fine Structure to Probe Interfacial Issues in Photolithography," Polymers for Microelectronics and Nanoelectronics, ACS Symposium Series 874, edited by Qinghuang Lin, pp. 98-117 (2004).

Lenhart, J.L., D.A.Fischer, S. Sambasivan, E.K.Lin, R.L. Jones, C.L. Soles, W.L. Wu, D.L. Goldfarb, and M.Angelopoulos, "X-ray Absorption Spectroscopy for Probing Interfacial Issues in Photo-lithography," Proceedings of SPIE Advances in Resist Technology and Processing XX, February 25-28, 2003, Santa Clara, CA, SPIE Vol. 5039, pp. 343 (2003).

Levin, I., T.G. Amos, L. Farber, S.M. Bell, R.S. Roth, T.A. Vanderah, and B.H. Toby, "Phase Equilibria, Crystal Structures, and Dielectric Anomaly in the $\mathrm{BaZrO}_{3}-\mathrm{CaZrO}_{3}$ System," J. Solid State Chem. 175, pp. 170-181 (2003).

Levin, I., R.D. Leapman, M. Kovler, and Y. Roizin, "Beam-Induced Nitrogen Segregation During EELS Measurements on the Silicon Oxide-Nitride-Oxide Stacks," Appl. Phys. Lett. 83 [8], pp. 1548-1550 (2003).

Lufaso, M.W., "Crystal Structure, Modeling, and Delectric Property Relationships of 2:1 Ordered Ba3MM'2O9 ( $\left.\mathrm{M}=\mathrm{Mg}, \mathrm{Ni}, \mathrm{Zn} ; \mathrm{M}^{\prime}=\mathrm{Nb}, \mathrm{Ta}\right)$

Perovskites," Chemistry of Materials 16, pp. 2148-2156 (2004).
Marsh, A.L., D.J. Burnett, D.A. Fischer, and J.L. Gland, "Benzene Intermediates and Mechanisms during Catalytic Oxidation on the Pt (111) Surface Using Soft X-ray Fluorescence Methods," J. Phys. Chem. B 107, pp. 12472-12479 (2003).

Marsh, A.L., D.J. Burnett, D.A. Fischer, and J.L. Gland, "Soft X-ray Studies of Toluene Catalytic Oxidation on the Pt(111) Surface," Journal of Physical Chemistry B 108, pp. 605-611 (2004).

Meng, R., A. Goyal, and W. Wong-Ng, eds., Fabrication of Long-Length \& Bulk HTS Conductors, Ceramic Transactions 149, 105th Annual Meeting of the American Ceramic Society (ACerS), April 27-30, 2003, Nashsville, TN (2004).

Mighell,A.D., "The Normalized Reduced Form and Cell: Mathematical Tools for Lattice Analysis Symmetry and Similarity," J. Res. Natl. Inst. Stand. Technol. 108, pp. 447-452 (2003).

Mighell, A.D., W. Wong-Ng, and G.J. Piermarini, "Howard McMurdie: Going Strong at 98, 60 Years with JCPDS/ICDD and 75 years at NBS/NIST," Powd. Diffr. 18 [3], pp. 271-272 (2003).

Munro, R.G., Ceramics WebBook, Evaluated Data, http://www.ceramics.nist.gov/webbook/ evaluate.htm, Gaithersburg, Maryland (2004).

Paik, U., S. Lee, and V.A. Hackley, "Influence of Barium Dissolution on the Electrokinetic Properties of Colloidal $\mathrm{BaTiO}_{3}$ in an Aqueous Medium," Journal of the American Ceramic Society 86 [10], pp. 1662-1668 (2003).

Prosandeev, S.A., E. Cockayne, and B.P. Burton, "The Energetics of Li Ion Displacements in $\mathrm{K}_{1-\mathrm{x}} \mathrm{Li}_{\mathrm{x}} \mathrm{TaO}_{3}$ : First Principles Calculations," Physical Review B 68, Art. No. 014120 (2003).

Prosandeev, S.A., E. Cockayne, and B.P. Burton, "First-Principles Calculations of Ionic Vibrational Frequencies in $\mathrm{PbMg}_{1 / 3} \mathrm{Nb}_{2 / 3} \mathrm{O}_{3}$," Fundamental Physics of Ferroelectrics 2003, AIP Conference Proceedings Series 637, edited by Peter K. Davies and David J. Singh, pp. 46-151 (2003).

Quinn, G., "Reference Materials Available for Ceramics and Hard Metals," Journal of Thermal Spray Technology 13 [2], pp. 171-171 (2004).

Quinn, G.D., J. Eichler, U. Eisele, and J. Rodel, "Fracture Mirrors in Nanoscale 3Y-TZP," Journal of the American Ceramics Society 87 [3], pp. 513-516 (2004). 
Ren, W., S. Yang, H. Fang, S.M. Hsu, and P.H. Wooley, "Distinct Gene Expression of Nuclear Activator of Nuclear Factor-kB and Rank Ligand in the Inflammatory Response to Variant Morphologies of UHMWPE Particles," J. of Biomaterials 24 [26], pp. 4819-4826 (2003).

Ridley,A.K., M.L. Machesky, V.A.Hackley, M.P. Finnegan, and J.F. Banfield, "Surface Charge Phenomena of Nanocrystalline Anatase," Geochimica et Cosmochimica Acta 67 [18], pp. A398-A398 (2003).

Roth, R.S., and T.A. Vanderah, "Experimental Deterimination of Phase Equilibria Diagrams in Ceramic Systems," Proceedings of the 9th Asian Conference on Solid State Ionics, June 6-11, 2004, Jeju Island, South Korea, World Scientific, Singapore, pp. 3-18 (2004).

Sahiner, M.A., P. Ansari, M.S. Carroll, C.W. Magee, S.W. Novak, and J.C. Woicik, "XAFS as a Direct Local Structural Probe in Revealing the Effects of C Presence in B Diffusion in SiGe Layers," Proceedings of the Materials Research Society, April 12-16, 2004, San Francisco, CA, MRS Proceedings 810, C11.10.1 (2004).

Sahiner, M.A., C.W. Magee, D.F. Downey, E.Arevalo, and J.C. Woicik, "The Role of Ge in Cluster Formation in $\mathrm{B}$ and $\mathrm{BF}_{2}$ Implanted Si Wafers after Ge Pre-amorphization," Proceedings of the Materials Research Society, December 1-5, 2003, Boston, MA, MRS Proceedings 792, R7.3.1 (2004).

Sambasivan, S., D.A. Fischer, M.C. Shen, and S.M.Hsu, "Molecular Orientation of Ultra-high Molecular Weight Polyethylene Induced by Various Sliding Motions," Journal of Biomedical Materials Research Part B: Applied Biomaterials 70 [2], pp. 278-285 (2004).

Sanford, N.A., L.H. Robins, A.V. Davydov, A. Shapiro, D.V. Tsvetkov, A.V. Dmitriev, S. Keller, U.K. Mishra, and S.P. DenBaars, "Refractive Index Study of AlxGa1-xN Films Grown on Sapphire Substrates," J. Appl. Phys. 94, pp. 2980-2991 (2003).

Schenk, P.K., D.L. Kaiser, and A.V. Davydov, "High Throughput Characterization of the Optical Properties of Compositionally Graded Combinatorial Films," Applied Surface Science 223, pp. 200-205 (2004).

Siegrist, T., and T.A.Vanderah, "Combining Magnets and Dielectrics: Crystal Chemistry in the $\mathrm{BaO}-\mathrm{Fe}_{2} \mathrm{O}_{3}-\mathrm{TiO}_{2}$ System," European J. Inorg. Chem. 8, pp. 1483-1501 (2003).

Swider, J.R., V.A. Hackley, and J. Winter, "Characterization of Chinese Ink in Size and Surface," Journal of Cultural Heritage 4, pp. 175-186 (2003).
Tang, Z., A.F. Bower, and T.-J. Chuang, "Numerical Simulations of the Growth and Deflection of a Stress-Corrosion Notch on the Interface between Two Reactive Solids," International Journal of Fracture 127 [1], pp. 1-20, (2004).

Tewari, Y.B., P.D. Gery, M.D. Vaudin, A.D. Mighell, R. Klein, and R.N. Goldberg, "Saturation Molalities and Standard Molar Enthalpies of Solution of Cytidine(cr), Hypoxanthine(cr), Thymidine(cr), Thymine(cr), Uridine(cr), and Xanthine(cr) in $\mathrm{H} 2 \mathrm{O}(1)$, , J. Chem. Thermodynamics 36, pp. 645-658 (2004).

Vanderah, D.J., T. Parr, V. Silin, C.W. Meuse, R.S. Gates, H. La, and G. Valincius, "Isostructural Self-Assembled Monolayers, 2, Methyl 1-(3-Mercaptopropyl)Oligo(Ethylene Oxide)s," Langmuir 20, pp. 1311-1316 (2004).

Vanderah, T.A., V.L. Miller, I.Levin, S.M.Bell, and T. Negas, "Phase Relations, Crystal Chemistry, and Dielectric Properties in Sections of the $\mathrm{La}_{2} \mathrm{O}_{3}-\mathrm{CaO}-\mathrm{MgO}-\mathrm{TiO}_{2}$ System," J. Solid State Chem. 177, pp. 2023-2038 (2004).

Vanderah, T.A., T. Siegrist, R.S. Roth, A.P. Ramirez, and R.G. Geyer, "Crystal Structure and Properties of $\mathrm{Ba}_{11} \mathrm{FeTi}_{27} \mathrm{O}_{66.5}$," Eur. J. Inorg. Chem. 2004, pp. 2434-2441 (2004).

Waghmare, U.V., E. Cockayne, and B.P. Burton, "Ferroelectric Phase Transitions in Nano-Scale Chemically Ordered $\mathrm{PbSc}_{0.5} \mathrm{Nb}_{0.5} \mathrm{O}_{3}$ using a First-Principles Model Hamiltonian," Ferroelectrics 291, pp.187-196 (2003).

Wittberg, T.N., P.S. Wang, and S.M. Hsu, "Surface Oxidation Kinetics of SiC Powders in Wet and Dry Air Studied by X-Ray Photoelectron Spectroscopy and Bremsstrahlung-Excited Auger Electron spectroscopy," Surface and Interface Analysis 35, pp. 773-778 (2003).

Woicik, J.C., "Site-Specific Valence X-ray Photoelectron Spectroscopy," Synch. Rad. News 17, pp. 48-53 (2004).

Woicik, J.C., "Site-Specific X-ray Photoelectron Spectroscopy: A New Method to Measure Partial Density of Valence States," Fundamental Physics of Ferroelectrics 2003, February 2-5, 2003, Williamsburg, VA, edited by P.K. Davies and D.J. Singh, AIP Conference Proceedings 677, 261 (2003).

Wong-Ng, W., L.P. Cook, J. Suh, R. Coutts, J.K. Stalick, I. Levin, and Q. Huang, " $\mathrm{BaO}-\mathrm{Nd}_{2} \mathrm{O}_{3}-\mathrm{CuO}_{x}$ Subsolidus Equilibria Under Carbonate-Free Conditions at $\mathrm{p}_{\mathrm{O} 2}=100 \mathrm{~Pa}$, and at $\mathrm{p}_{\mathrm{O} 2}=21 \mathrm{kPa}$," J. Solid State Chem. 173, pp. 476-488 (2003). 
Wong-Ng, W., L. Swartzendruber, J.A. Kaduk, and L.H. Bennett, "Magnetic and Structural Properties of the 'Brown Phase' Solid Solution $\mathrm{Ba}\left(\mathrm{Nd}_{2-\mathrm{x}} \mathrm{La}_{\mathrm{x}}\right) \mathrm{CuO}_{5}$," Physica C 390, pp. 213-220 (2003).

Wong-Ng, W., L.P. Cook, J. Suh, R. Coutts, J. Stalick, I. Levin, and Q. Huang, " $\mathrm{BaO}-\mathrm{Nd}_{2} \mathrm{O}_{3}-\mathrm{CuO}$ Subsolidus Equilibria Under Carbonate-Free Conditions at $\mathrm{pO}_{2}=100 \mathrm{~Pa}$ and at $\mathrm{pO}_{2}=21 \mathrm{kPa}, " J$. Solid State Chem. 173, pp. 476-488 (2003).

Wong-Ng, W., L.P. Cook, and J. Suh, "Phase Relation in the $\mathrm{BaO}-\mathrm{R}_{2} \mathrm{O}_{3}-\mathrm{CuO}_{\mathrm{x}}$ Systems," Fabrication of Long-Length \& Bulk HTS Conductors, Ceramic Transactions 149, 105th Annual Meeting of the American Ceramic Society (ACerS), April 27-30, 2003, Nashsville, TN, pp. 163-175 (2003).

Wong-Ng, W., L.P. Cook, J. Suh, and J.A. Kudak, "Phase relationships in the $\mathrm{BaO}-\mathrm{Sm}_{2} \mathrm{O}_{3}-\mathrm{CuO}_{\mathrm{x}}$ system under $100 \mathrm{~Pa} \mathrm{O}_{2}$," Physica C 405, pp. 47-58 (2004).

Wong-Ng, W., L.P. Cook, and J. Suh, "Phase Relations in the Ba-R-Cu-O (R = Nd, Sm, Eu, Gd, Ho, Y, and Er) Systems Prepared Under AtmosphericallyControlled Conditions," Proceedings of Frontiers in Superconducting Materials-New Materials and Applications, December 1-5, 2003, Boston, MA, Materials Research Society (MRS), EE8.27 (2003).

Wong-Ng, W., L.P. Cook, I. Levin, M. Vaudin, J. Suh, and R. Feenstra, "Coated Conductors: Phase Relations in the Ba-Y-Cu-F-O-H System," Proceedings of Frontiers in Superconducting Materials-New Materials and Applications, December 1-5, 2003, Boston, MA, Materials Research Society (MRS), EE2.9 (2003).

Wong-Ng, W., "The 2003 Material Research Society (MRS) Fall Meeting Report," Powd. Diffr. 19 [2], pp. 206-208 (2004).
Wong-Ng, W., "Seventh International Conference on Materials and Mechanisms of Superconductivity and High Temperature Superconductors ( $\left.\mathrm{M}^{2} \mathrm{~S}-\mathrm{Rio}\right)$, Powd. Diffr. 19 [2], pp. 209-210 (2004).

Wu, W., S. Sambasivan, C. Wang, W.E. Wallace, J. Genzer, and D.A. Fischer, "A Direct Comparison of Surface and Bulk Chain-Relaxation in Polystyrene," Euro Physics J. E 12, pp. 127-132 (2003).

Yoon, W.S., M. Balasubramanian, X.Q. Yang, Z. Fu, D.A. Fischer, and J. McBreen, "Soft X-ray Absorption Spectroscopic Study of a $\mathrm{LiNi}_{0.5} \mathrm{Mn}_{0.5} \mathrm{O}_{2}$ Cathode during Charge," Journal of the Electrochemical Society 151 [2], pp. A246-A251 (2004).

Yoon, W.S., M. Balasubramanian, X.Q. Yang, D.A. Fischer, and J. McBreen, "A Combined NMR and XAS Study on the Local Environments and Electronic Structures of the Electrochemically Li-ion Deintercalated Li1-xCo1/3Ni1/3Mn1/3O2 Electrode System," Electrochemcial and Solid-State Letters 7 [3], pp. A53-A55 (2004).

You, J., X. Wang, P. Schenck, T. Vanderah, M. Harne, C. Cedeno, N. Swanson, and E. Farabaugh, "Phase Equilibria Diagrams CD-ROM Database," NIST Standard Reference Database 31, The American Ceramic Society, Westerville, OH, Version 3.0 (March 2004).

Zhang, K., G. Rosenbaum, R. Liu, C. Liu, and D.A. Fischer, "Development of Multilayer Analyzer Array Detectors for X-ray Fluorescence at the Third Generation Synchrotron Source," Proceedings Synchrotron Radiation Instrumentation: Eighth International, edited by T. Wawick, et al., August 25-29, 2003, San Francisco, CA., pp. 957-960 (2004). 



\section{Ceramics Division}

\section{Chief}

Debra L. Kaiser

Phone: 301-975-6119

E-mail: debra.kaiser@nist.gov

\section{Deputy Chief}

\section{Ronald G. Munro}

Phone: 301-975-6127

E-mail: ronald.munro@nist.gov

Group Leaders

\section{Characterization Methods}

Daniel A. Fischer

Phone: 631-344-5177

E-mail: daniel.fischer@nist.gov

\section{Data and Standards Technology}

Ronald G. Munro

Phone: 301-975-6127

E-mail: ronald.munro@nist.gov

Electronic and Optoelectronic Materials

\section{Martin L. Green}

Phone: 301-975-8496

E-mail: martin.green@nist.gov

\section{Nanomechanical Properties}

Douglas T. Smith

Phone: 301-975-6020

E-mail: douglas.smith@nist.gov

\section{Nanotribology}

Stephen M. Hsu

Phone: 301-975-6120

E-mail: stephen.hsu@nist.gov 


\section{Research Staff}

Allen, Andrew

andrew.allen@nist.gov

Small-angle $x$-ray scattering

Small-angle neutron scattering

Ceramic microstructural analysis

Black, David

david.black@nist.gov

Defect microstructures

$\mathrm{X}$-ray imaging

Blendell, John

john.blendell@nist.gov

Ceramic processing

Sintering and diffusion-controlled processes

Burdette, Harold

harold.burdette@nist.gov

$\mathrm{X}$-ray optics

X-ray diffraction imaging

Instrumentation

Burton, Benjamin

benjamin.burton@nist.gov

Calculated phase diagrams

Dielectric ceramics

Chuang, Tze-Jer

tze-jer.chuang@nist.gov

Finite-element modeling

Lifetime predictions

Nanocontact mechanics

Cline, James

james.cline@nist.gov

Standard reference materials

High-temperature $\mathrm{x}$-ray diffraction

Microstructural effects in $\mathrm{x}$-ray diffraction

Rietveld refinement of $x$-ray diffraction data

Cockayne, Eric

eric.cockayne@nist.gov

First-principles methods

Phase equilibria and properties of dielectrics

Cook, Lawrence

lawrence.cook@nist.gov

High-temperature chemistry

Phase equilibria

DeSanto, Peter

peter.desanto@nist.gov

Electronic ceramics

Solid oxide catalysts

Crystal structure/property relationships
Fischer, Daniel

daniel.fischer@nist.gov

Soft $\mathrm{x}$-ray absorption fine structure

$\mathrm{X}$-ray scattering

Surface science

Fuller, Edwin

edwin.fuller@nist.gov

Microstructural modeling and simulation

Microstructure effects on physical properties

Nanomechanical testing and properties

Toughening mechanisms

Gates, Richard

richard.gates@nist.gov

Tribo-chemistry of ceramics

Chemical analysis of ceramics

Nanometer film thickness measurements

Green, Martin

martin.green@nist.gov

Si microelectronic materials

Electronic ceramics

Combinatorial materials science

Hackley, Vincent

vince.hackley@nist.gov

Particle characterization

Particle dispersion and interface chemistry

Visco-elastic properties of suspensions

Harris, Joyce

joyce.harris@nist.gov

Data acquisition

Digitization and data entry

Hockey, Bernard

bernard.hockey@nist.gov

Transmission electron microscopy

Nanoindentation

Microstructural and defect characterization

Structure and composition of interfaces

Hsu, Stephen

stephen.hsu@nist.gov

Nanotribology

Nanolubrication

Magnetic hard disk interface

MEMs stiction

Huey, Bryan

bryan.huey@nist.gov

Atomic force microscopy

Domain stability

Ferroelectricity 
Kaiser, Debra

debra.kaiser@nist.gov

Electronic ceramics

Combinatorial material analysis

Karen, Vicky

vicky.karen@nist.gov

Crystallographic databases

Materials informatics

Lattice theory

Structural analysis

\section{Kelly, James}

james.kelly@nist.gov

Quantitative scanning electron microscopy

Image analysis

Microstructural analysis

Powder standards

Levin, Igor

igor.levin@nist.gov

Phase transitions in electronic ceramics

Crystal structure determination

Electron microscopy

Lufaso, Michael

michael.lufaso@nist.gov

Crystal structure-physical property correlation

Modeling and prediction of crystal structures

\section{Lum, Lin-Sien}

lin-sien.lum@nist.gov

Particle characterization

Instrumentation

\section{Munro, Ronald}

ronald.munro@nist.gov

Materials properties of advanced ceramics

Data evaluation and validation

Analysis of data relations

Paul, Albert

albert.paul@nist.gov

Laser physics

Residual stress measurement

\section{Quinn, George}

george.quinn@nist.gov

Mechanical property test standards

Standard reference materials

Fractography

\section{Robins, Lawrence}

lawrence.robins@nist.gov

Defect identification and distribution

Cathodoluminescence imaging/spectroscopy

Photoluminescence spectroscopy

Raman spectroscopy
Saylor, David

david.saylor@nist.gov

Microstructural characterization

Microstructural imaging

Grain boundary analysis

Schenck, Peter

peter.schenck@nist.gov

Emission and laser spectroscopy

Thin film deposition

Computer graphics and image analysis

Combinatorial materials analysis

Scotch, Adam

adam.scotch@nist.gov

Interfaces

Microstructural evolution

Sintering and grain growth

Electronic ceramics

Smith, Douglas

douglas.smith@nist.gov

Nanomechanics

Instrumented indentation

Surfaces forces

Adhesion

Sturrock, Charles

charles.sturrock@nist.gov

Materials informatics

Adaptive learning methods

Predictive data mining

Vanderah, Terrell

terrell.vanderah@nist.gov

Solid-state chemistry

Phase equilibria of microwave dielectrics

Vaudin, Mark

mark.vaudin@nist.gov

Electron microscopy

$\mathrm{X}$-ray and electron diffraction

Modeling of grain-boundary phenomena

Dielectric films

\section{Wagner, Richard}

richard.wagner@nist.gov

Nanoindentation

Modeling of phase transformation

Atomistic modeling of nanomechanics

White, Grady

grady.white@nist.gov

Residual stress measurements

Mechanical reliability

Raman spectroscopy 
Windover, Donald

donald.windover@nist.gov

High-resolution x-ray diffraction

X-ray reflectometry

Standard reference materials

Woicik, Joseph

joseph.woicik@nist.gov

UV photoemission

X-ray standing waves

Surface and interface science

Wong-Ng, Winnie

winnie.wong-ng@nist.gov

X-ray crystallography and reference patterns

Phase equilibria/crystal chemistry

High temperature superconductors

Yoder, Derek

derek.yoder@nist.gov

Soft x-ray absorption fine structure

X-ray scattering

Surface science

Ying, Charles

charles.ying@nist.gov

Atomic force microscopy

Nanofriction

Adhesion

\section{Research Associates}

\section{Armstrong, Nicholas}

University of Technology, Sydney, Australia

Bai, Mingwur

Tohoku University

Bartsch, Marian

Institute for Materials Research,

German Aerospace Center

Boukari, Hacene

University of Maryland

Bryson, Damian

Howard University

Cedeno, Christina

American Ceramic Society

Cha, Kum-hwan

Yousei University

Chae, Young-Hun

Kyungpook University
Coutts, Rachel

University of Maryland

Dapkunas, Stanley

Consultant

Dillingham, Jermey

University of Maryland

Evans, Howard

Smithsonian Institution

Fang, Hsu-Wei

University of Maryland

Farabaugh, Edward

American Ceramic Society

Haller, Wolfgang

Consultant

Harne, Mary

Consultant

Hastie, John

Consultant

Henins, Albert

Consultant

Hill, Kimberly

Consultant

Ilavsky, Jan

Purdue University

Jang, Mi-Hye

Yonsei University

Kang, Youn-Seon

Korea Advanced Institute of Science and Technology

Kim, Jang Yul

Hanyang University

Kim, Min-Soo

Korea Advanced Institute of Science and Technology

Kim, Yin Yong

Seoul National University

Kulkarni, Anand

State University of New York

Li, Xiang

Rensselaer Polytechnic Institute 
Liang, Yanang

Universided Oviedo, Spain

\section{Long, Gabrielle}

Argonne National Laboratory

Luong, Mario

University of Maryland

Mighell, Alan

Consultant

Ozmen, Yilmaz

Pamukkale University, Turkey

Pei, Patrick

Consultant

Peiris, Suhithi

Naval Research Laboratory

Piermarini, Gasper

Naval Surface Warfare Center

Prosandeev, Serguei

Rostov State University

Przedlacki, Marcin

Warshaw University of Technology

Purushotham, Kavuri

Swinbourn University

Rajagopalan, Arun

Rensselaer Polytechnic Institute

Rau, Christoph

Purdue University

Ritter, Joseph

Consultant
Roth, Robert

Viper Group

Seo, Chang-Eui

Korea Advanced Institute of Science and Technology

Suh, Julia

Consultant

Swanson, Nils

American Ceramic Society

Texter, John

National Science Foundation

Turchinskaya, Marina

Consultant

Wang, Xiao-lei

Tohoku University

Wang, Xuemin

American Ceramic Society

Wolfenstine, Jeffrey

U.S. Army Research Laboratory

Yang, Seung Ho

Korea Advanced Institute of Science and Technology

Yeager, Glenn

Trak Ceramics, Inc.

Ying, Tony

U.S. Mint of the Treasury Department

You, Jim

American Ceramic Society 



\section{Organizational Charts}

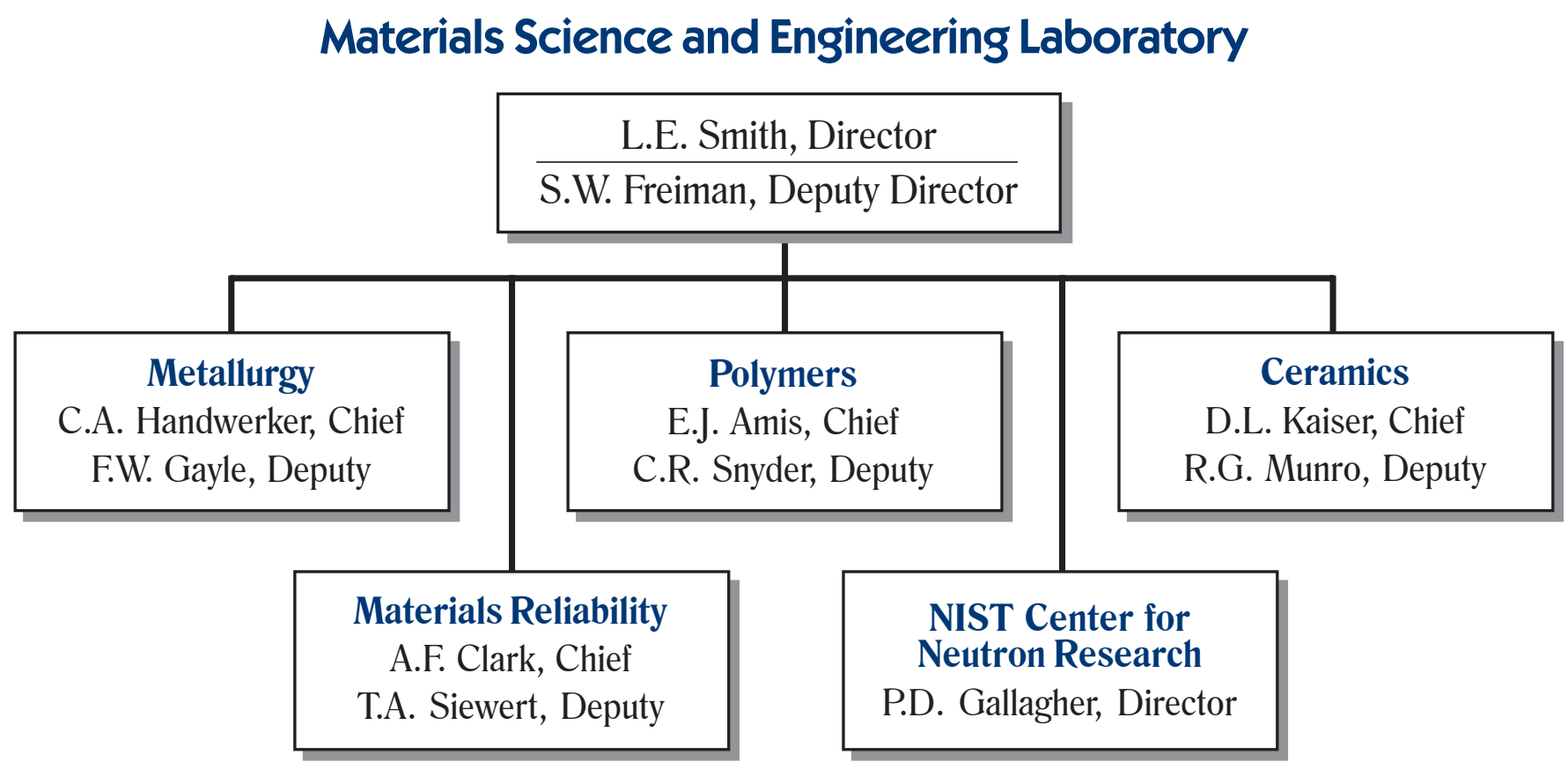

\section{National Institute of Standards and Technology}

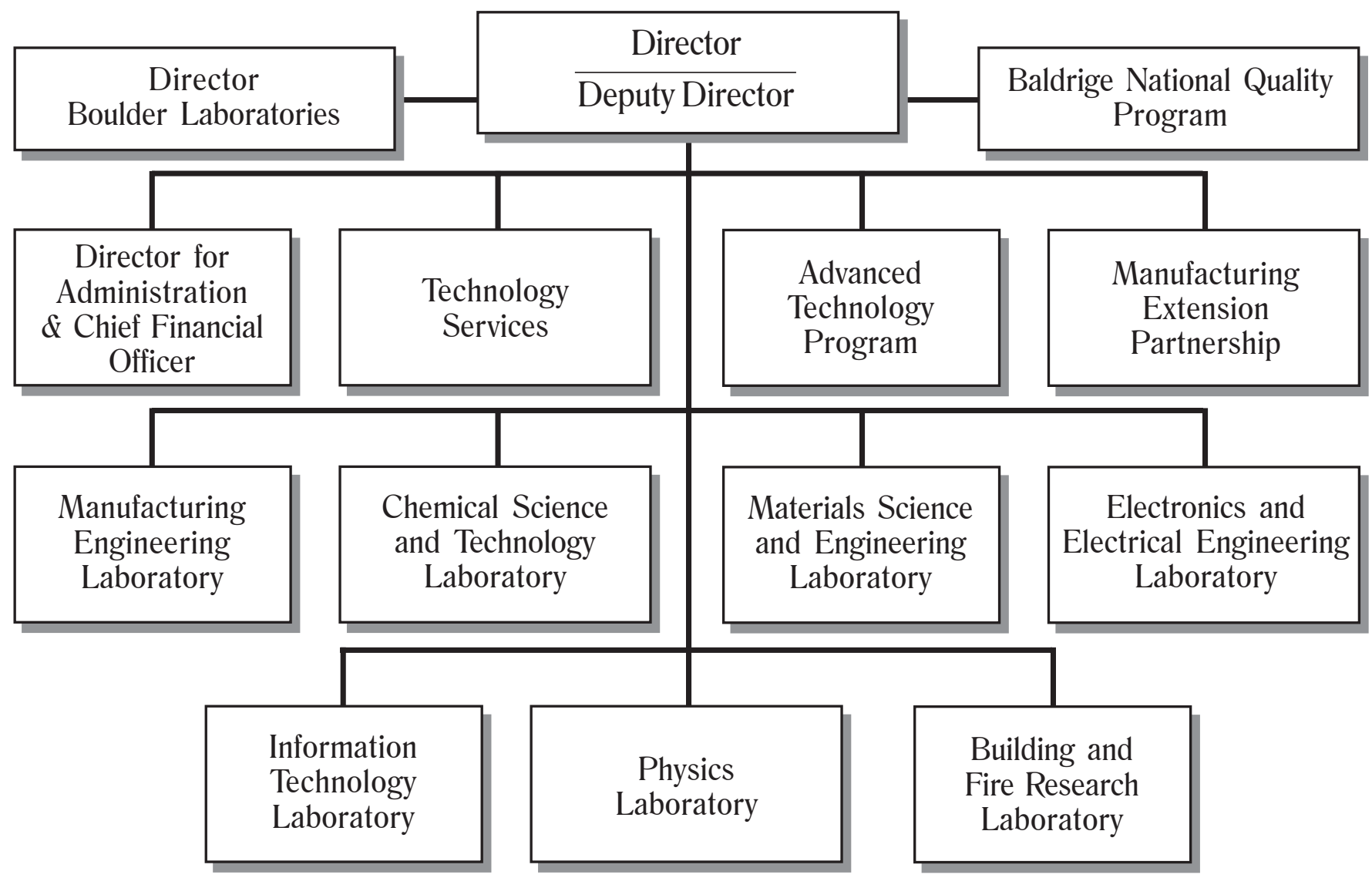






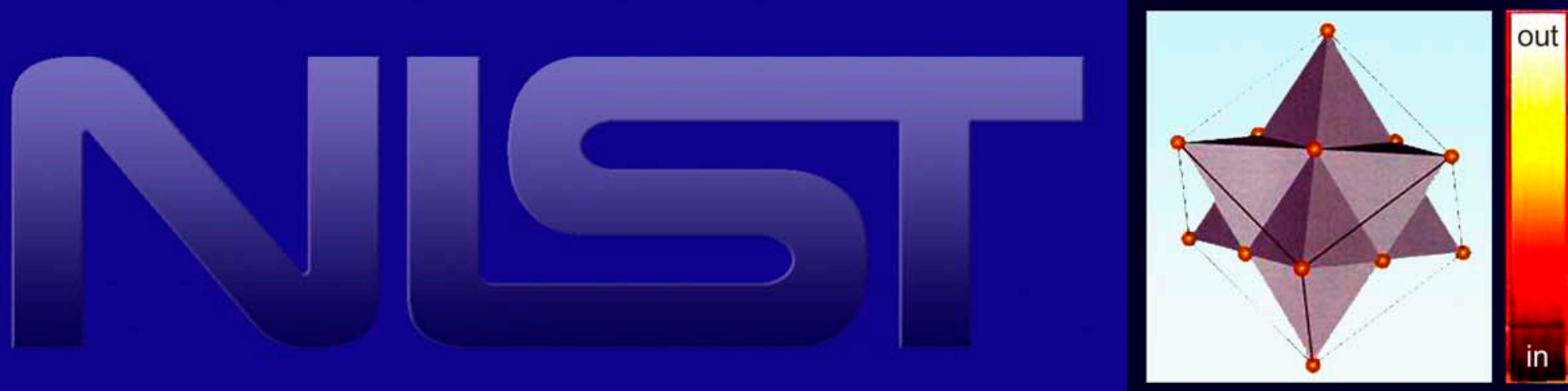